Geometry 85 Topology

Volume 7 (2003) 799-888

Published: 4 December 2003

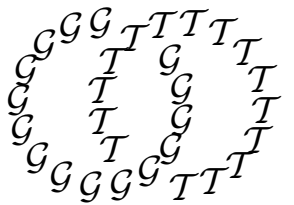

\title{
Compactness results in Symplectic Field Theory
}

\author{
F Bourgeois, Y Eliashberg, H Hofer \\ K Wysocki, E ZEHNDER
}

Universite Libre de Bruxelles, B-1050 Bruxelles, Belgium

Stanford University, Stanford, CA 94305-2125 USA

Courant Institute, New York, NY 10012, USA

The University of Melbourne, Parkville, VIC 3010, Australia ETH Zentrum, CH-8092 Zurich, Switzerland

\begin{abstract}
fbourgeo@ulb.ac.be, eliash@math.stanford.edu, hofer@cims.nyu.edu
K.Wysocki@ms.unimelb.edu.au, eduard.zehnder@math.ethz.ch
\end{abstract}

\begin{abstract}
This is one in a series of papers devoted to the foundations of Symplectic Field Theory sketched in [4]. We prove compactness results for moduli spaces of holomorphic curves arising in Symplectic Field Theory. The theorems generalize Gromov's compactness theorem in [8] as well as compactness theorems in Floer homology theory, [6, 7], and in contact geometry, $[9,19]$.
\end{abstract}

AMS Classification numbers Primary: 53D30

Secondary: 53D35, 53D05, 57R17

Keywords: Symplectic field theory, Gromov compactness, contact geometry, holomorphic curves

Proposed: Leonid Polterovich

Seconded: Robion Kirby, Simon Donaldson
Received: 19 August 2003 Accepted: 13 November 2003

(c) Geometry $\&$ Topology Publications 


\section{Introduction}

Starting with Gromov's work [8], pseudo-holomorphic curves (holomorphic curves in short) became a major tool in Hamiltonian systems, symplectic and contact geometries. Holomorphic curves are smooth maps

$$
F:(S, j) \rightarrow(W, J)
$$

from Riemann surfaces $(S, j)$ with a complex structure $j$ into almost complex manifolds $(W, J)$ with almost complex structure $J$ (ie, $J^{2}=-$ id) having the property that their linearized maps $d F$ are complex linear at every point. Hence $F$ satisfies the elliptic system of first order partial differential equations

$$
d F \circ j=J(F) \circ d F .
$$

Our aim is to investigate the compactness properties of the set of holomorphic curves varying not only the maps but also their domains consisting of punctured and nodal Riemann surfaces, as well as (in certain cases) their targets. Another major difference from Gromov's set-up is that the target manifolds of the holomorphic curves under consideration are not necessarily compact, and not necessarily having finite geometry at infinity. The target manifolds are almost complex manifolds with cylindrical ends and, in particular, cylindrical almost complex manifolds. We also consider the effect of a deformation of the almost complex structure on the target manifold leading to its splitting into manifolds with cylindrical ends. This deformation is an analogue of the "stretching of the neck" operation, popular in gauge theory. Our analysis introduces the new concepts of stable holomorphic buildings and makes use of the well known Deligne-Mumford compactification of Riemann surfaces. The main results are formulated and proven in Section 10. We will see that the moduli spaces of stable buildings, whose holomorphic maps have uniformly bounded energies and whose domains have a fixed arithmetic genus and a fixed number of marked points, are compact metric spaces. The compactness results for holomorphic curves proved in this paper cover a variety of applications, from the original Gromov compactness theorem for holomorphic curves [8], to Floer homology theory [6, 7], and to Symplectic Field Theory [4]. In fact, all compactness results for holomorphic curves without boundary known to us, including the compactness theorems in [21, 22], [23] and [24], follow from the theorems we shall prove here. Gromov's compactness theorem for closed holomorphic curves asserts compactness under the condition of the boundedness of the area. The holomorphic curves we consider are proper holomorphic maps of Riemann surfaces with punctures into non-compact manifolds, thus they usually have infinite area. The bound on the area as the condition for compactness is replaced here 
by the bound on another quantity, called energy. In applications of Gromov's theorem it is crucial to get an a priori bound on the area. The only known case in which the a priori estimates can be obtained is the case in which the almost complex structure is calibrated or tamed by a symplectic form. For this reason we decided to include the appropriate taming conditions in the statement of the theorems. This brings certain simplifications in the proofs and still allows to cover all currently known applications.

Outline of the paper We begin in Sections 2 and 3 with basics about cylindrical almost complex manifolds and almost complex manifolds with cylindrical ends. We also describe the process of splitting of a complex manifolds along a real hypersurface and appropriate symplectic taming conditions which will enable us to prove the compactness results. In Section 4 we recall standard facts about hyperbolic geometry of Riemann surfaces and define the DeligneMumford compactification of the space of punctured Riemann surfaces, and its slightly bigger decorated version. In Sections 5 and 6 we define important notions of contact and symplectic energy and discuss the asymptotic behavior and other important analytic facts about holomorphic curves satisfying appropriate energy bounds. Sections 7-8 are devoted to the description of the compactification of the moduli spaces of holomorphic curves in cylindrical manifolds, manifolds with cylindrical ends and in the families of almost complex manifolds which appear in the process of splitting. Section 10 is the central section of the paper, where we prove our main compactness results. In Section 11 we formulate some other related compactness theorems which can be proven using analytic techniques developed in the paper. Finally, in Appendix A we prove the necessary asymptotic convergence estimates, and in Appendix B describe metric structures on the compactified moduli spaces of holomorphic curves.

Acknowledgements Parts of this paper were written while some of the authors visited the Institute for Advanced Study at Princeton, Courant Institute for Mathematical Sciences at NYU, New York and Forschungsinstitut für Mathematik at the ETH, Zurich. The authors thank all these institutions for the hospitality. The authors are also grateful to the American Institute of Mathematics at Palo Alto for organizing very stimulating workshops and a special program on contact geometry. Finally, the authors want to thank the referee for his critical remarks.

F Bourgeois is partially supported by FNRS, the CMI Liftoff program and EDGE; Y Eliashberg is partially supported by an NSF grant and the Oswald Veblen Foundation; H Hofer is partially supported by an NSF grant, a Clay 
scholarship and the Wolfensohn Foundation; K Wysocki is partially supported by an Australian Research Council grant; E Zehnder is partially supported by the TH-project.

\section{Cylindrical almost complex manifolds}

\subsection{Cylindrical almost complex structures}

An almost complex structure $J$ on $\mathbb{R} \times V$ is called cylindrical if it is invariant under translations

$$
(t, x) \mapsto(t+c, x), t, c \in \mathbb{R}, x \in V,
$$

and the vector field $\mathbf{R}=J \frac{\partial}{\partial t}$ is horizontal, ie, tangent to levels $t \times V, t \in$ $\mathbb{R}$. Clearly, any cylindrical structure on $\mathbb{R} \times V$ is determined by the CRstructure $\left(\xi=J T V \cap T V, J_{\xi}=\left.J\right|_{\xi}\right)$ and the restriction of the vector field $\mathbf{R}$ to $V=\{0\} \times V$, which also will be denoted by $\mathbf{R}$. Furthermore, the distribution $\xi$ and the vector field $\mathbf{R}$ uniquely determine a 1 -form $\lambda=\lambda_{J}$ on $V$ satisfying $\lambda(\mathbf{R}) \equiv 1$ and $\left.\lambda\right|_{\xi}=0$. The cylindrical structure $J$ is called symmetric if $\lambda$ is preserved by the flow of $\mathbf{R}$, ie, if the Lie derivative $L_{\mathbf{R}} \lambda$ vanishes. This is equivalent to the condition $\mathbf{R}\lrcorner d \lambda=0$ in view of the Cartan formula $\left.L_{\mathbf{R}} \lambda=\mathbf{R}\right\lrcorner d \lambda+d(\lambda(\mathbf{R}))$. It is important to point out that the flow of $\mathbf{R}$ is not required to preserve $J$ itself. Here are three important examples of symmetric cylindrical almost complex structures.

Example 2.1 Suppose that $\lambda$ is a contact form and $\mathbf{R}$ its Reeb vector field. Let us recall that this means that $\lambda \wedge(d \lambda)^{n-1}$, where $\operatorname{dim} V=2 n-1$, is a volume form on $V, \mathbf{R}$ generates the kernel of $d \lambda$ and is normalized by the condition $\lambda(\mathbf{R})=1$. Then $L_{\mathbf{R}} \lambda=0$, and hence, any $J$ with $\lambda=\lambda_{J}$ is automatically symmetric. We refer to this example as to the contact case. It is important to observe that in the contact case the levels $c \times V, c \in \mathbb{R}$, are strictly pseudo-convex being co-oriented as boundaries of the domains $(-\infty, c] \times V$.

Example 2.2 Let $\pi: V \rightarrow M$ be a principal $S^{1}$-bundle over a closed manifold $M$ and $\mathbf{R}$ the vector field which is the infinitesimal generator of the $S^{1}$-action. Let $\lambda$ be an $S^{1}$-connection form on $V$ such that $\lambda(\mathbf{R}) \equiv 1$. Then we have $L_{\mathbf{R}} \lambda=0$ and hence any cylindrical almost complex structure $J$ with $\lambda_{J}=\lambda$ is symmetric. 
Example 2.3 Suppose that the 1 -form $\lambda$ is closed. The equality $d \lambda=0$ trivially implies $\mathbf{R}\lrcorner d \lambda=0$, and hence the corresponding $J$ is symmetric. This means, in particular, that the distribution $\xi$ is integrable and thus the CR-structure $\left(\xi, J_{\xi}\right)$ is Levi-flat.

The tangent bundle $T(\mathbb{R} \times V)$ of a cylindrical manifold canonically splits as a direct sum of two complex subbundles, namely into the bundle $\xi$ and the trivial 1 -dimensional complex bundle generated by $\frac{\partial}{\partial t}$. In this paper the manifold $V$ is mostly assumed to be closed, though in the last Section 11.1 we will discuss some results for non-compact $V$.

\subsection{Taming conditions}

It was first pointed out by M. Gromov (see [8]) that though the local theory of holomorphic curves in an almost complex manifold is as rich as in the integrable case, the meaningful global theory does not exist unless there is a symplectic form which tames the almost complex structure. Let us recall that a linear complex structure $J$ on a vector space $E$ is called tamed by a symplectic form $\omega$ if the form $\omega$ is positive on complex lines. If, in addition, one adds the calibrating condition that $\omega$ is $J$-invariant, then $J$ is said to be compatible with $\omega$. In the latter case

$$
\omega(X, J Y)-i \omega(X, Y), \quad X, Y \in E,
$$

is a Hermitian metric on $E$. Let $(\mathbb{R} \times V, J)$ be a cylindrical almost complex manifold and let $\left(\xi, J_{\xi}\right)$ and $\mathbf{R} \in T V$ be the $\mathrm{CR}$-structure and the vector field which determine $J$, and $\lambda=\lambda_{J}$ the corresponding 1-form. Given a maximal rank closed 2-form $\omega$ on $V$ we say, to avoid an overused word "compatible", that $J$ is adjusted to $\omega$ if $\left.\omega\right|_{\xi}$ is compatible with $J_{\xi}$ and, in addition,

$$
\left.L_{\mathbf{R}} \omega=\mathbf{R}\right\lrcorner \omega=0 .
$$

The latter condition means that the vector field $\mathbf{R}$ is Hamiltonian with respect to $\omega$. Our prime interest in this paper are symmetric cylindrical almost complex structures adjusted to a certain closed 2-form. Let us review the adjustment conditions in the Examples 2.1-2.3. In the contact case from Example $2.1 J$ is adjusted to $\omega=d \lambda$. When referring to the contact case we will always assume that $d \lambda$ is chosen as the taming form. Suppose that $\pi: V \rightarrow M, \lambda$ and $R$ are as in Example 2.2. Suppose that the manifold $M$ is endowed with a compatible symplectic form $\underline{\omega}$ and an almost complex structure $\underline{J}$. Set $\omega=\pi^{*} \underline{\omega}$ and lift $\underline{J}$ to $J_{\xi}$ on $\xi$ via the projection $\pi$. Then the symmetric cylindrical almost complex 
structure $J$ on $\mathbb{R} \times V$ determined by the vector field $\mathbf{R}$ and the CR-structure $\left(\xi, J_{\xi}\right)$ is adjusted to the form $\omega$. The adjustment condition in Example 2.3 requires the existence of a closed 2 -form $\omega$ on $V$ whose restriction to the leaves of the foliation $\xi$ is symplectic and is compatible with $J_{\xi}$. An important special case of this construction, which appears in the Floer homology theory, is the case in which the form $\lambda$ has an integral cohomology class and hence the manifold $V$ fibers over the circle $S^{1}$ with symplectic fibers. If $(M, \eta)$ is the fiber of this fibration then $V$ can be viewed as the mapping torus

$$
V=[0,1] \times M /\{(0, x) \sim(1, f(x)), x \in M\}
$$

of a symplectomorphism $f$ of a symplectic manifold $(M, \eta)$.

\subsection{Dynamics of the vector field $R$}

Suppose that a symmetric cylindrical almost complex structure $J$ is adjusted to a closed form $\omega$. This implies that the vector field $\mathbf{R}$ is Hamiltonian: its flow preserves the form $\omega$. Let us denote by $\mathcal{P}=\mathcal{P}_{J}$ the set of periodic trajectories, counting their multiples, of the vector field $\mathbf{R}$ restricted to $V=\{0\} \times V$. Generically, $\mathcal{P}$ consists of only countably many periodic trajectories. Moreover, these trajectories can be assumed to be non-degenerate in the sense that the linearized Poincaré return map $A_{\gamma}$ along any closed trajectory $\gamma$, including multiples, has no eigenvalues equal to 1 . We will refer to this generic case as to the Morse case. We will be also dealing in this paper with a somewhat degenerate, so-called Morse-Bott case. Notice that any smooth family of periodic trajectories from $\mathcal{P}$ has the same period. Indeed, suppose we are given a map $\Phi: S^{1} \times[0,1] \rightarrow V$ satisfying $\gamma_{\tau}=\left.\Phi\right|_{S^{1} \times \tau} \in \mathcal{P}$ for all $\tau \in[0,1]$. Let us denote by $T_{\tau}$ the period of $\gamma_{\tau}$. Then we have

$$
T_{1}-T_{0}=\int_{\gamma_{1}} \lambda-\int_{\gamma_{0}} \lambda=\int_{S^{1} \times[0,1]} \Phi^{*} d \lambda=0,
$$

in view of $\mathbf{R}\lrcorner d \lambda=0$ so that $\Phi^{*} d \lambda$ vanishes on $S^{1} \times[0,1]$. We say that a symmetric cylindrical almost complex structure $J$ is of the Morse-Bott type if, for every $T>0$ the subset $N_{T} \subset V$ formed by the closed trajectories from $\mathcal{P}$ of period $T$ is a smooth closed submanifold of $V$, such that the rank of $\left.\omega\right|_{N_{T}}$ is locally constant and $T_{p} N_{T}=\operatorname{ker}\left(d\left(\varphi_{T}\right)-I\right)_{p}$, where $\varphi_{t}: V \rightarrow V$ is the flow generated by the vector field $\mathbf{R}$.

In this paper we will only consider symmetric cylindrical almost complex structures $J$ for which either the Morse, or the Morse-Bott condition is satisfied. 
Given two homotopic periodic orbits $\gamma, \gamma^{\prime} \in \mathcal{P}$ and a homotopy $\Phi: S^{1} \times[0,1] \rightarrow$ $V$ connecting them, we define their relative $\omega$-action by the formula ${ }^{1}$

$$
\Delta S_{\omega}\left(\gamma, \gamma^{\prime} ; \Phi\right)=\int_{S^{1} \times[0,1]} \Phi^{*} \omega .
$$

If the taming form $\omega$ is exact, ie, $\omega=d \theta$ for a one-form $\theta$ on $V$, then

$$
\Delta S_{\omega}\left(\gamma, \gamma^{\prime} ; \Phi\right)=\int_{\gamma} \theta-\int_{\gamma^{\prime}} \theta,
$$

so that the relative action is independent of the homotopy $\Phi$.

We will also introduce the $\lambda$-action, or simply the action of a periodic $\mathbf{R}$-orbit $\gamma$ by

$$
S(\gamma)=\int_{\gamma} \lambda
$$

Thus, in the contact case in which $\omega=d \lambda$,

$$
\Delta S_{\omega}\left(\gamma, \gamma^{\prime} ; \Phi\right)=S(\gamma)-S\left(\gamma^{\prime}\right)
$$

\section{Almost complex manifolds with cylindrical ends}

\subsection{Remark about gluing two manifolds along their boundary}

We consider two manifolds $W$ and $W^{\prime}$ with boundaries and let $V$ and $V^{\prime}$ be their boundary components. Given a diffeomorphism $f: V \rightarrow V^{\prime}$, the manifold

$$
\widetilde{W}=W \bigcup_{V \stackrel{f}{\sim} V^{\prime}} W^{\prime}
$$

glued along $V$ and $V^{\prime}$ is defined as a piecewise smooth manifold. If $W$ and $W^{\prime}$ are oriented, and $f: V \rightarrow V^{\prime}$ reverses the orientation then $\widetilde{W}$ inherits the orientation. However, to define a smooth structure on $\widetilde{W}$ one needs to make some additional choices (eg, one has to choose embeddings $I:(-\varepsilon, 0] \times V \rightarrow W$ and $F:\left[0, \varepsilon^{\prime}\right) \times V \rightarrow W^{\prime}$ such that $\left.I\right|_{0 \times V}$ is the inclusion $V \hookrightarrow W$ and $\left.F\right|_{0 \times V}$ is the composition $\left.V \stackrel{f}{\rightarrow} V^{\prime} \hookrightarrow W^{\prime}\right)$. Suppose that the manifolds $W$ and $W^{\prime}$ are

\footnotetext{
${ }^{1}$ Sometimes, when it will be explicitly said so, we consider the relative $\omega$-action between the $\mathbf{R}-$ orbits when one, or both of the orbits come with the opposite orientation.
} 
endowed with almost complex structures $J$ and $J^{\prime}$. Then the tangent bundles $T V$ and $T V^{\prime}$ carry $C R$-structures, ie, complex subbundles

$$
\xi=J T W \cap T W \quad \text { and } \quad \xi^{\prime}=J T W^{\prime} \cap T W^{\prime} .
$$

Then, in order to define an almost complex structure on $\widetilde{W}=W \bigcup_{V^{\prime} \stackrel{f}{\sim}(V)} W^{\prime}$ the orientation reversing diffeomorphism $f$ must preserve these structures. In other words, we should have $d f(\xi)=\xi^{\prime}$ and $d f:(\xi, J) \rightarrow\left(\xi^{\prime}, J^{\prime}\right)$ should be a homomorphism of complex bundles. In this case $d f$ canonically extends to a complex bundle homomorphism

$$
\widetilde{d f}:\left(\left.T W\right|_{V}, J\right) \rightarrow\left(\left.T W^{\prime}\right|_{V^{\prime}}, J^{\prime}\right),
$$

and thus allows us to define a $C^{1}$-smooth structure on $\widetilde{W}$ and a continuous almost complex structure $\widetilde{J}$ on $\widetilde{W}$. To define a $C^{\infty}$-smooth structure $\widetilde{J}$ on $\widetilde{W}$ we may need not only to choose some additional data, as in the case of a smooth structure, but also to perturb either $J$ or $J^{\prime}$ near $V$. A particular choice of the perturbation will usually be irrelevant for us, and thus will not be specified.

Suppose now that $(W, \Omega)$ and $\left(W^{\prime}, \Omega^{\prime}\right)$ are symplectic manifolds with boundaries $V$ and $V^{\prime}$ and introduce $\omega=\left.\Omega\right|_{V}, \omega^{\prime}=\left.\Omega^{\prime}\right|_{V^{\prime}}$. Suppose that there exists a diffeomorphism $f: V \rightarrow V^{\prime}$ which reverses the orientations induced on $V$ and $V^{\prime}$ by the symplectic orientations of $W$ and $W^{\prime}$ and which satisfies $f^{*} \omega^{\prime}=\omega$. Notice that for any 1 -form $\lambda$ on $V$ which does not vanish on the (1-dimensional) kernel of the form $\omega$ we can form for a sufficiently small $\varepsilon>0$ a symplectic manifold $((-\varepsilon, \varepsilon) \times V, \widetilde{\omega}+d(t \lambda))$, where $t \in(-\varepsilon, \varepsilon)$ and $\widetilde{\omega}$ is the pull-back of $\omega$ under the projection $(-\varepsilon, \varepsilon) \times V \rightarrow V$. According to a version of Darboux' theorem any symplectic manifold containing a hypersurface $(V, \omega)$, is symplectomorphic near $V$ to $((-\varepsilon, \varepsilon) \times V, \widetilde{\omega}+d(t \lambda))$ via a symplectomorphism fixed on $V$. In particular, the identity map $V \rightarrow 0 \times V$ extends to a symplectomorphism of a neighborhood of $V$ in $W$ onto the lower-half $(-\varepsilon, 0] \times V)$, while the map $f^{-1}: V^{\prime} \rightarrow 0 \times V$ extends to a symplectomorphism of a neighborhood of $V^{\prime}$ in $W^{\prime}$ onto the upper half $[0, \varepsilon) \times V$. This allows us to glue $W$ and $W^{\prime}$ into a smooth symplectic manifold $W \underset{f}{\cup} W^{\prime}$. The symplectic structure on this manifold is independent of extra choices up to symplectomorphisms which are the identity maps on $V=V^{\prime}$ and also outside of a neighborhood of this hypersurface. Hence these extra choices will not be usually specified. 


\subsection{Attaching a cylindrical end}

Let $(\bar{W}, J)$ be a compact smooth manifold with boundary and let $J^{\prime}$ a cylindrical almost complex structure on $\mathbb{R} \times V$ where $V=\partial \bar{W}$. If $J$ and $J^{\prime}$ induce on $V$ the same CR-structure then, depending on whether the orientation of $V$ determined by $\left.J^{\prime}\right|_{\xi^{\prime}}$ and $\mathbf{R}$, is opposite or coincides with the orientation of the boundary of $\bar{W}$ we can, as it is described in Section 3.1 above, attach to $(\bar{W}, J)$ the positive cylindrical end $\left(E_{+}=[0, \infty) \times V,\left.J^{\prime}\right|_{E_{+}}\right)$, or the negative cylindrical end $\left(E_{-}=(-\infty, 0] \times V,\left.J^{\prime}\right|_{E_{-}}\right)$, ie, we consider the manifold

$$
W=\bar{W} \underset{V=0 \times V}{\cup} E_{ \pm}
$$

with the induced complex structure, still denoted by $J$. Alternatively, we say that an almost complex manifold $\left(X, J_{X}\right)$ has a cylindrical end (or ends if $X$ is disconnected at infinity) if it is biholomorphically equivalent to a manifold of the form (2). To get a concrete model of this kind (for a positive end), let us choose a tubular neighborhood $U=[-1,0] \times V$ of $V=\partial \bar{W}$ in $\bar{W}$. Let $g^{\delta}:[-\delta, \infty) \rightarrow[-\delta, 0)$, with $0<\delta<1$, be a monotone and (non-strictly) concave function which coincides with

$$
t \mapsto-\frac{\delta}{2} e^{-t}
$$

for $t \in[0, \infty)$ and which is the identity map near $-\delta$. We define a family of diffeomorphisms $G^{\delta}: W \rightarrow \stackrel{\circ}{W}=\operatorname{Int} \bar{W}$ by means of the formula

$$
G^{\delta}(w)= \begin{cases}\left(g^{\delta}(t), x\right), & w=(t, x) \in U^{\delta} \cup E_{+} \\ w, & w \in W \backslash\left(U^{\delta} \cup E_{+}\right),\end{cases}
$$

where $U^{\delta}=[-\delta, 0] \times V \subset U$. The push-forward $\left(G^{\delta}\right)^{*} J$ will be denoted by $J^{\delta}$. Thus $\left(\stackrel{\circ}{W}, J^{\delta}\right)$ can be viewed as another model of an almost complex manifold with cylindrical end. We say that $\left(X, J_{X}\right)$ has asymptotically cylindrical positive resp. negative end if there exists a diffeomorphism

$$
f: W=\bar{W} \underset{V=0 \times V}{\cup} E_{ \pm} \rightarrow X
$$

such that the families of mappings $f^{s}: E_{ \pm} \rightarrow X$, for $s \geq 0$, defined on the ends by the formulae $f^{s}(t, x)=f(t \pm s, x)$ have the following properties,

- $J^{s}:=\left(f^{s}\right)^{*} J_{X} \longrightarrow J$ in $C_{\mathrm{loc}}^{\infty}$.

- $J^{s}\left(\frac{\partial}{\partial t}\right)=\mathbf{R}$ for all $s \geq 0$.

We say that $W$ has a symmetric cylindrical end if the almost complex structure $\left.J\right|_{E_{ \pm}}$is symmetric, ie, $L_{\mathbf{R}} \lambda=0$ where $\mathbf{R}$ and $\lambda$ are the vector field and the 
1-form on $V$ introduced in the definition of a cylindrical almost complex structure. The notion of an almost complex manifold with symmetric asymptotically cylindrical end has an obvious meaning.

Examples 3.1 (1) $\mathbb{C}^{n}$ has a symmetric (and even contact type) cylindrical end.

(2) For any complex manifold $W$ the punctured manifold $W \backslash\{p\}$ has a cylindrical symmetric end.

(3) More generally, let $(X, J)$ be an almost complex manifold, and $Y \subset X$ an almost complex submanifold of any real codimension $2 k$. Then the manifold $\left(X \backslash Y,\left.J\right|_{X \backslash Y}\right)$ has a symmetric asymptotically cylindrical end corresponding to the cylindrical manifold $E=\mathbb{R} \times V$, where $V$ is the sphere bundle over $Y$ associated with the complex normal bundle to $Y$ in $X$, and the vector field $\mathbf{R}$ is tangent to the fibers. In particular, there is another almost complex structure $J^{\prime}$ on $X$ which is $C^{1}$-close to $J$ and coincides with $J$ on $\left.T(X)\right|_{Y}$ such that the almost complex manifold $\left(X \backslash Y,\left.J^{\prime}\right|_{X \backslash Y}\right)$ has a symmetric cylindrical end.

(4) Let $(S \backslash Z, j)$ be a closed Riemann surface with punctures, and $(M, J)$ be any almost complex manifold. Then $((S \backslash Z) \times M, j \oplus J)$ has cylindrical ends.

\subsection{Taming conditions for almost complex manifolds with cylin- drical ends}

Let $\left(W=\bar{W} \cup \cup_{V} E, J\right)$ be an almost complex manifold with a cylindrical end $E \subset \mathbb{R} \times V$ where $V=\partial \bar{W}$. Let $\mathbf{R}$ be the associated vector field tangent to $V$, and let $\left(\xi, J_{\xi}\right)$ be the CR-structure, and $\lambda$ the 1 -form on $V$ associated with the cylindrical structure $\left.J\right|_{E}$. Suppose that the manifold $\bar{W}$ admits a symplectic form $\omega$. We say that the almost complex structure $J$ is adjusted to $\omega$ if

- the symplectic form $\omega$ is compatible with $\left.J\right|_{\bar{W}}$,

- $\left.J\right|_{E}$ is adjusted to $\omega$ is the sense of the definition in Section 2.2.

Notice that the distinction between positive and negative ends depends on the 1 -form $\lambda$. In the contact case, ie, when the 1 -form $\lambda$ associated with a cylindrical end $E$ is a contact form and $\omega=d \lambda$, this sign is beyond our control; the boundary component $V_{0}$ is positive iff there exists an outgoing vector field $X$ transversal to $V_{0}$ which dilates the symplectic form $\omega$, ie, $L_{X} \omega=\omega$. On 
the other hand, in other cases (see, for instance Examples 2.2 and 2.3) the sign of $\lambda$, and hence the sign of the corresponding end $E$ can be changed at our will. We will use the notation $E_{-}$respectively $E_{+}$for the union of the negative ends respectively the positive ends. Similarly, the set $\mathcal{P}$ of periodic orbits of the vector field $\mathbf{R}$ also splits into the disjoint sets $\mathcal{P}_{-}$and $\mathcal{P}_{+}$of orbits on $V_{-}$ and $V_{+}$. Consider now an almost complex structure $J$ on $W=\bar{W} \cup_{V} E$ with an asymptotically cylindrical end. The structure $J$ is called adjusted to $\omega$ if

- $\omega$ is compatible with $\left.J\right|_{\bar{W}}$,

- $\left.\omega\right|_{\xi}$ is compatible with $\left.J_{t}\right|_{\xi}$ for each $t \geq 0$ (or $t \leq 0$ ) where $J_{t}$ is the pull-back of $J$ under the inclusion map $V=V \times t \hookrightarrow E$.

Note that in the situation of the last definition, the 2 -form $\omega$ can always be extended to $W=\bar{W} \cup_{V} E$ as a symplectic form $\widetilde{\omega}$ taming $J$.

\subsection{Splitting}

The following splitting construction is an important source of manifolds with cylindrical ends. Let $W$ be a closed almost complex manifold, or a manifold with cylindrical ends, and $V \subset W$ a co-orientable compact real hypersurface. Let $\xi=T V \cap J T V$ be the CR-structure induced on $V$, ie, the distribution of maximal complex tangent subspaces of $T V$. Let us cut $W$ open along $V$. The boundary of the newly created manifold $\stackrel{\circ}{W}$ (which is disconnected if $V$ divides $W$ ) consists of two copies $V^{\prime}, V^{\prime \prime}$ of $V$. Choose any vector field $\mathbf{R} \in T V$ transverse to $\xi$ and attach to $\stackrel{\circ}{W}$ the ends $E_{+}=[0, \infty) \times V$ and $E_{-}=(-\infty, 0] \times V$ with the unique cylindrical almost complex structure determined by the CR-structure $\left(\xi, J_{\xi}\right)$ and the vector field $\mathbf{R}$. As it was pointed out in Section 3.1 the resulting manifold

$$
\widetilde{W}=E_{-} \underset{V \times 0=V^{\prime}}{\cup} \stackrel{\circ}{W} \underset{V^{\prime \prime}=V \times 0}{\cup} E_{+} .
$$

gets a canonical $C^{1}$-smooth structure and a continuous almost complex structure $\widetilde{J}$. In order to make $\widetilde{J}$ smooth in the $C^{\infty}$ sense we may need to perturb $J$ on $\stackrel{\circ}{W}$ near $V$. A specific choice of the perturbation will be irrelevant for our purposes, and thus not specified. The manifold $(\widetilde{W}, \widetilde{J})$ has cylindrical ends and is diffeomorphic to $W \backslash V$. Note that the above splitting construction can be viewed as the result of a "stretching of the neck". Indeed one can consider a family of manifolds

$$
W^{\tau}=\stackrel{\circ}{W} \underset{V^{\prime \prime}=(-\tau) \times V, \tau \times V=V^{\prime}}{\cup}[-\tau, \tau] \times V, \tau \in[0, \infty),
$$


with the almost complex structure $J^{\tau}$ on the insert $[-\tau, \tau] \times V$ which is uniquely determined by the same translational invariance condition. Then we have

$$
\left(W^{\tau}, J^{\tau}\right) \underset{\tau \rightarrow \infty}{\longrightarrow}(\widetilde{W}, \widetilde{J}) .
$$

Suppose that $\omega$ is a symplectic form on $W$ compatible with $J$, the vector

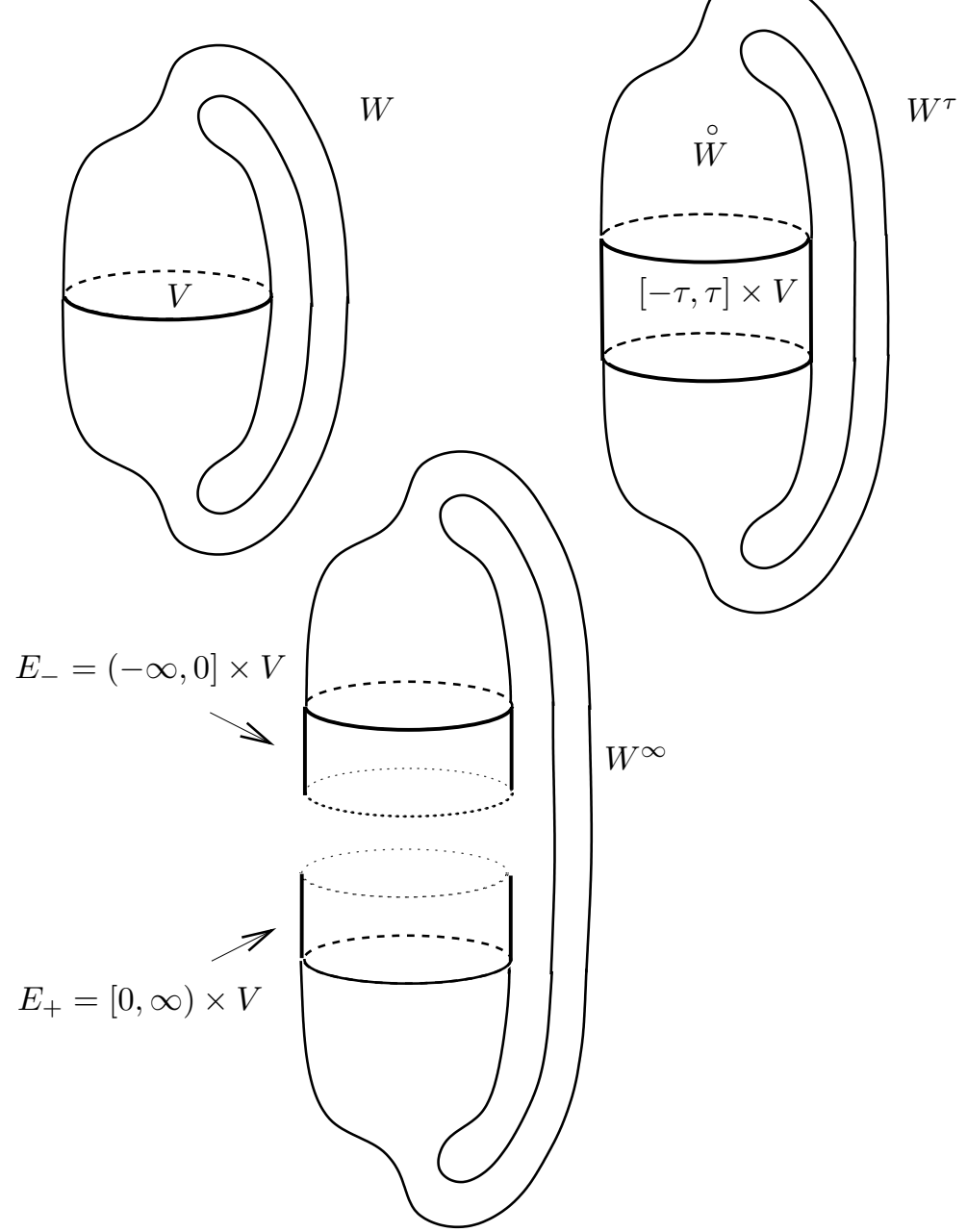

Figure 1: Splitting

field $\mathbf{R}$ on the hypersurface $V$ generates the kernel of $\left.\omega\right|_{V}$, and the cylindrical structure on $\mathbb{R} \times V$ defined by $J$ and $\mathbf{R}$ is symmetric. We say that in this case the splitting data are adjusted to the symplectic form $\omega$. This is, in particular, the case when $V$ is a contact type hypersurface in $W$, ie, a hypersurface which 
admits in its neighborhood a transversal conformally symplectic vector field. In the adjusted case the splitting construction gives a manifold with symmmetric cylindrical ends which is adjusted to $\omega$. Note that the hypersurface $V$ is not assumed to divide $W$. On the other hand, $V$ is allowed to be disconnected and to split $W$ into several connected components. Finally, the above splitting construction immediately generalizes to the case when the manifold $W$ itself has cylindrical ends.

\section{Deligne-Mumford compactness revisited}

\subsection{Smooth stable Riemann surfaces}

Let $\mathbf{S}=(S, j, M)$ be a compact connected Riemann surface(without boundary) with a set $M$ of numbered disjoint marked points. Two Riemann surfaces $\mathbf{S}=(S, j, M)$ and $\mathbf{S}^{\prime}=\left(S^{\prime}, j^{\prime}, M^{\prime}\right)$ are called equivalent if there exists a diffeomorphism $\varphi: S \rightarrow S^{\prime}$ such that $\varphi_{*} j=j^{\prime}$ and $\varphi(M)=M^{\prime}$ where we assume that $\varphi$ preserves the ordering of the sets $M$ and $M^{\prime}$. Let $\mu$ be the cardinality of $M$, and $g$ the genus of $S$. The surface is called stable if

$$
2 g+\mu \geq 3 .
$$

The stability condition is equivalent to the requirement that the group of conformal automorphisms of $\mathbf{S}$, ie, biholomorphic maps preserving the marked points, is finite. The pair $(g, \mu)$ is called the signature of the Riemann surface $\mathbf{S}$. Given a stable surface $\mathbf{S}=(S, j, M)$, the Uniformization Theorem asserts the existence of a unique complete hyperbolic metric of constant curvature -1 of finite volume, in the given conformal class $j$ on $\dot{S}=S \backslash M$. We will denote this metric by $h^{\mathbf{S}}=h^{j, M}$. Each puncture corresponds to a cusp of the hyperbolic metric $h^{j, M}$. In what follows we will always assume for a given stable Riemann surface $(S, j, M)$ that the punctured surface $\dot{S}=S \backslash M$ is endowed with the uniformizing hyperbolic metric $h^{j, M}$. Thus the moduli space $\mathcal{M}_{g, \mu}$ of the stable Riemann surfaces of signature $(g, \mu)$ can be viewed equivalently as the moduli space of (equivalence classes of) hyperbolic metrics of finite volume and of constant curvature -1 on the fixed surface $\dot{S}=S \backslash M$. 


\subsection{Thick-thin decomposition}

Fix an $\varepsilon>0$. Given a stable Riemann surface $\mathbf{S}=(S, j, M)$ we denote by $\operatorname{Thick}_{\varepsilon}(\mathbf{S})$ and $\operatorname{Thin}_{\varepsilon}(\mathbf{S})$ its $\varepsilon$-thick and $\varepsilon$-thin parts, ie,

$$
\begin{aligned}
\operatorname{Thick}_{\varepsilon}(\mathbf{S}) & =\{x \in \dot{S} \mid \rho(x) \geq \varepsilon\} \\
\operatorname{Thin}_{\varepsilon}(\mathbf{S}) & =\underline{\{x \in \dot{S} \mid \rho(x)<\varepsilon\}},
\end{aligned}
$$

where $\rho(x)$ denotes the injectivity radius of the metric $h^{j, M}$ at the point $x \in \dot{S}$. It is a remarkable fact of the hyperbolic geometry that there exists a universal constant $\varepsilon_{0}=\sinh ^{-1} 1=\ln (1+\sqrt{2})$ such that for any $\varepsilon<\varepsilon_{0}$ each component $C$ of $\operatorname{Thin}_{\varepsilon}(\mathbf{S})$ is conformally equivalent either to a finite cylinder $[-L, L] \times S^{1}$ if the component $C$ is not adjacent to a puncture, or to the punctured disc $D^{2} \backslash 0 \cong[0, \infty) \times S^{1}$ otherwise, see for example [20]. Each compact component $C$ of the thin part contains a unique closed geodesic of length equal $2 \rho(C)$, which will be denoted by $\Gamma_{C}$. Here $\rho(C)=\inf _{x \in C} \rho(x)$. When considering $\varepsilon$-thick-thin decompositions we will always assume that $\varepsilon$ is chosen smaller than $\varepsilon_{0}$.

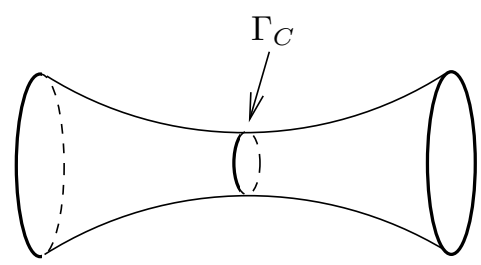

(a)

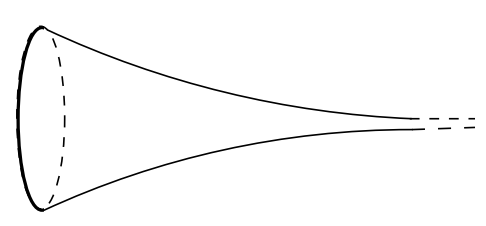

(b)

Figure 2: Thin parts non-adjacent $(a)$ and adjacent $(b)$ to a puncture

\subsection{Compactification of a punctured Riemann surface}

For each marked point $z \in M$ of a Riemann surface $\mathbf{S}=(S, j, M)$ we define the surface $S^{z}$ with boundary as an oriented blow-up of $S$ at the point $z$. Thus, $S^{z}$ is the circle compactification of $S \backslash\{z\}$ and it is a compact surface bounded by the circle $\Gamma_{z}=\left(T_{z} S \backslash 0\right) / \mathbb{R}_{+}^{*}$, where $\mathbb{R}_{+}^{*}=(0, \infty)$. The conformal structure $j$ defines an action of the circle $S^{1}=\mathbb{R} / \mathbb{Z}$ on $\Gamma_{z}$, and hence allows us to canonically metrize $\Gamma_{z}$. The canonical projection $\pi: S^{z} \rightarrow S$ sends the circle $\Gamma_{z}$ to the point $z$ and maps Int $S^{z}$ diffeomorphically to $S \backslash\{z\}$. Similarly, given a finite set $M=\left\{z_{1}, \ldots, z_{k}\right\}$ of punctures we define a blow-up surface $S^{M}$ having $k$ boundary components $\Gamma_{1}, \ldots, \Gamma_{k}$. It comes with the projection $\pi: S^{M} \rightarrow S$ 
which collapses the boundary circles $\Gamma_{1}, \ldots, \Gamma_{k}$ to the points $z_{1}, \ldots, z_{k}$ and maps Int $S^{M}$ diffeomorphically to $\dot{S}=S \backslash M$. A Riemann surface $\mathbf{S}$ is called $\varepsilon$-thick if $\operatorname{Thin}_{\varepsilon}(\mathbf{S})$ consists only of non-compact (ie, adjacent to punctures) components. It is not difficult to see that the subspace $\mathcal{M}_{g, \mu}^{\varepsilon} \subset \mathcal{M}_{g, \mu}$ of moduli of $\varepsilon$-thick Riemann surfaces of signature $(g, \mu)$ is compact with respect to its natural topology. However, to compactify the moduli space of all hyperbolic metrics on $S \backslash M$ one has to add degenerate metrics, or metrics with interior cusps, if the length of the closed geodesics $\Gamma_{C}$ in one or several components of the thin parts converge to 0 .

\subsection{Stable nodal Riemann surfaces}

We introduce a notion of a nodal Riemann surface. Suppose we are given a possibly disconnected Riemann surface $\mathbf{S}=(S, j, M, D)$ whose set of marked points is presented as a disjoint union of sets $M$ and $D$, where the cardinality of the set $D$ is even. The marked points from $D$, which are called special, are organized in pairs, $D=\left\{\bar{d}_{1}, \underline{d}_{1}, \bar{d}_{2}, \underline{d}_{2}, \ldots, \bar{d}_{k}, \underline{d}_{k}\right\}$. The nodal Riemann surface is the equivalence class of surfaces $(S, j, M, D)$ under the additional equivalence relations which make

- each pair $\left(\bar{d}_{i}, \underline{d}_{i}\right)$, for $i=1, \ldots, k$, and

- the set of all special pairs $\left\{\left(\bar{d}_{1}, \underline{d}_{1}\right),\left(\bar{d}_{2}, \underline{d}_{2}\right), \ldots,\left(\bar{d}_{k}, \underline{d}_{k}\right)\right\}$

unordered. For notational convenience we will still denote the nodal curve by $\mathbf{S}=(S, j, M, D)$, but one should remember that the numeration of pairs of of points in $D$, and the ordering of each pair is not part of the structure. The nodal curve is called stable if the stability condition (3) is satisfied for each component of the surface $S$ marked by the points from $M \cup D$. With a nodal surface $\mathbf{S}$ we can associate the following singular surface with double points,

$$
\widehat{S}_{D}=S /\left\{\bar{d}_{i} \sim \underline{d}_{i} ; i=1, \ldots, k\right\} .
$$

We shall call the identified points $\bar{d}_{i} \sim \underline{d}_{i}$ a node. The nodal surface $\mathbf{S}$ is called connected if the singular surface $\widehat{S}_{D}$ is connected. If the nodal surface $\mathbf{S}$ is connected then its arithmetic genus $g$ (compatible with the definition of the deformation $S^{D, r}$ below) is defined as

$$
g=\frac{1}{2} \# D-b_{0}+\sum_{1}^{b_{0}} g_{i}+1,
$$

where $\# D=2 k$ is the cardinality of $D$, and $b_{0}$ is the number of connected components of the surface $S$, and $\sum_{1}^{b_{0}} g_{i}$ is the sum of the genera of the connected components of $S$. The signature of a nodal curve $\mathbf{S}=(S, j, M, D)$ is 
the pair $(g, \mu)$ where $g$ is the arithmetic genus and $\mu=\# M$. A stable nodal Riemann surface $\mathbf{S}=(S, j, M, D)$ is called decorated if for each special pair there is chosen an orientation reversing orthogonal map

$$
r_{i}: \bar{\Gamma}_{i}=\left(T_{\bar{d}_{i}}(S) \backslash 0\right) / \mathbb{R}_{+}^{*} \rightarrow \underline{\Gamma}_{i}=\left(T_{\underline{d}_{i}}(S) \backslash 0\right) / \mathbb{R}_{+}^{*} .
$$

Orthogonal orientation reversing requires $r\left(e^{i \vartheta} z\right)=e^{-i \vartheta} r(z)$ for all $z \in \bar{\Gamma}_{i}$.

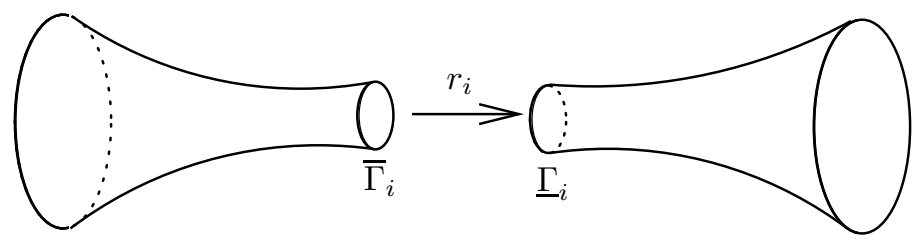

Figure 3: The decoration

We will also consider partially decorated surfaces if the maps $r_{i}$ are given only for a certain subset $D^{\prime}$ of special double points. An equivalence of decorated nodal surfaces must respect the decorating maps $r_{i}$. The moduli spaces of stable connected Riemann nodal surfaces, and decorated stable connected nodal surfaces of signature $(g, \mu)$ will be denoted by $\overline{\mathcal{M}}_{g, \mu}$ and $\overline{\mathcal{M}}_{g, \mu}^{\$}$, respectively. Note that the moduli space $\mathcal{M}_{g, \mu}$ of smooth Riemann surfaces, ie, surfaces with the empty set $D$ of double points, is contained in both spaces $\overline{\mathcal{M}}_{g, \mu}$ and $\overline{\mathcal{M}}_{g, \mu}^{\$}$, so that the natural projection $\overline{\mathcal{M}}_{g, \mu}^{\$} \rightarrow \overline{\mathcal{M}}_{g, \mu}$ is the identity map on $\mathcal{M}_{g, \mu} \subset \overline{\mathcal{M}}_{g, \mu}^{\$}$. Let us consider the oriented blow-up $S^{D}$ at the points of $D$ as described in Section 4.3 above. The circles $\bar{\Gamma}_{i}$ and $\underline{\Gamma}_{i}$ introduced in (7) serve as the boundary circles corresponding to the points $\bar{d}_{i}, \underline{d}_{i} \in D$. The canonical projection $\pi: S^{D} \rightarrow S$, which collapses the circles $\bar{\Gamma}_{i}$ and $\underline{\Gamma}_{i}$ to the points $\bar{d}_{i}$ and $\underline{d}_{i}$, induces on Int $S^{D}$ a conformal structure. The smooth structure of Int $S^{D}=S \backslash D$ extends to $S^{D}$, while the extended conformal structure degenerates along the boundary circles $\bar{\Gamma}_{i}$ and $\underline{\Gamma}_{i}$. Given a decorated nodal surface $(\mathbf{S}, r)$, where $r=\left(r_{1}, \ldots, r_{k}\right)$, we can glue $\overline{\bar{\Gamma}}_{i}$ and $\underline{\Gamma}_{i}$ by means of the mappings $r_{i}$, for $i=1, \ldots, k$, to obtain a closed surface $S^{D, r}$. As it was pointed out in Section 4.3, the special circles $\Gamma_{i}=\left\{\bar{\Gamma}_{i} \sim \underline{\Gamma}_{i}\right\}$ are endowed with the canonical metric. The genus of the surface $S^{D, r}$ is equal to the arithmetic genus of $\mathbf{S}$. There exists a canonical projection $p: S^{D, r} \rightarrow \widehat{S}_{D}$ which projects the circle $\Gamma_{i}=\left\{\bar{\Gamma}_{i}, \underline{\Gamma}_{i}\right\}$ to the double point $d_{i}=\left\{\bar{d}_{i}, \underline{d}_{i}\right\}$. The projection $p$ induces on the surface $S^{D, r}$ a conformal structure, still denoted by $j$, in the complement of the special circles $\Gamma_{i}$. The continuous extension of $j$ to $S^{D, r}$ degenerates along the special circles $\Gamma_{i}$. The uniformizing metric $h^{j, M \cup D}$ can also be lifted to a 
metric $h^{\mathrm{S}}$ on $\dot{S}^{D, r}=S^{D, r} \backslash M$. The lifted metric degenerates along each circle $\Gamma_{i}$, namely the length of $\Gamma_{i}$ is 0 , and the distance of $\Gamma_{i}$ to any other point in $\dot{S}^{D, r}$ is infinite. However, we can still speak about geodesics on $\dot{S}^{D, r}$ orthogonal to $\Gamma_{i}$. Namely, two geodesic rays, whose asymptotic directions at the cusps $\bar{d}_{i}$ and $\underline{d}_{i}$ are related via the map $r_{i}$, correspond to a compact geodesic interval in $S^{\bar{D}, r}$, which orthogonally intersects the circle $\Gamma_{i}$. Notice that the smooth structure on the oriented blow up surface $S^{D}$ with boundary is compatible with some smooth structure on $S^{D, r}$. Moreover, using the hyperbolic metric one can make this choice canonical. However, this smooth structure will be irrelevant for us and we will not discuss here the details of its construction. It will be convenient for us to view $\operatorname{Thin}_{\varepsilon}(\mathbf{S})$ and $\operatorname{Thick}_{\varepsilon}(\mathbf{S})$ as subsets of $\dot{S}^{D, r}$. This interpretation provides us with a compactification of non-compact components of $\operatorname{Thin}_{\varepsilon}(\mathbf{S})$ not adjacent to points from $M$. Every compact component $C$ of $\overline{\operatorname{Thin}_{\varepsilon}(\mathbf{S})} \subset S^{D, r}$ is a compact annulus. It contains either a closed geodesic $\Gamma_{C}$, or one of the special circles which will also be denoted by $\Gamma_{C}$. This special circle projects to a node as described above. The surface $S^{D, r}$ with all the endowed structures and the projection $S^{D, r} \rightarrow \widehat{S}_{D}$ is called the deformation of the decorated nodal surface $(\mathbf{S}, r)$. One can also define a partial deformation $S^{D^{\prime}, r^{\prime}}$ of $(\mathbf{S}, r)$ by splitting the set $D$ into a disjoint union $D=D^{\prime} \cup D^{\prime \prime}$ which respects the pairing structure, and then applying the above construction to $D^{\prime}$ while adjoining $D^{\prime \prime}$ to the set $M$ of the marked points.

\subsection{Topology of spaces $\overline{\mathcal{M}}_{g, \mu}$ and $\overline{\mathcal{M}}_{g, \mu}^{\$}$}

In this section we define the meaning of the convergence in the spaces $\overline{\mathcal{M}}_{g, \mu}$ and $\overline{\mathcal{M}}_{g, \mu}^{\$}$. The introduced topologies are compatible with certain metric structures on the spaces $\overline{\mathcal{M}}_{g, \mu}$ and $\overline{\mathcal{M}}_{g, \mu}^{\$}$ which we discuss in Appendix B.1. In particular, the introduced topologies are Hausdorff. Consider a sequence of decorated stable nodal marked Riemann surfaces

$$
\left(\mathbf{S}_{n}, r_{n}\right)=\left\{S_{n}, j_{n}, M_{n}, D_{n}, r_{n}\right\}, \quad n \geq 1 .
$$

The sequence $\left(\mathbf{S}_{n}, r_{n}\right)$ is said to converge to a decorated stable nodal surface

$$
(\mathbf{S}, r)=(S, j, M, D, r)
$$

if (for sufficiently large $n$ ) there exists a sequence of diffeomorphisms

$$
\varphi_{n}: S^{D, r} \rightarrow S_{n}^{D_{n}, r_{n}} \quad \text { with } \varphi_{n}(M)=M_{n}
$$

and such that the following conditions are satisfied. 
CRS1 For every $n \geq 1$, the images $\varphi_{n}\left(\Gamma_{i}\right)$ of the special circles $\Gamma_{i} \subset S^{D, r}$ for $i=1, \ldots, k$, are special circles or closed geodesics of the metrics $h^{j_{n}, M_{n} \cup D_{n}}$ on $\dot{S}^{D_{n}, r_{n}}$. Moreover, all special circles on $S^{D_{n}, r_{n}}$ are among these images.

CRS2 $h_{n} \rightarrow h^{\mathbf{S}}$ in $C_{\mathrm{loc}}^{\infty}\left(S^{D, r} \backslash\left(M \cup \bigcup_{1}^{k} \Gamma_{i}\right)\right)$, where $h_{n}=\varphi_{n}^{*} h^{j_{n}, M_{n} \cup D_{n}}$.

CRS3 Given a component $C$ of $\operatorname{Thin}_{\varepsilon}(\mathbf{S}) \subset \dot{S}^{D, r}$ which contains a special circle $\Gamma_{i}$ and given a point $c_{i} \in \Gamma_{i}$, we consider for every $n \geq 1$ the geodesic arc $\delta_{i}^{n}$ for the induced metric $h_{n}=\varphi_{n}^{*} h^{j_{n}, M_{n} \cup D_{n}}$ which intersects $\Gamma_{i}$ orthogonally at the point $c_{i}$, and whose ends are contained in the $\varepsilon^{-}$ thick part of the metric $h_{n}$. Then $\left(C \cap \delta_{i}^{n}\right)$ converges as $n \rightarrow \infty$ in $C^{0}$ to a continuous geodesic for the metric $h^{\mathbf{S}}$ which passes through the point $c_{i}$.

Remark 4.1 Let us point out that in view of the Uniformization Theorem, the condition CRS2 is equivalent to the condition

$$
\varphi_{n}^{*} j_{n} \rightarrow j \text { in } C_{\mathrm{loc}}^{\infty}\left(S^{D, r} \backslash\left(M \cup \bigcup_{1}^{k} \Gamma_{i}\right)\right) .
$$

Moreover, the Removable Singularity Theorem guarantees that the latter condition is equivalent to

$$
\varphi_{n}^{*} j_{n} \rightarrow j \text { in } C_{\mathrm{loc}}^{\infty}\left(S^{D, r} \backslash \bigcup_{1}^{k} \Gamma_{i}\right) .
$$

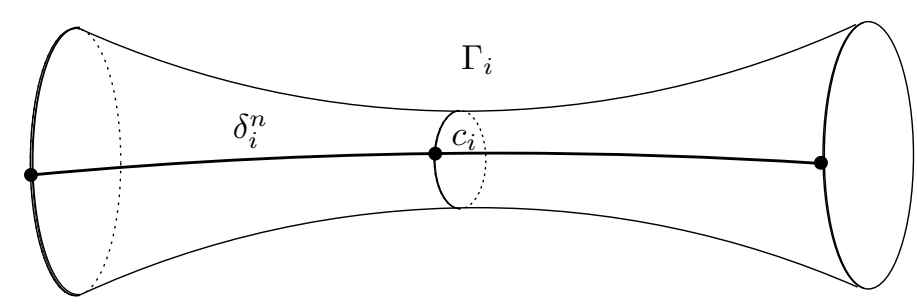

Figure 4: Illustration for property CR3

A sequence $\mathbf{S}_{n} \in \overline{\mathcal{M}}_{g, \mu}$ is said to converge to $\mathbf{S} \in \overline{\mathcal{M}}_{g, \mu}$ if there exists a sequence of decorations $r_{n}$ for $\mathbf{S}_{n}$ and a decoration $r$ of $\mathbf{S}$ such that $\left(\mathbf{S}_{n}, r_{n}\right)$ converges to $(\mathbf{S}, r)$ in $\overline{\mathcal{M}}_{g, \mu}^{\$}$. In other words the topology on $\overline{\mathcal{M}}_{g, \mu}$ is defined as the weakest topology on $\overline{\mathcal{M}}_{g, \mu}$ for which the projection $\overline{\mathcal{M}}_{g, \mu}^{\$} \rightarrow \overline{\mathcal{M}}_{g, \mu}$ is continuous. Note 
that the convergence in the space $\overline{\mathcal{M}}_{g, \mu}$ can also be defined by the properties CRS1 and CRS2 above.

Theorem 4.2 (Deligne-Mumford [2], Wolpert [28]) The spaces $\overline{\mathcal{M}}_{g, \mu}$ and $\overline{\mathcal{M}}_{g, \mu}^{\$}$ are compact metric spaces, and serve as the compactifications of the space $\mathcal{M}_{g, \mu}$, ie, they coincide with the closure of $\mathcal{M}_{g, \mu}$ viewed as a subspace of $\overline{\mathcal{M}}_{g, \mu}$ and of $\overline{\mathcal{M}}_{g, \mu}^{\$}$, respectively. In particular, every sequence of smooth marked Riemann surfaces $\mathbf{S}_{n}=\left(S_{n}, j_{n}, M_{n}\right)$ of signature $(g, \mu)$ has a subsequence which converges to a decorated nodal curve $\mathbf{S}=(S, j, M, D, r)$ of signature $(g, \mu)$.

The next proposition illustrates the geometry of the Deligne-Mumford convergence in a special case when one varies the configuration of the marked points. It follows from the definition of this convergence and from scaling operations on the limit surface.

Proposition 4.3 Let $\mathbf{S}_{n}=\left(S_{n}, j_{n}, M_{n}, D_{n}\right)$ be a sequence of smooth marked nodal Riemann surfaces of signature $(g, \mu)$ which converges to a nodal curve $\mathbf{S}=(S, j, M, D)$ of signature $(g, \mu)$. Suppose that for each $n \geq 1$, we are given a pair of points $Y_{n}=\left\{y_{n}^{(1)}, y_{n}^{(2)}\right\} \subset S_{n} \backslash\left(M_{n} \cup D_{n}\right)$ such that

$$
\operatorname{dist}_{n}\left(y_{n}^{(1)}, y_{n}^{(2)}\right) \underset{n \rightarrow \infty}{\longrightarrow} 0 \text {. }
$$

Here dist ${ }_{n}$ is the distance with respect to the hyperbolic metric $h^{j_{n}, M_{n} \cup D_{n}}$ on $S_{n} \backslash\left(M_{n} \cup D_{n}\right)$. Suppose, in addition, that there is a sequence $R_{n} \underset{n \rightarrow \infty}{\rightarrow} \infty$ such that there exist injective holomorphic maps $\varphi_{n}: D_{R_{n}} \rightarrow S_{n} \backslash\left(M_{n} \cup D_{n}\right)$, where $D_{R_{n}}$ denotes the disc $\left\{|z| \leq R_{n}\right\} \subset \mathbb{C}$, satisfying $\varphi_{n}(0)=y_{n}^{(1)}$ and $\varphi_{n}(1)=y_{n}^{(2)}$. Then there exists a subsequence of the new sequence $\mathbf{S}_{n}^{\prime}=$ $\left(S_{n}, j_{n}, M_{n} \cup Y_{n}, D_{n}\right)$ which converges to a nodal curve $\mathbf{S}^{\prime}=\left(S^{\prime}, j^{\prime}, M^{\prime}, D^{\prime}\right)$ of signature $(g, \mu+2)$, which has one or two additional spherical components. One of these components contains the marked points $y^{(1)}$ and $y^{(2)}$ which correspond to the sequences $y_{n}^{(1)}$ and $y_{n}^{(2)}$. The possible cases are illustrated by Figure 5 and described in detail below.

Let $r_{n}, r$ be some decorations of $\mathbf{S}_{n}$ and $\mathbf{S}$ such that $\left(\mathbf{S}_{n}, r_{n}\right) \underset{n \rightarrow \infty}{\rightarrow}(\mathbf{S}, r)$. Let $\varphi_{n}: S^{D, r} \rightarrow S_{n}^{D_{n}, r_{n}}$ be the sequence of diffeomorphisms guaranteed by the definition of the convergence of decorated Riemann surfaces $\mathbf{S}_{n} \rightarrow \mathbf{S}$. Let $\widehat{S}_{D}$ be the singular surface with double points as defined in (5), and $\pi: S^{D, r} \rightarrow \widehat{S}_{D}$ the canonical projection. Set $Z_{n}=\pi\left(\varphi^{-1}\left(Y_{n}\right)\right) \subset \widehat{S}_{D}$. Then the following scenarios are possible: 
i)

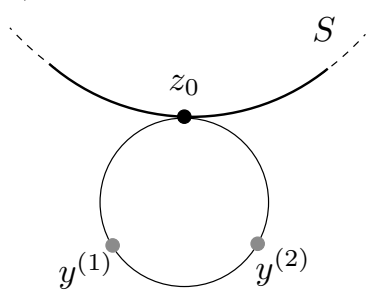

ii)

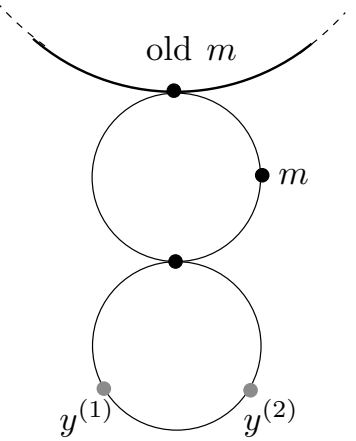

iii)

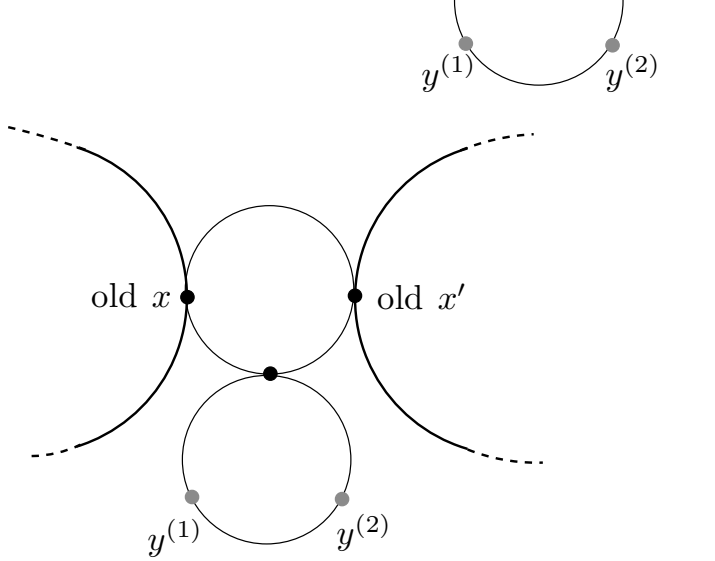

Figure 5: The possible configuration of spherical bubbles appearing in Proposition 4.3.

i) The points $z_{n}^{(1)}, z_{n}^{(2)} \in Z_{n}$ converge to a point $z_{0}$ which does not belong to $M$ nor to $D$. Then the limit $\mathbf{S}^{\prime}$ of $\mathbf{S}_{n}^{\prime}$ has an extra sphere attached at $z_{0}$ on which there are two marked points $y^{(1)}, y^{(2)}$, see Figure 5i).

ii) The points $z_{n}^{(1)}, z_{n}^{(2)} \in Z_{n}$ converge to a marked point $m \in M$. In this case the limit $\mathbf{S}^{\prime}$ is $\mathbf{S}$ with two extra spheres $T_{1}$ and $T_{2}$ attached. The sphere $T_{1}$ is attached at its $\infty$ to the "old" $m$ and has $m$ as its $0 . T_{2}$ is attached at its $\infty$ to the point $1 \in T_{1}$ and contains the marked points $y^{(1)}$ and $y^{(2)}$, see Figure 5ii).

iii) The points in $Z_{n}$ converge to a double point $d$ which corresponds to the node $\left\{x, x^{\prime}\right\} \in D$. Then between the nodal points $x$ and $x^{\prime}$ a new sphere $T_{1}$ is inserted, say attached at its $\infty$ to $x$ and at 0 to $x^{\prime}$. At the point 1 a second sphere $T_{2}$ carrying two marked points $y^{(1)}, y^{(2)}$ is attached, see Figure 5iii). 


\section{Holomorphic curves in cylindrical almost complex manifolds}

\subsection{Gromov-Schwarz and monotonicity}

We begin by recalling two important analytic results about $J$-holomorphic maps (see [8] and $[25,20]$ for the proofs).

Lemma 5.1 (Gromov-Schwarz) Let $f: D^{2}(1) \rightarrow W$ be a holomorphic disk in an almost complex manifold $W$ whose structure $J$ is tamed by an exact symplectic form. If the image of $f$ is contained in a compact set $K \subset W$, then

$$
\left\|\nabla^{k} f(x)\right\| \leq C(K, k) \quad \text { for all } x \in D^{2}(1 / 2),
$$

for every $k \geq 1$ where the constants do not depend on $f$.

Note that locally every $J$ is tamed by some symplectic form, and hence Lemma 5.1 holds for sufficiently small compact sets in every almost complex manifold.

Lemma 5.2 (Monotonicity) Let $(W, J)$ be a compact almost complex manifold and suppose $J$ to be tamed by $\omega$. Then there exists a positive constant $C_{0}$ having the following property. Assume that $f:(S, j) \rightarrow(W, J)$ is a compact $J$-holomorphic curve with boundary and choose $s_{0} \in S \backslash \partial S$ and $r$ smaller that the injectivity radius of $W$. If the boundary $f(\partial S)$ is contained in the complement of the $r$-ball $B_{r}\left(f\left(s_{0}\right)\right) \subset W$, then the area of $f$ inside of the ball $\bar{B}_{r}\left(f\left(s_{0}\right)\right)$ satisfies

$$
\int_{f(S) \cap B_{r}\left(f\left(s_{0}\right)\right)} \omega \geq C_{0} r^{2} .
$$

\section{$5.2 \bar{\partial}$-equation on cylindrical manifolds}

Let $(W=\mathbb{R} \times V, J)$ be a cylindrical almost complex manifold and let $\left(\xi, J_{\xi}\right)$, $\mathbf{R} \in T V$ and $\lambda$ be the corresponding CR-structure, the vector field transversal to $\xi$ and the 1 -form on $V$ determined by the conditions $\xi=\{\lambda=0\}$ and $\lambda(R)=1$. We will denote by $p_{\mathbb{R}}$ and $p_{V}$ the projections to the first and the second factor of $W$, and by $\pi$ the projection $T V \rightarrow \xi$ along the direction of the field $\mathbf{R}$. Let us agree on the following notational convention. We shall use capital roman letters to denote maps to $W$, and corresponding small letters for their projections to $V$, eg, if $F$ is a map to $W$, then $p_{V} \circ F$ will be denoted by $f$. The $\mathbb{R}$-component $p_{\mathbb{R}} \circ F$ of $F$ will usually be denoted by $a$, or $a_{F}$ if necessary. 
Given a Riemann surface $(\Sigma, j)$, the $\bar{\partial}$-equation defines the holomorphic maps (or curves) $F=(a, f):(\Sigma, j) \rightarrow(W=\mathbb{R} \times V, J)$ as solutions of the equation

$$
T F \circ j=J \circ T F \text {. }
$$

For our distinguished structure $J$, the equation takes the form

$$
\begin{gathered}
\pi \circ d f \circ j=J \circ \pi \circ d f \\
d a=\left(f^{*} \lambda\right) \circ j .
\end{gathered}
$$

Notice, that the second equation just means that the form $f^{*} \lambda \circ j$ is exact on $\Sigma$ and that the function $a$ is a primitive of the 1 -form $f^{*} \lambda \circ j$. Thus the holomorphicity condition for $F=(a, f)$ is essentially just a condition on its $V$ component $f$. If $f$ satisfies the first of the equations (8) and the form $\left(u^{*} \lambda\right) \circ j$ is exact then the coordinate $a$ can be reconstructed uniquely up to an additive constant on each connected component of $\Sigma$. We will call the map $f: \Sigma \rightarrow V$ a holomorphic curve in $V$ if $f$ satisfies the first of the equations (8) and the form $\left(f^{*} \lambda\right) \circ j$ is exact.

\section{$5.3 \quad$ Energy}

A crucial assumption in Gromov's compactness theorem for holomorphic curves in a compact symplectic manifold is the finiteness of the area. However, the area of a non-compact proper holomorphic curve in a cylindrical manifold is never finite with respect to any complete metric. Moreover, in the contact case, or more generally in the case when the taming form $\omega$ is exact, there are no non-constant compact holomorphic curves. We define below another quantity, called energy, which serves as a substitute for the area in this case. Suppose that the cylindrical almost complex structure $J$ is adjusted to a closed form $\omega$. Let us define the $\omega$-energy $E(F)$ of the holomorphic map $F=(a, f):(S, j) \rightarrow$ $(\mathbb{R} \times V, J)$ by the formula

$$
E_{\omega}(F)=\int_{S} f^{*} \omega
$$

and the $\lambda$-energy by the formula

$$
E_{\lambda}(F)=\sup _{\phi \in \mathcal{C}} \int_{S}(\phi \circ a) d a \wedge f^{*} \lambda,
$$

where the supremum is taken over the set $\mathcal{C}$ of all non-negative $C^{\infty}$-functions $\phi: \mathbb{R} \rightarrow \mathbb{R}$ having compact support and satisfying the condition

$$
\int_{\mathbb{R}} \phi(s) d s=1 .
$$

Geometry 8 Topology, Volume 7 (2003) 
It is important to observe that the $\omega$-energy is additive, while the $\lambda$-energy is not. Finally, the energy of $F$ is defined as the sum

$$
E(F)=E_{\omega}(F)+E_{\lambda}(F) .
$$

Note that the $\omega$-energy $E_{\omega}(F)$ depends only on the $V$-component $f$ ofthe map $F$. The following inequality is a straightforward consequence of the definition of the $\lambda$-energy $E_{\lambda}(F)$.

Lemma 5.3 For any holomorphic curve $F=(a, f):(S, j) \rightarrow(\mathbb{R} \times V, J)$ and any non-critical level $\Gamma_{c}=\{a=c\}$,

$$
\left|\int_{\Gamma_{c}} \lambda\right| \leq E_{\lambda}(F)
$$

The adjustment condition implies the following lemma.

Lemma 5.4 For any holomorphic curve $F$ we have $E_{\omega}(F), E_{\lambda}(F) \geq 0$. Moreover, we have $\int_{S}(\phi \circ a) d a \wedge f^{*} \lambda \geq 0$ for any $\phi \in \mathcal{C}$. If $E_{\omega}(F)=0$, then the image $f(S)$ is contained in a trajectory of the vector field $\mathbf{R}$.

\subsection{Properties of holomorphic cylinders}

We begin with a version of the Removable Singularity Theorem in our context.

Lemma 5.5 Let $F=(a, f):\left(D^{2} \backslash\{0\}, j\right) \rightarrow(\mathbb{R} \times V, J)$ be a holomorphic map with $E(F)<\infty$. Suppose that the image of $F$ is contained in a compact subset $K$ of $\mathbb{R} \times V$. Then $F$ extends continuously to a holomorphic map $\widetilde{F}:\left(D^{2}, j\right) \rightarrow(\mathbb{R} \times V, J)$ with $E(\widetilde{F})=E(F)$.

Proof Choose $\phi \in \mathcal{C}$ such that $\phi(t)>0$ for all $(t, x) \in K$. Then, the (not necessarily closed) 2 -form $\Omega=\pi^{*} \omega+\phi(t) d t \wedge \lambda$ is non-degenerate on $K$ and tames $J$. Thus the energy bound for $F$ implies its area bound, and hence, we can apply the usual Removable Singularity Theorem (see for example [27]).

The next proposition, proven in $[12,17]$ in the non-degenerate case and in [1] in the Morse-Bott case, describes the behavior of finite energy holomorphic curves near the punctures in the case of non-removable singularities. 
Proposition 5.6 Suppose that the vector field $\mathbf{R}$ is of Morse, or Morse-Bott type. Let $F=(a, f): \mathbb{R}^{+} \times \mathbb{R} / \mathbb{Z} \rightarrow(\mathbb{R} \times V, J)$ be a holomorphic map of finite energy $E(F)<\infty$. Suppose that the image of $F$ is unbounded in $\mathbb{R} \times V$. Then there exist a number $T \neq 0$ and a periodic orbit $\gamma$ of $\mathbf{R}$ of period $|T|$ such that

$$
\lim _{s \rightarrow \infty} f(s, t)=\gamma(T t) \quad \text { and } \quad \lim _{s \rightarrow \infty} \frac{a(s, t)}{s}=T \quad \text { in } \quad C^{\infty}\left(S^{1}\right) .
$$

Proposition 5.7 Given $E_{0}, \varepsilon>0$, there exist constants $\sigma, c>0$ such that for every $R>c$ and every holomorphic cylinder $F=(a, f):[-R, R] \times S^{1} \rightarrow \mathbb{R} \times U$ satisfying the inequalities

$$
E_{\omega}(F) \leq \sigma \quad \text { and } \quad E(F) \leq E_{0},
$$

we have

$$
f(s, t) \in B_{\varepsilon}(f(0, t))
$$

for all $s \in[-R+c, R-c]$ and all $t \in \mathbb{R}$.

More precisely, there exists either a periodic orbit $\gamma$ of the vector field $\mathbf{R}$ on $V$ having period $T>0$ such that $f(s, t) \in B_{\varepsilon}(\gamma(T t))$ or a point $p^{*} \in \mathbb{R} \times V$ such that $F(s, t) \in B_{\varepsilon}\left(p^{*}\right)$ for all $s \in[-R+c, R-c]$ and all $t \in \mathbb{R}$. Hence a long holomorphic cylinder having small $\omega$-energy is either close to a trivial cylinder over a periodic orbit of the vector field $\mathbf{R}$, or close to a constant map. The proof in the non-degenerate case can be found in in [18]. In the Morse-Bott case the proof is given in Appendix A.

\subsection{Proper holomorphic maps of punctured Riemann surfaces}

Proposition 5.6 implies:

Proposition 5.8 Let $(S, j)$ be a closed Riemann surface and let

$$
Z=\left\{z_{1}, \ldots, z_{k}\right\} \subset S
$$

be a set of punctures. Every holomorphic map $F=(a, f):(S \backslash Z, j) \rightarrow \mathbb{R} \times V$ of finite energy and without removable singularities is asymptotically cylindrical near each puncture $z_{i}$ over a periodic orbit $\gamma_{i} \in \mathcal{P}$.

The puncture $z_{i}$ is called positive or negative depending on the sign of the coordinate function $a$ when approaching the puncture. Notice that the change of the holomorphic coordinates near the punctures affects only the choice of the origin on the orbit $\gamma_{i}$; the parametrization of the asymptotic orbits induced 
by the holomorphic polar coordinates remains otherwise the same. Hence, the orientation induced on $\gamma_{i}$ by the holomorphic coordinates coincides with the orientation defined by the vector field $\mathbf{R}$ if and only if the puncture is positive.

Remark 5.9 In the situation when $V$ is as in Example 2.2, a holomorphic map $F=(a, f):(S \backslash Z, j) \rightarrow \mathbb{R} \times V$ can be extended via the Removable Singularity Theorem into a holomorphic map $\bar{F}: S \rightarrow \bar{W}$, where $\bar{W}$ is the projectivization of the complex line bundle associated with the circle bundle $V \rightarrow M$. Punctures $z_{j}$ are then mapped by $\bar{F}$ to the divisors of $\bar{W}$ which correspond to 0 - and $\infty$-sections $M_{0}, M_{\infty} \subset \bar{W}$. The multiplicity $k_{j}$ of the asymptotic orbit of $\mathbf{R}$ corresponding to a puncture $z_{j} \in S$ equals $t_{j}+1$ where $t_{j}$ is the order of tangency of $\bar{F}$ to $M_{0} \cup M_{\infty}$ at $z_{j}$. This observation explains why the compactness theorems proven in $[21,24,23]$ follow from the results of this paper.

In what follows we will only consider holomorphic maps of Riemann surfaces which are conformally equivalent to a compact Riemann surface $(S, j)$ with punctures $Z=\left\{z_{1}, \ldots, z_{k}\right\}$. Let $S^{Z}$ be the oriented blow-up of $S$ at the points of $Z$, as it is defined in Section 4.3 above. Thus $S^{Z}$ is a compact surface with boundary consisting of circles $\Gamma_{1}, \ldots, \Gamma_{k}$. Each of these circles is endowed with a canonical $S^{1}$-action and we denote by $\varphi_{j}: S^{1} \rightarrow \Gamma_{j}$ the canonical (up to a choice of the base point) parametrization of the boundary circle $\Gamma_{j}$, for $j=1, \ldots, k$. Proposition 5.8 can be equivalently reformulated as follows.

Proposition 5.10 Let $F=(a, f):(S \backslash Z, j) \rightarrow(\mathbb{R} \times V, J)$ be a finite energy holomorphic map without removable singularities. Then the map $f: S \backslash Z \rightarrow V$ extends to a continuous map $\bar{f}: S^{Z} \rightarrow V$ satisfying

$$
\bar{f}\left(\varphi_{j}\left(e^{i t}\right)\right)=\gamma_{j}( \pm T t),
$$

where $\gamma_{j}: S^{1}=\mathbb{R} / \mathbb{Z} \rightarrow V$ is a periodic orbit of the vector field $\mathbf{R}$ of period $T$, parametrized by the vector field $\mathbf{R}$. The sign in the formula (11) coincides with the sign of the puncture $z_{j}$.

We will call the map $\bar{f}: S^{Z} \rightarrow V$ the compactification of the map $f$.

\subsection{Bubbling lemma}

Lemma 5.11 There exists a constant $\hbar$ depending only on $(V, J)$ so that the following holds true. Consider a sequence $F_{n}:=\left(a_{n}, f_{n}\right): D \rightarrow(\mathbb{R} \times V, J)$ 
of $J$-holomorphic maps of the unit disc $D=\{|z|<1\} \subset \mathbb{C}$ to $V$ satisfying $E\left(F_{n}\right) \leq C$ for some constant $C$ and such that $a_{n}(0)=0$. Fix a Riemannian metric on $V$. Suppose that $\left\|\nabla F_{n}(0)\right\| \rightarrow \infty$ as $n \rightarrow \infty$. Then there exists a sequence of points $y_{n} \in D$ converging to 0 , and sequences of positive numbers $c_{n}, R_{n} \rightarrow \infty$ as $n \rightarrow \infty$ such that $\left\|y_{n}\right\|+c_{n}^{-1} R_{n}<1$ and the rescaled maps

$$
\begin{aligned}
F_{n}^{0}: D_{R_{n}}=\left\{|z|<R_{n}\right\} & \rightarrow(\mathbb{R} \times V, J), \\
z & \mapsto F_{n}\left(y_{n}+c_{n}^{-1} z\right),
\end{aligned}
$$

converge in $C_{\text {loc }}^{\infty}(\mathbb{C})$ to a holomorphic map $F^{0}: \mathbb{C} \rightarrow \mathbb{R} \times V$ which satisfies the conditions

$$
E\left(F^{0}\right) \leq C \text { and } E_{\omega}\left(F^{0}\right)>\hbar .
$$

Moreover, this map is either a holomorphic sphere or a holomorphic plane $\mathbb{C}$ asymptotic as $|z| \rightarrow \infty$ to a periodic orbit of the vector field $\mathbf{R}$. (To be precise we mean in the first case that $F^{0}$ smoothly extends to $\left.S^{2}=\mathbb{C} \cup\{\infty\}\right)$.

For the proof of Lemma 5.11 we shall need the following lemma from [10].

Lemma 5.12 Let $(X, d)$ be a complete metric space, $f: X \rightarrow \mathbb{R}$ be a nonnegative continuous function, $x \in X$, and $\delta>0$. Then there exist $y \in X$ and a positive number $\varepsilon \leq \delta$ such that

$$
d(x, y)<2 \delta, \quad \sup _{B_{\varepsilon}(y)} f \leq 2 f(y), \quad \varepsilon f(y) \geq \delta f(x) .
$$

Proof of Lemma 5.11 Choose $\delta_{n}>0$ such that

$$
\delta_{n} \rightarrow 0, \quad \text { and } \quad \delta_{n}\left\|\nabla F_{n}(0)\right\| \rightarrow \infty .
$$

Applying Lemma 5.12, we obtain new sequences $y_{n} \in S$ and $0<\varepsilon_{n} \leq \delta_{n}$ such that $y_{n} \rightarrow x$ and

$$
\sup _{\left|z-y_{n}\right| \leq \varepsilon}\left\|\nabla F_{n}(z)\right\| \leq 2\left\|\nabla F_{n}\left(y_{n}\right)\right\|, \quad \varepsilon_{n}\left\|\nabla F_{n}\left(y_{n}\right)\right\| \rightarrow \infty .
$$

Introduce $c_{n}=\left\|\nabla F_{n}\left(y_{n}\right)\right\|$ and $R_{n}=\varepsilon_{n} c_{n}$. Notice that for sufficiently large $n$ we have $\left\|y_{n}\right\|+c_{n}^{-1} R_{n}<1$. We consider the rescaled maps

$$
F_{n}^{0}(z)=F_{n}\left(y_{n}+c_{n}^{-1} z\right) .
$$

This sequence has the following properties:

- $\sup _{D_{R_{n}}}\left\|\nabla F_{n}^{0}(z)\right\| \leq 2, \quad R_{n} \rightarrow \infty ;$

- $E\left(F_{n}^{0}\right) \leq E\left(F_{n}\right) \leq C$; 
- $\left\|\nabla F_{n}^{0}(0)\right\|=1$.

Now, by Ascoli-Arzela's theorem, we can extract a converging subsequence and thus we obtain a non-constant finite energy plane $F^{0}$. If the image of $F^{0}$ is contained in a compact subset of $\mathbb{R} \times V$, then by Lemma $5.5, F^{0}$ is a holomorphic sphere. Otherwise, we can apply Proposition 5.6 to deduce that $F^{0}$ is converging to an $\mathbf{R}$-orbit $\gamma$ for large radius. In both cases there is a constant $\hbar$ such that $E_{\omega}\left(F^{0}\right)>\hbar>0$. Indeed, otherwise we could get a sequence $F^{i}$ of holomorphic planes satisfying $E_{\omega}\left(F^{i}\right) \rightarrow 0$ as $i \rightarrow \infty$, having a uniform gradient bound and normalized by the condition $\left\|\nabla F^{i}(0)\right\|=1$. Then AscoliArzela's theorem would imply the existence of a non-constant limit holomorphic plane $F^{\infty}: \mathbb{C} \rightarrow \mathbb{R} \times V$ satisfying $E_{\omega}\left(F^{\infty}\right)=0$ and $E\left(F^{\infty}\right)<+\infty$. In view of Lemma 5.4 we then conclude that $F^{\infty}(\mathbb{C})$ is contained in a cylinder over an orbit of $\mathbf{R}$, and hence must coincide with the universal covering of this cylinder. But this is impossible in view of finiteness of the energy $E\left(F^{\infty}\right)$. This finishes off the proof.

\subsection{The symmetric case}

The next lemma shows that for a symmetric $J$, and in particular in the contact case, the energies of holomorphic curves $F=(a, f)$ asymptotic to prescribed periodic orbits from $\mathcal{P}$ can be uniformly bounded in terms of the relative homology class represented by $f$.

Proposition 5.13 Let $(\mathbb{R} \times V, J)$ be a symmetric cylindrical almost complex structure adjusted to a closed 2-form $\omega$ on $V$. Suppose that the holomorphic curve $F:(S \backslash Z, j) \rightarrow(\mathbb{R} \times V, J)$ of finite energy is asymptotic at the positive punctures to the periodic orbits $\bar{\gamma}_{1}, \ldots, \bar{\gamma}_{k} \in \mathcal{P}$ and to the periodic orbits $\underline{\gamma}_{1}, \ldots, \underline{\gamma}_{l} \in \mathcal{P}$ at the negative punctures. Then there exists a positive constant $\bar{C}$ (which depends on $J, \lambda, \omega$ but not $F$ ) such that

$$
E(F) \leq C \int_{S} f^{*} \omega+\sum_{1}^{k} S\left(\bar{\gamma}_{i}\right) .
$$

In particular, the energies $E(F)$ are uniformly bounded for all $F$ for which $f$ represents a given homology class in $H_{2}\left(V, \bigcup \bar{\gamma}_{i} \cup \underline{\gamma}_{j}\right)$.

Proof First observe that

$$
\left|f^{*} d \lambda\right| \leq C f^{*} \omega
$$


Indeed, in the symmetric case $\mathbf{R}\lrcorner d \lambda=\mathbf{R}\lrcorner \omega=0$, and hence the value of both forms on any bivector $\sigma$ is equal to the value of these forms on the projection of $\sigma$ to $\xi$. But a complex direction of $J$ projects to a complex direction of $J_{\xi}$, and hence the inequality follows from the Wirtinger inequality due to the fact that $J_{\xi}$ is tamed by $\left.\omega\right|_{\xi}$. Given any function $\phi \in \mathcal{C}$ we find, using Stokes' formula,

$$
\begin{aligned}
\left|\int_{S}(\phi \circ a) d a \wedge f^{*} \lambda\right| & \leq\left|\int_{S} F^{*} d(\psi \lambda)\right|+\left|\int_{S}(\psi \circ a) f^{*} d \lambda\right| \\
& \leq \sum_{1}^{k} S\left(\bar{\gamma}_{i}\right)+\int_{S}\left|f^{*} d \lambda\right|,
\end{aligned}
$$

where $\psi(s)=\int_{-\infty}^{s} \phi(\sigma) d \sigma$. Using (13) and (14) we obtain

$$
E_{\lambda}(F)=\sup _{\phi \in \mathcal{C}} \int_{S}(\phi \circ a) d a \wedge f^{*} \lambda \leq \sum_{1}^{k} S\left(\bar{\gamma}_{i}\right)+C \int_{S} f^{*} \omega
$$

We will also need the following property of holomorphic cylinders with small boundary circles.

Lemma 5.14 Let $F_{n}=\left(a_{n}, f_{n}\right):[-n, n] \times S^{1} \rightarrow \mathbb{R} \times V$ be a family of holomorphic cylinders. Suppose that

$$
E_{\omega}\left(F_{n}\right) \rightarrow 0 \text { and }\left.\lim _{n \rightarrow \infty} F_{n}^{ \pm}\right|_{ \pm n \times S^{1}}=z_{ \pm} \in \mathbb{R} \times V \text { in } C^{1}\left(S^{1}\right),
$$

where $z_{ \pm}$are two points and where the maps $F_{n}^{ \pm}$differ from $F_{n}$ by a translation with a sequence of constants,

$$
F_{n}^{ \pm}=\left(a_{n}-c_{n}^{ \pm}, f_{n}\right)
$$

Then

$$
\left.\operatorname{diam} F_{n}\left([-n, n] \times S^{1}\right)\right) \rightarrow 0 \text { as } n \rightarrow \infty
$$

Proof The cylindrical almost complex structure $J$ on $\mathbb{R} \times V$ is tamed by an almost symplectic, ie, non-degenerate but not necessarily closed, differential 2form $\Omega=\omega+d g(t) \wedge \lambda$, where $g: \mathbb{R} \rightarrow(0, \varepsilon)$ is $C^{\infty}$-function with a positive derivative. The Monotonicity Lemma 5.2 implies the existence of a constant $C>0$ such that for a sufficiently small $\varepsilon>0$, for every holomorphic curve $S$ 
and every point $x \in S$ such that the ball $B_{\varepsilon}(x)$ does not intersect the boundary of $S$, we have (possibly after translating $S$ along the $\mathbb{R}$-direction) the inequality

$$
\int_{S \cap B_{\varepsilon}(x)} \Omega \geq C \varepsilon^{2} .
$$

Suppose that $\operatorname{diam} F_{n}\left([-n, n] \times S^{1}\right) \geq \delta>0$. Then there exists a point $y_{n} \in$ $[-n, n] \times S^{1}$ such that

$$
\operatorname{dist}\left(F_{n}\left(y_{n}\right), F_{n}\left(\partial\left([-n, n] \times S^{1}\right)\right)\right) \leq \frac{\delta}{2} .
$$

Choosing $\varepsilon<\delta / 2$ we conclude, after possibly translating the cylinder $F_{n}$, that

$$
\int_{[-n, n] \times S^{1}} F_{n}^{*} \Omega \geq \int_{[-n, n] \times S^{1} \cap F_{n}^{-1}\left(B_{\varepsilon}\left(F_{n}\left(y_{n}\right)\right)\right.} F_{n}^{*} \Omega \geq C \varepsilon^{2} .
$$

On the other hand, by Stokes' theorem,

$$
\int_{[-n, n] \times S^{1}} F_{n}^{*} \Omega=\int_{[-n, n] \times S^{1}} F_{n}^{*}(\omega-g(t) d \lambda)+\int_{F_{n}\left(-n \times S^{1} \cup n \times S^{1}\right)} g(t) \lambda .
$$

The second term on the right-hand side of this equality converges to 0 . On the other hand, the symmetry condition and the Wirtinger inequality imply that

$$
\int_{[-n, n] \times S^{1}}\left|F_{n}^{*} d \lambda\right| \leq C \int_{[-n, n] \times S^{1}} F_{n}^{*} \omega \leq C E_{\omega}\left(F_{n}\right) \rightarrow 0
$$

as $n \rightarrow \infty$. Hence, the right-hand side of (17) converges to 0 , which contradicts the positive lower bound (16) and completes the proof of the lemma.

\subsection{The contact case: relation between energy and contact area}

We consider in this section the contact case, ie, we assume that the 1 -form $\lambda$ implied by the definition of a cylindrical structure $J$ is a contact form, and $J$ is adjusted to $d \lambda=\omega$. We shall denote the $d \lambda$-energy $E_{d \lambda}(F)$ by $A(F)$ and call it the contact area.

Lemma 5.15 Let $F=(a, f):(S \backslash Z, j) \rightarrow(\mathbb{R} \times V, J)$ be a holomorphic map. Then the following two statements are equivalent,

(i) $A(F)<\infty$ and $F$ is a proper map;

(ii) $E(F)<\infty$ and $S$ has no removable punctures. 
Proof (i) $\Rightarrow$ (ii). By properness of $F$, the limit of the coordinate function $a$ near each puncture is either $+\infty$ or $-\infty$, and hence all the punctures can be divided into positive and negative punctures, according to the particular end of $\mathbb{R} \times V$ which the holomorphic curves approaches near the puncture. In a neighborhood $U$ of a puncture $p$, let $z$ be a complex coordinate vanishing at $p$. Let $D_{r}(p)=\{q \in U|| z(q) \mid \leq r\}$ and $C_{r}(p)=\partial D_{r}(p)$ oriented counterclockwise for a positive puncture $p$, and clockwise for a negative one. Consider $\int f^{*} \lambda$ as a function of $r$. It is increasing and bounded above (resp. decreasing $C_{r}(p)$

and bounded below) if the puncture is positive (resp. negative), since $d \lambda \geq 0$ on complex lines and $\int_{D_{r}(p)} d \lambda<C$. Hence $C_{r}(p)$ has a finite limit for $r \rightarrow 0$ for all, positive and negative punctures. Now, let $\phi \in \mathcal{C}$ and let $\phi_{n} \in \mathcal{C}$ such that $\phi_{n} \circ a=0$ in $D_{\frac{1}{n}}(p)$ for all punctures $p$. Such functions exist, by properness of $F$. Moreover, we can choose $\phi_{n}$ so that $\left\|\phi-\phi_{n}\right\|_{C^{0}}<\varepsilon_{n}$, with $\varepsilon_{n} \rightarrow 0$ for $n \rightarrow \infty$. We have

$$
\int_{S}\left(\phi_{n} \circ a\right) d a \wedge \lambda=\int_{S} F^{*} d\left(\psi_{n} \lambda\right)-\int_{S}\left(\psi_{n} \circ a\right) f^{*} d \lambda,
$$

where $\psi_{n}(s)=\int_{-\infty}^{s} \phi_{n}(\sigma) d \sigma$. Notice that $\psi_{n} \circ a=1$ in $D_{\frac{1}{n}}(p)$ when $p$ is a positive puncture and $\psi_{n} \circ a=0$ in $D_{\frac{1}{n}}(p)$ when $p$ is a negative one. By Stokes theorem,

$$
\int_{S} F^{*} d\left(\psi_{n} \lambda\right)=\lim _{r \rightarrow 0} \sum_{p} \int_{C_{r}(p)} f^{*} \lambda,
$$

where the sum is taken over all positive punctures $p$. Therefore,

$$
\begin{aligned}
\int_{S}\left(\phi_{n} \circ a\right) d a \wedge \lambda & =\lim _{r \rightarrow 0} \sum_{p} \int_{C_{r}(p)} f^{*} \lambda-\int_{S}\left(\psi_{n} \circ a\right) f^{*} d \lambda \\
& \leq \lim _{r \rightarrow 0} \sum_{p} \int_{C_{r}(p)} f^{*} \lambda<C^{\prime}<+\infty .
\end{aligned}
$$

Moreover,

$$
\int_{S}\left(\phi_{n} \circ a\right) d a \wedge \lambda \rightarrow \int_{S}(\phi \circ a) d a \wedge \lambda
$$

as $n \rightarrow \infty$. Hence,

$$
\int_{S}(\phi \circ a) d a \wedge \lambda \leq C^{\prime}
$$


and thus $E(F) \leq \int_{S} d \lambda+C^{\prime}<\infty$. (ii) $\Rightarrow$ (i). First, we obviously have $A(F) \leq E(F)<\infty$. Moreover, $F$ has only positive and negative punctures by assumption, and thus the map $F$ is proper.

The energy and the contact area of a holomorphic map $F:(S \backslash Z, j) \rightarrow(\mathbb{R} \times V, J)$ of finite energy are easily computable in view of Stokes' formula and formula (20). The result is as follows.

Lemma 5.16 Under the condition (i) or (ii) of Lemma 5.15, we denote by $\bar{\gamma}_{1}, \ldots, \bar{\gamma}_{k}$ (resp. $\underline{\gamma}_{1}, \ldots, \underline{\gamma}_{l}$ ) the periodic orbits of $\mathbf{R}$ asymptotic to the positive (resp. negative) punctures of $S$. Then, with $d \lambda=\omega$,

$$
\begin{aligned}
& E_{\omega}(F)=A(F)=\sum_{j=1}^{k} S\left(\bar{\gamma}_{j}\right)-\sum_{j=1}^{l} S\left(\underline{\gamma}_{j}\right) \\
& E_{\lambda}(F)=\sum_{j=1}^{k} S\left(\bar{\gamma}_{j}\right) \\
& E(F)=2 \sum_{j=1}^{k} S\left(\bar{\gamma}_{j}\right)-\sum_{j=1}^{l} S\left(\underline{\gamma}_{j}\right) .
\end{aligned}
$$

\section{Holomorphic curves in almost complex manifolds with cylindrical ends}

\subsection{Energy}

Let $(W, J)$ be an almost complex manifold with cylindrical ends, $W=E_{-} \cup \bar{W} \cup$ $E_{+}$, adjusted to a symplectic form $\omega$ on $\bar{W}$. The diffeomorphism $G^{\delta}: W \rightarrow$ $\stackrel{\circ}{W}=\operatorname{Int} \bar{W}$ defined in Section 3.2 allows us to identify $J$ with the almost complex structure $J^{\delta}=G_{*}^{\delta} J$ on $\stackrel{\circ}{W}$. Both (equivalent) points of view will be useful for us. We will denote by $J_{ \pm}$the cylindrical almost complex structures which are restrictions of $J$ to the ends $E_{ \pm}$, where $E_{-}=(-\infty, 0] \times V_{-}$and $E_{+}=$ $[0, \infty) \times V_{+}$. We need to generalize the definition of energy for a holomorphic curve into an almost complex manifold $(W, J)$ with cylindrical ends, when the structure $J$ is adjusted to a symplectic form $\omega$ on $\bar{W}$. First, we define the $\omega$-energy by the formula

$$
E_{\omega}(F)=\int_{F^{-1}(\bar{W})} F^{*} \omega+\int_{F^{-1}\left(E_{-}\right)} f_{-}^{*} \omega+\int_{F^{-1}\left(E_{+}\right)} f_{+}^{*} \omega,
$$


where $\left.F\right|_{E_{ \pm}}=\left(a_{ \pm}, f_{ \pm}\right)$. Next, we define the $\lambda$-energy $E_{\lambda}(F)$ in a way similar to formula (10) in the case of cylindrical manifolds.

$$
E_{\lambda}(F)=\sup _{\phi_{ \pm} \in \mathcal{C}}\left(\int_{f^{-1}\left(E_{+}\right)}\left(\phi_{+} \circ a_{+}\right) d a_{+} \wedge f^{*} \lambda+\int_{f^{-1}\left(E_{-}\right)}\left(\phi_{-} \circ a_{-}\right) d a_{-} \wedge f^{*} \lambda\right),
$$

where the supremum is taken over all pairs $\left(\phi_{-}, \phi_{+}\right)$from the set $\mathcal{C}$ of all $C^{\infty}$-functions $\phi_{ \pm}: \mathbb{R}_{ \pm} \rightarrow \mathbb{R}_{+}$such that

$$
\int_{0}^{\infty} \phi_{+}(s) d s=\int_{-\infty}^{0} \phi_{-}(s) d s=1 .
$$

Finally, the energy of $F$ is defined as the sum

$$
E(F)=E_{\omega}(F)+E_{\lambda}(F) .
$$

Similarly to Lemma 5.4 for cylindrical manifolds we have

Lemma 6.1 For any holomorphic curve $F$ in $(W, J)$ we have

$$
E_{\omega}(F), E_{\lambda}(F) \geq 0 \text {. }
$$

Moreover,

$$
\int_{f^{-1}\left(E_{+}\right)}\left(\phi_{+} \circ a_{+}\right) d a_{+} \wedge f^{*} \lambda \geq 0
$$

and

$$
\int_{f^{-1}\left(E_{-}\right)}\left(\phi_{-} \circ a_{-}\right) d a_{-} \wedge f^{*} \lambda \geq 0
$$

for all admissible functions $\phi_{ \pm}$.

\subsection{Asymptotic properties of holomorphic curves}

Straightforward extensions of Lemmas 5.5 and 5.6 allow us to describe the asymptotic behavior of $f$ near the punctures of $S$ as follows.

Proposition 6.2 Let $(S, j)$ be a closed Riemann surface, $Z=\left\{z_{1}, \ldots, z_{k}\right\} \subset$ $S$ a set of punctures, and $S^{Z}$ the oriented blow-up at the points of $Z$. Any holomorphic map $F:(S \backslash Z, j) \rightarrow W$ of finite energy and without removable singularities is asymptotically cylindrical near each puncture $z_{i}$ over a periodic 
orbit $\gamma_{i} \in \mathcal{P}=\mathcal{P}_{-} \cup \mathcal{P}_{+}$. The map $F^{\delta}=G^{\delta} \circ F:(S \backslash Z, j) \rightarrow \stackrel{\circ}{W}$ extends to a smooth map $\bar{F}^{\delta}: S^{Z} \rightarrow \bar{W}$, so that the boundary circles $\Gamma_{i}$ are mapped to orbits $\gamma_{i} \in \mathcal{P}$ equivariantly with respect to the canonical action of the circle $\mathbb{R} / \mathbb{Z}$ on $\Gamma_{i}$ and the action of $\mathbb{R} / \mathbb{Z}$ on $\gamma_{i}$ which is generated by the time 1 map of the vector field $T_{i} \mathbf{R}$ if $\gamma_{i} \in \mathcal{P}_{-}$and of $-T_{i} \mathbf{R}$ if $\gamma_{i} \in \mathcal{P}_{+}$, where $T_{i}=S\left(\gamma_{i}\right)=\int_{\gamma_{i}} \lambda .^{2}$

Punctures associated with orbits from $\mathcal{P}_{+}$are called positive, while punctures associated with orbits from $\mathcal{P}_{-}$are called negative. As in the contact cylindrical case, the conditions of finiteness of the full-energy and the $\omega$-energy are essentially equivalent. The following statement is similar to Proposition 5.13.

Proposition 6.3 Let $(W, J)$ be an almost complex manifold with symmetric cylindrical ends adjusted to a symplectic form $\omega$. Suppose that a holomorphic curve with punctures $F:(S \backslash Z, j) \rightarrow(W, J)$ is asymptotic at the positive punctures to the periodic orbits $\bar{\gamma}_{1}, \ldots, \bar{\gamma}_{k} \in \mathcal{P}_{+}$and to the periodic orbits $\underline{\gamma}_{1}, \ldots, \underline{\gamma}_{l} \in \mathcal{P}_{-}$at the negative punctures. Then there exists a positive constant $\bar{C}$ (which depends on $J, \lambda, \omega$ but not $F$ ) such that

$E(F) \leq C\left(\int_{F^{-1}(\bar{W})} F^{*} \omega+\int_{F^{-1}\left(E_{+}\right)} f_{+}^{*} \omega+\int_{F^{-1}\left(E_{-}\right)} f_{-}^{*} \omega\right)+\sum_{1}^{k} S\left(\bar{\gamma}_{i}\right)+\sum_{1}^{l} S\left(\underline{\gamma}_{i}\right)$.

In particular, the energies $E(F)$ are uniformly bounded for all $F$ for which $f$ represents a given homology class in $H_{2}\left(V, \bigcup_{i} \bar{\gamma}_{i} \cup \bigcup_{j} \underline{\gamma}_{j}\right)$.

\section{Holomorphic buildings in cylindrical manifolds $W=\mathbb{R} \times V$}

\subsection{Holomorphic buildings of height 1}

We first introduce in a more systematic way the types of holomorphic curves needed for the compactification of the moduli spaces of holomorphic curves in a cylindrical manifold. Let $(S, j, M \cup Z, D)$ be a nodal Riemann surface, such

\footnotetext{
${ }^{2} \mathrm{~A}$ possibly confusing difference in signs here and in Lemma 5.6 in the cylindrical case is caused by the fact that the action $\mathbb{R} / \mathbb{Z}$ on $\Gamma_{i}$ is defined by the linear complex structure on the plane $T_{z_{i}} S$ tangent to $S$ at the corresponding puncture.
} 
that the set of its marked points is presented as a disjoint union of two ordered sets $M$ and $Z$. The points from $Z$ are called punctures, the points from $M$ are called marked points. The set

$$
D=\left\{\bar{d}_{1}, \underline{d}_{1}, \bar{d}_{2}, \underline{d}_{2}, \ldots, \bar{d}_{s}, \underline{d}_{s}\right\} .
$$

of special marked points is viewed as an unordered set of unordered pairs. The surface $S$ may be disconnected, and the points of any given special pair $\left(\bar{d}_{i}, \underline{d}_{i}\right)$ may belong to the same component, or to different components of $S$. A holomorphic curve $F=(a, f)$ is called the trivial or vertical cylinder if $S$ is the Riemann sphere, the sets $M$ and $D$ are empty, the set $Z$ consists of exactly 2 points and $f$ maps $S$ onto a periodic orbit $\gamma$. A nodal holomorphic curve (or building) of height 1 is a proper holomorphic map

$$
F=(a, f):(S \backslash Z, D, M, j) \rightarrow(\mathbb{R} \times V, J)
$$

of finite energy which sends elements of each special pair to one point:

$$
F\left(\bar{d}_{i}\right)=F\left(\underline{d}_{i}\right) \quad \text { for each } \quad i=1, \ldots, s .
$$

The curve $F$ is called stable in $\mathbb{R} \times V$, if the following conditions are satisfied:

Stab 1 at least one connected component of the curve is not a trivial cylinder,

Stab 2 if $C$ is a connected component of $S$ and the map $\left.F\right|_{C}$ is constant then the Riemann surface $C$ together with all its marked, special marked points and punctures is stable in the sense of Section 4.4 above.

Lemma 5.6 and Proposition 6.2 describe the behavior of a holomorphic curve of height 1 near each puncture. In particular, one can associate a periodic orbit $\gamma_{i} \in \mathcal{P}$ to each puncture $z_{i} \in Z$. The coordinate $a$ of the map $F$ tends near each puncture either to $+\infty$ or $-\infty$. Respectively, we call the punctures positive or negative, and denote the set of positive resp. negative punctures by $\bar{Z}$ resp. $\underline{Z}$. The signature of a holomorphic curve of height 1 is the quadruple of integers $\left(g, \mu, p^{+}, p^{-}\right)$, where $g$ is the arithmetic genus (6) of $S$, where $\mu=\# M$ is the number of marked points, and where $p^{ \pm}$are the numbers of positive respectively negative punctures. As in the case of Riemann surfaces, a holomorphic curve $F$ of height 1 is called connected if the singular Riemann surface $\widehat{S}_{D}$ is connected. Two nodal holomorphic curves,

$$
(F, S, j, M, Z, D) \text { and }\left(F^{\prime}, S^{\prime}, j^{\prime}, M^{\prime}, Z^{\prime}, D^{\prime}\right),
$$

of height 1 are called equivalent if there exists a diffeomorphism $\varphi: S \rightarrow S^{\prime}$ such that

- $\varphi_{*} j=j^{\prime}$ 
- $f^{\prime} \circ \varphi=f, a^{\prime} \circ \varphi=a+$ const

- $\varphi$ sends the ordered sets $M$ and $Z$ isomorphically to $M^{\prime}$ and $Z^{\prime}$.

- $\left.\varphi\right|_{D}$ is an isomorphism $D \rightarrow D^{\prime}$ of unordered sets of unordered pairs.

In particular, we identify curves which differ by a translation along the $\mathbb{R}-$ factor. If the curves $F$ and $F^{\prime}$ are connected, then we can say equivalently that we identify the curves which have the same projections $f$ and $f^{\prime}$ to the contact manifold $V$. The moduli space of stable connected smooth (ie, without double points) holomorphic curves of signature $\left(g, \mu, p^{+}, p^{-}\right)$is denoted by $\mathcal{M}_{g, \mu, p^{+}, p^{-}}(V)$. The bigger moduli space of stable connected nodal holomorphic curves of height 1 and of signature $\left(g, \mu, p^{+}, p^{-}\right)$will be denoted by ${ }^{1} \mathcal{M}_{g, \mu, p^{+}, p^{-}}(V)$. Unlike the case of Riemann surfaces, the space ${ }^{1} \mathcal{M}_{g, \mu, p^{+}, p^{-}}(V)$ is not large enough to compactify $\mathcal{M}_{g, \mu, p^{+}, p^{-}}(V)$. For this purpose we need holomorphic curves (buildings) of height $>1$ discussed in the next section.

\subsection{Holomorphic buildings of height $k$}

Suppose we are given $k$ stable, possibly disconnected nodal curves of height 1 ,

$$
F_{m}=\left(a_{m}, f_{m} ; S_{m}, j_{m}, M_{m}, D_{m}, Z_{m}=\bar{Z}_{m} \cup \underline{Z}_{m}\right), m=1, \ldots, k .
$$

Suppose, in addition, that we are given a cross-ordering $\sigma$ of $M=\bigcup_{m=1}^{k} M_{m}$, which is compatible with the ordering of each individual $M_{i}$, but may mix the points of different $M_{i}$ in an arbitrary way. See Figure 6 , where the ordering of each $M_{i}, i=1,2,3$, is induced by the natural ordering of the index set. Let $\widehat{S}_{m}^{Z_{m}}$ be the circle compactification of the Riemann surface $\left(S_{m}, j_{m}\right)$ at punctures $Z_{m}$, as described in Sections 4.3 and 4.4 above. We denote by $\Gamma_{m}^{+}$and $\Gamma_{m}^{-}$the sets of boundary circles which correspond to the sets $\bar{Z}_{m}$ and $\underline{Z}_{m}$ of punctures. Suppose that for each $m=1, \ldots, k-1$ the number $p_{m}^{+}$of positive punctures of $F_{m}$ is equal to the number $p_{m+1}^{-}$of negative punctures of $F_{m+1}$, and that there is given an orientation reversing diffeomorphism $\Phi_{m}: \Gamma_{m}^{+} \rightarrow \Gamma_{m+1}^{-}$which is an orthogonal map on each boundary component. Using these maps we can, similarly to the construction of the surface $S^{D, r}$ in Section 4.4, form a piecewise smooth surface

$$
S^{Z, \Phi}=S_{1}^{Z_{1}} \underset{\Phi_{1}}{\cup} S_{2}^{Z_{2}} \underset{\Phi_{2}}{\cup} \ldots \underset{\Phi_{k-1}}{\cup} S_{k}^{Z_{k}}
$$

The sequence $F=\left\{F_{1}, \ldots, F_{k}\right\}$ of holomorphic curves of height 1 , together with the decoration maps $\Phi=\left\{\Phi_{1}, \ldots, \Phi_{k-1}\right\}$ and the cross-ordering $\sigma$ is called a holomorphic building of height (or level) $k$, if the compactified maps 


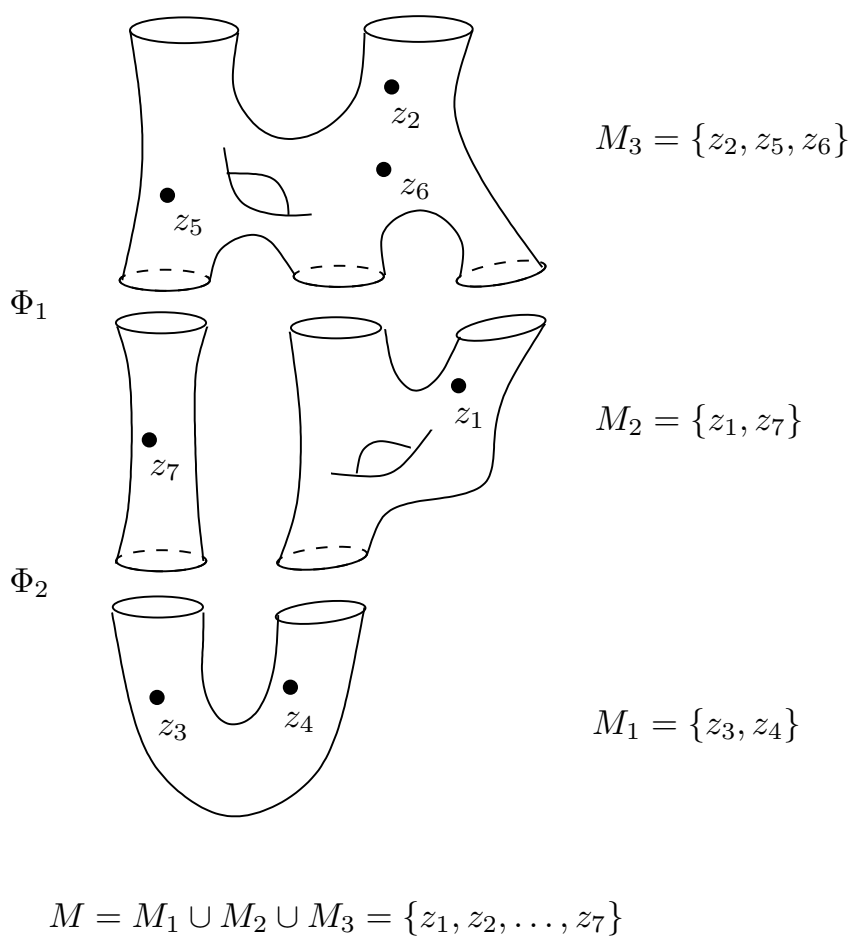

Figure 6: Holomorphic building of height three with an ordered set of marked points

$\bar{f}_{m}: S_{m}^{Z_{m}} \rightarrow V$ fit together into a continuous map $\bar{f}: S^{Z, \Phi} \rightarrow V$. This property implies, in particular, that for $m=1, \ldots, k-1$, the curve $F_{m}$ at its positive punctures is asymptotic to the same periodic orbits as the curve $F_{m+1}$ at its corresponding negative punctures. Two holomorphic buildings of height $k$, namely $(F, \Phi, \sigma)$ and $\left(F^{\prime}, \Phi^{\prime}, \sigma^{\prime}\right)$, where

$$
\begin{aligned}
(F, \Phi, \sigma) & =\left(\left\{F_{1}, F_{2}, \ldots, F_{k}\right\},\left\{\Phi_{1}, \ldots, \Phi_{k-1}\right\}, \sigma\right), \\
F_{i} & =\left(a_{i}, f_{i} ; S_{i},, j_{i}, M_{i}, D_{i}, Z_{i}\right), \text { for } i=1, \ldots, k,
\end{aligned}
$$

and

$$
\begin{aligned}
\left(F^{\prime}, \Phi^{\prime}, \sigma^{\prime}\right) & =\left(\left\{F_{1}^{\prime}, F_{2}^{\prime}, \ldots, F_{k}^{\prime}\right\},\left\{\Phi_{1}^{\prime}, \ldots, \Phi_{k-1}^{\prime}, \sigma^{\prime}\right\}\right), \\
F_{i}^{\prime} & =\left(a_{i}^{\prime}, f_{i}^{\prime} ; S_{i}^{\prime},, j_{i}^{\prime}, M_{i}^{\prime}, D_{i}^{\prime}, Z_{i}^{\prime}\right), \text { for } i=1, \ldots, k,
\end{aligned}
$$

are called equivalent if there exists a sequence $\varphi=\left\{\varphi_{1}, \ldots, \varphi_{k}\right\}$ of diffeomorphisms having the following properties, 
- $\varphi_{m}$, for $m=1, \ldots, k$, is an equivalence between the height 1 holomorphic buildings

$$
\begin{aligned}
& F_{m}=\left(a_{m}, f_{m} ; S_{m}, j_{m}, M_{m}, D_{m}, Z_{m}\right) \text { and } \\
& F_{m}^{\prime}=\left(a_{m}^{\prime}, f_{m}^{\prime} ; S_{m}^{\prime}, j_{m}^{\prime}, M_{m}^{\prime}, D_{m}^{\prime}, Z_{m}^{\prime}\right),
\end{aligned}
$$

- $\varphi$ commutes with the sequences $\Phi$ and $\Phi^{\prime}$ of attaching maps, ie,

$$
\Phi_{m+1}^{\prime} \circ \varphi_{m}=\varphi_{m+1} \circ \Phi_{m}, \text { for } m=1, \ldots, k-1,
$$

- $\varphi_{*} \sigma=\sigma^{\prime}$.

Additionally, we identify holomorphic buildings which differ by a synchronized re-ordering of the pair of the sets $\bar{Z}_{m}$ and $\underline{Z}_{m+1}$ for $1<k \leq m$. To keep the notation simple we will usually drop the cross-ordering $\sigma$ from the notation and will write $(F, \Phi)$ for a holomorphic building of height $k$. The main points to remember are the following. First of all the union of marked points coming from the various levels is ordered. Secondly, the union of the negative punctures on the first level and the positive punctures on the highest level are ordered. Thirdly, there is a compatibility between two consecutive levels in the sense that asymptotic limits match (as specified by the decoration map $\Phi$ ). The genus $g$ of a height $k$ building $(F, \Phi, \sigma)$ is by definition the arithmetic genus of $S^{Z, \Phi}$. Its signature is defined as the quadruple $\left(g, \mu, p^{-}, p^{+}\right)$, where $\mu$ is the total cardinality of the set $M=\bigcup_{1}^{k} M_{i}$ and $p^{+}=p_{k}^{+}$and $p^{-}=p_{1}^{-}$. Note that if $\varphi=\left\{\varphi_{1}, \ldots, \varphi_{k}\right\}$ is an equivalence between $(F, \Phi)$ and $\left(F^{\prime}, \Phi^{\prime}\right)$ then the homeomorphisms $\varphi_{m}: S_{m} \rightarrow S_{m}^{\prime}$, for $m=1, \ldots, k-1$, fit together into a homeomorphism $\bar{\varphi}: S^{Z, \Phi} \rightarrow\left(S^{\prime}\right)^{Z^{\prime}, \Phi^{\prime}}$ between the two surfaces ${ }^{3}$

$$
S^{Z, \Phi}=S_{1}^{Z_{1}} \underset{\Phi_{1}}{\cup} S_{2}^{Z_{2}} \underset{\Phi_{2}}{\cup} \cdots \underset{\Phi_{k-1}}{\cup} S_{k}^{Z_{k}}
$$

and

$$
\left(S^{\prime}\right)^{Z^{\prime}, \Phi^{\prime}}=\left(S_{1}^{\prime}\right)^{Z_{1}^{\prime}} \underset{\Phi_{1}^{\prime}}{\cup}\left(S_{2}^{\prime}\right)^{Z_{2}^{\prime}} \underset{\Phi_{2}^{\prime}}{\cup} \ldots \underset{\Phi_{k-1}^{\prime}}{\cup}\left(S_{k}^{\prime}\right)^{Z_{k}^{\prime}} .
$$

It is also useful to note that in the cases in which all the curves $F_{m}$, for $m=1, \ldots, k$, are connected, or in which they are disconnected but have exactly one component different from a trivial cylinder, one can define the holomorphic building of height $k$ purely in terms of their $V$-components $f_{1}, \ldots, f_{m}$ of the maps $F_{1}, \ldots, F_{k}$. The stability condition for $F$ means the stability of all its components $F_{1}, \ldots, F_{k}$. The moduli space of equivalence classes of stable

\footnotetext{
${ }^{3}$ The converse, however, is not true unless the asymptotic orbits associated with the punctures are simple.
} 


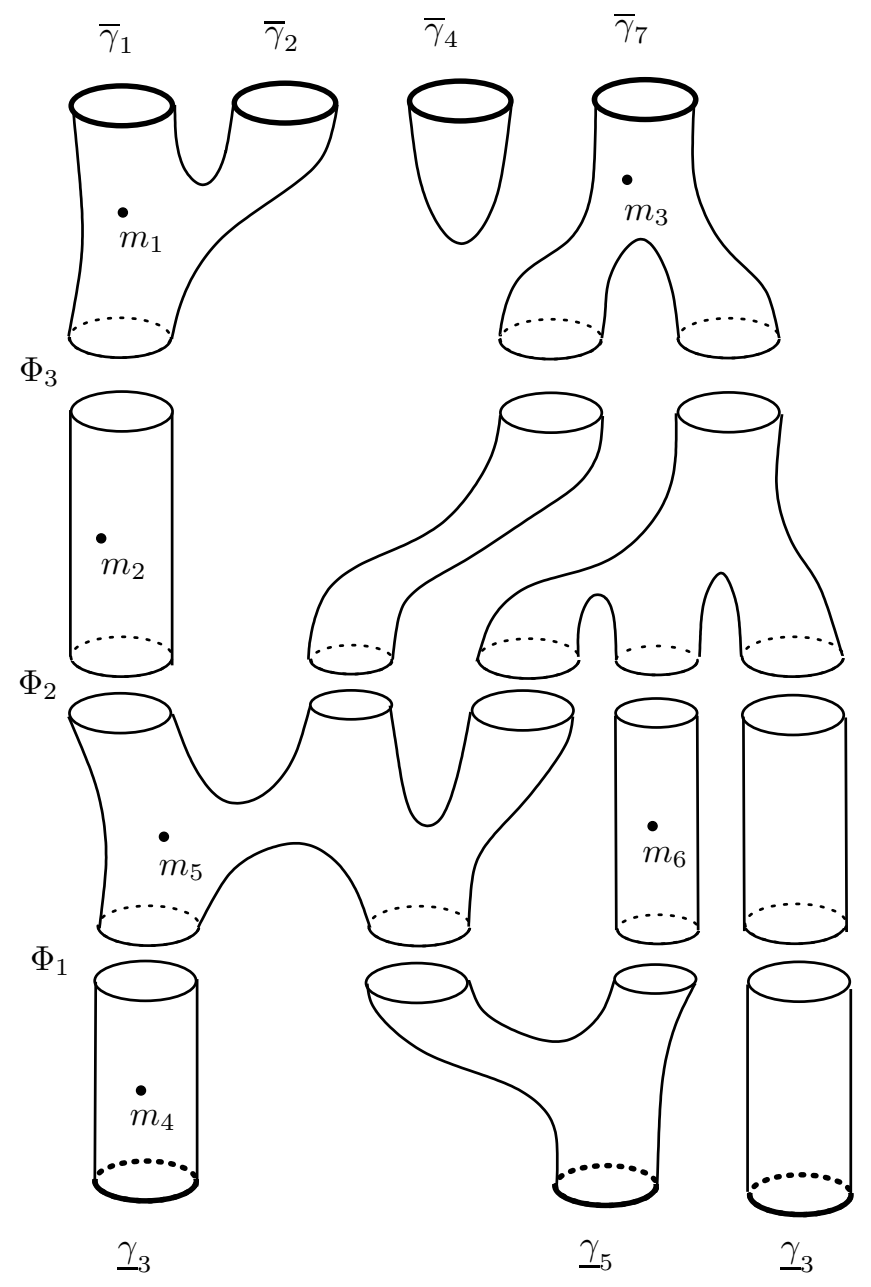

Figure 7: Holomorphic building of height 4

holomorphic buildings of height $k$ and signature $\left(g, \mu, p^{+}, p^{-}\right)$is denoted by ${ }^{k} \mathcal{M}_{g, \mu, p^{-}, p^{+}}(V)$. We set

$$
\overline{\mathcal{M}}_{g, \mu, p^{-}, p^{+}}(V)=\bigcup_{k=1}^{\infty}{ }^{k} \mathcal{M}_{g, \mu, p^{-}, p^{+}}(V)
$$

and

$$
\overline{\mathcal{M}}_{g, \mu}(V)=\bigcup_{p^{-}, p^{+} \geq 0} \overline{\mathcal{M}}_{g, \mu, p^{-}, p^{+}}(V)
$$


With each holomorphic building

$$
\begin{gathered}
(F, \Phi, \sigma)=\left(\left\{F_{1}, F_{2}, \ldots, F_{k}\right\},\left\{\Phi_{1}, \ldots, \Phi_{k-1}\right\}, \sigma\right), \\
F_{m}=\left(a_{m}, f_{m} ; S_{m}, j_{m}, M_{m}, D_{m}, Z_{m}=\bar{Z}_{m} \cup \underline{Z}_{m}\right), \text { for } m=1, \ldots, k,
\end{gathered}
$$

of height $k$ we can associate the underlying nodal Riemann surface

$$
\mathbf{S}_{F}=\left(\bigcup_{1}^{k}\left(S_{m}, j_{m}\right), M=\bigcup_{1}^{k} M_{m} \cup \underline{Z}_{1} \cup \bar{Z}_{k}, D=\bigcup_{1}^{k} D_{m} \cup \bigcup_{1}^{k-1}\left(\bar{Z}_{m} \cup \underline{Z}_{m+1}\right)\right) .
$$

Here we treat the punctures from $\underline{Z}_{1}$ and $\bar{Z}_{k}$ as extra marked points, while the set $\bar{Z}_{1} \cup \underline{Z}_{2} \cup \cdots \cup \bar{Z}_{k-1} \cup \underline{Z}_{k}$ as additional special marked points where the puncture $\bar{z} \in \bar{Z}_{i}$ is coupled with the puncture $\underline{z} \in \underline{Z}_{i+1}, i=1, \ldots, k-1$, if the map $\Phi_{i}$ maps the compactifying circle $\Gamma_{\bar{z}}$ associated with the puncture $\bar{z}$ onto the circle $\Gamma_{\underline{z}}$ associated with $\underline{z}$. The ordering of $M$ is given by $\sigma$, rather than the natural ordering of the union of ordered sets $M_{1}, \ldots, M_{k}$. The maps $\Phi_{i}$ define the decorations at these double points in the sense of Section 4.4 above. Hence, $\mathbf{S}_{F}$ is partially decorated, and in the case when each of the height 1 nodal curves $F_{m}$, for $m=1, \ldots, k$, forming $F$, is equipped with its own decoration $r_{m}$, the Riemann surface $\mathbf{S}_{F}$ gets a full decoration $r_{F, \Phi}=$ $\left\{r_{1}, \ldots, r_{k}, \Phi_{1}, \ldots, \Phi_{k}\right\}$. It is important to realize that the stability of the curve $F$ does not guarantee the stability of the Riemann surface $\mathbf{S}_{F}$. However one can always add a few marked points to some of the sets $M_{i}$ in order to stabilize the Riemann nodal surface $\mathbf{S}^{\prime}=\mathbf{S}_{F^{\prime}}$ which underlies the new holomorphic building $F^{\prime}$.

\subsection{Topology of $\overline{\mathcal{M}}_{g, \mu, p_{-}, p_{+}}(V)$}

The notion of convergence in $\overline{\mathcal{M}}_{g, \mu, p_{-}, p_{+}}(V)$ which we define below is compatible with the metric space structure on $\overline{\mathcal{M}}_{g, \mu, p_{-}, p_{+}}(V)$ defined in Appendix B.2. In particular, the topology introduced here is Hausdorff. Suppose that we are given a sequence

$$
\left(F_{i}, \Phi_{i}\right) \in \overline{\mathcal{M}}_{g, \mu, p_{-}, p_{+}}(V), \text { for } i \geq 1,
$$

of holomorphic buildings of height $\leq k$. The sequence $\left(F_{i}, \Phi_{i}\right)$ converges to a building $(F, \Phi) \in \overline{\mathcal{M}}_{g, \mu, p_{-}, p_{+}}(V)$ of height $k$ if there exist sequences $M_{i}^{\prime}$ of extra sets of marked points for the buildings $\left(F_{i}, \Phi_{i}\right)$ and a set $M^{\prime}$ of extra marked points for the building $(F, \Phi)$, which have the same cardinality $N$ and which stabilize the corresponding underlying Riemann surfaces, and such that the following conditions are satisfied. Let

$$
\left(\mathbf{S}_{F_{i}}, r_{F_{i}, \Phi_{i}}\right)=\left(S_{i}, j_{i}, M_{i} \cup M_{i}^{\prime}, D_{i}, r_{i}\right)
$$


and

$$
\left(\mathbf{S}_{F}, r_{F, \Phi}\right)=\left(S, j, M \cup M^{\prime}, D, r\right)
$$

be the decorated stable nodal Riemann surfaces underlying the curves

$$
\left(F_{m}, \Phi_{m}\right) \text { and }(F, \Phi)
$$

with extra marked points. Then there exist diffeomorphisms $\varphi_{i}: S^{D, r} \rightarrow S^{D_{i}, r_{i}}$ with $\varphi_{i}(M)=M_{i}$ and $\varphi_{i}\left(M^{\prime}\right)=M_{i}^{\prime}$ which satisfy the conditions CRS1-CRS3 in the definition of convergence of Riemann surfaces and, in addition, the following conditions.

CHC1 The sequence of the compactified projections $\bar{f}_{i} \circ \varphi_{i}: S^{D, r} \rightarrow V$ converges to $\bar{f}: S^{D, r} \rightarrow V$ uniformly.

CHC2 Let us denote by $C_{l}$ the union of components of $S^{D, r} \backslash \bigcup \Gamma_{m}$ which correspond to the same level $l=1, \ldots, k$ of the building $F$. Then there exist sequences of real numbers $c_{i}^{l}$, for $l=1, \ldots, k$ and $i \geq 1$, such that $\left.\left(a_{i} \circ \varphi_{i}-a-c_{i}^{l}\right)\right|_{C_{l}} \rightarrow 0$ in the $C_{\mathrm{loc}}^{0}-$ topology.

The $C_{\mathrm{loc}}^{0}$-convergence in $\mathrm{CHC} 2$ can be equivalently replaced by the $C_{\mathrm{loc}}^{\infty}-$ convergence in view of the elliptic regularity theory.

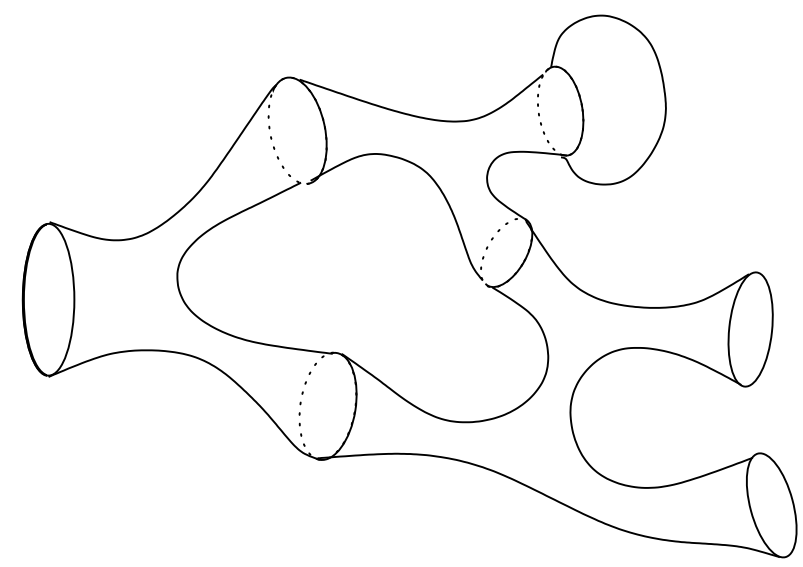

Figure 8: The map $\bar{f}: S^{D, r} \rightarrow V$ 


\section{Holomorphic buildings in manifolds with cylindri- cal ends}

\subsection{Holomorphic buildings of height $k_{-}|1| k_{+}$}

We now generalize the notion of a holomorphic building to the case, in which the target manifold has cylindrical ends, rather than being cylindrical. A nodal holomorphic curve in $W$, or a holomorphic building of height 1 is defined similarly to a nodal curve of height 1 in a cylindrical manifold, ie, it is a proper holomorphic map

$$
F=(S \backslash Z, j, D, M) \rightarrow(W, J)
$$

of finite energy which sends elements of every special pair to one point, ie, $F\left(\bar{d}_{i}\right)=F\left(\underline{d}_{i}\right)$ for $i=1, \ldots, s$. Suppose that we are given:

(i) A holomorphic building of height $k_{+}$in the cylindrical manifold $\mathbb{R} \times V_{+}$:

$$
\begin{gathered}
\left(F^{+}, \Phi^{+}\right)=\left(\left\{F_{1}, F_{2}, \ldots, F_{k_{+}}\right\},\left\{\Phi_{1}, \ldots, \Phi_{k_{+}-1}\right\}\right) \\
F_{i}=\left(a_{i}, f_{i} ; S_{i}, j_{i}, M_{i}, D_{i}, \underline{Z}_{i} \cup \bar{Z}_{i}\right), \text { for } i=1, \ldots, k_{+} .
\end{gathered}
$$

(ii) A holomorphic building of height $k_{-}$in the cylindrical manifold $\mathbb{R} \times V_{-}$:

$$
\begin{gathered}
\left(F^{-}, \Phi^{-}\right)=\left(\left\{F_{-k_{-}}, F_{-k_{-}+1}, \ldots, F_{-1}\right\},\left\{\Phi_{-k_{-}}, \ldots, \Phi_{-2}\right\}\right) \\
F_{i}=\left(a_{i}, f_{i} ; S_{i}, j_{i}, M_{i}, D_{i}, \underline{Z}_{i} \cup \bar{Z}_{i}\right), \text { for } i=-k_{-}, \ldots,-1 .
\end{gathered}
$$

(iii) A nodal holomorphic curve $\left(F_{0}, S_{0}, D_{0}, M_{0}, \underline{Z}_{0} \cup \bar{Z}_{0}, j_{0}\right)$ in $(W, J)$. We denote by $\Gamma_{0}^{ \pm}$the sets of boundary circles which correspond to the punctures $\underline{Z}_{0}$ and $\bar{Z}_{0}$.

(iv) An ordering of $\bigcup_{k_{-}}^{k_{+}} M_{i}$ which is compatible with the ordering of each individual $M_{i}$ but not necessarily respecting the numbering of the sets $M_{k_{-}}, \ldots, M_{k_{+}}$.

Suppose that

- the number $p_{0}^{+}$of positive punctures of $F_{0}$ is equal to the number $p_{1}^{-}$of negative punctures of $F_{1}$,

- the number $p_{-1}^{+}$of positive punctures of $F_{-1}$ is equal to the number $p_{0}^{-}$ of negative punctures of $F_{0}$,

- for $m=-1,0$ there is given an orientation reversing diffeomorphism $\Phi_{m}: \Gamma_{m}^{+} \rightarrow \Gamma_{m+1}^{-}$which is orthogonal on each boundary component. 


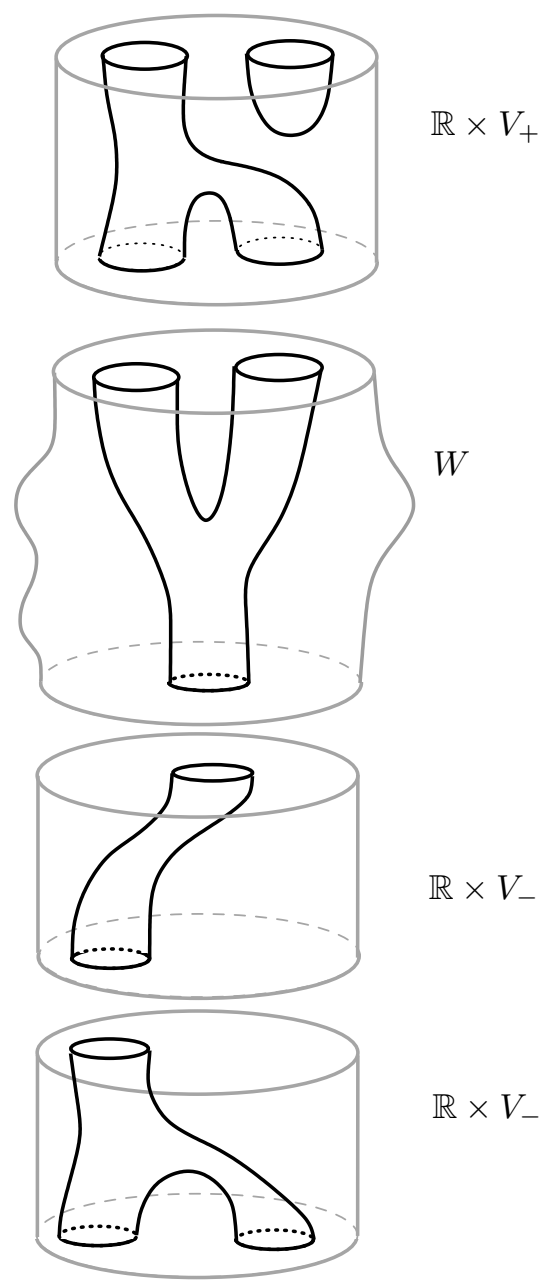

Figure 9: Holomorphic building of height 2|1|1

Let $S^{0}=S_{0}^{Z_{0}}$ be the oriented blow-up of $S_{0}$ at the punctures from $Z_{0}$, and let the surfaces

$$
\begin{aligned}
& S^{+}=S^{Z^{+}, \Phi^{+}}=S_{1}^{Z_{1}} \underset{\Phi_{1}}{\cup} S_{2}^{Z_{2}} \underset{\Phi_{2}}{\cup} \ldots \underset{\Phi_{k_{+}-1}}{\cup} S_{k_{+}}^{Z_{k_{+}}} \\
& S^{-}=S^{Z^{-}, \Phi^{-}}=S_{-k_{-}}^{Z_{-k_{-}}} \underset{\Phi_{-k_{-}}}{\cup} S_{-k_{-}+1}^{Z_{-k_{-}+1}} \underset{\Phi_{-k_{-}+1}}{\cup} \ldots \underset{\Phi_{-2}}{\cup} S_{-1}^{Z_{-1}}
\end{aligned}
$$

Geometry $8 \mathcal{T}$ Topology, Volume 7 (2003) 
be defined as in Section 7.2. Gluing $S^{-}$and $S^{0}$ by means of $\Phi_{-1}$, and $S^{0}$ and $S^{+}$by means of $\Phi_{0}$ we obtain the piecewise-smooth surface

$$
\bar{S}=S^{-} \underset{\Phi_{-1}}{\cup} S^{0} \underset{\Phi_{0}}{\cup} S^{+} .
$$

The last condition in the definition of a building in $W$ of height $k_{-}|1| k_{+}$can now be formulated as follows:

(v) for a sufficiently small $\delta>0$ the maps

$$
\bar{f}^{-}: S^{-} \rightarrow V_{-}, \quad G^{\delta} \circ F^{0}: S^{0} \rightarrow \stackrel{\circ}{W}, \quad \text { and } \bar{f}^{+}: S^{+} \rightarrow V_{+}
$$

fit together into a continuous map $\bar{F}: \bar{S} \rightarrow \bar{W}$.

We will also say sometimes that a holomorphic building of height $k_{-}|1| k_{+}$consists of 3 layers, namely the lower layer is a holomorphic building $F_{-}$of height $k_{-}$, the main layer is a holomorphic curve $F_{0}$ ( of height 1 ), and the upper layer is a holomorphic building $F_{+}$of height $k_{+}$. The equivalence relation for holomorphic buildings of height $k_{-}|1| k_{+}$is defined similarly to buildings in cylindrical manifolds except that there is no translation to be quotient out in the central layer. As in the cylindrical case the genus $g$ of the building $(F, \varphi)$

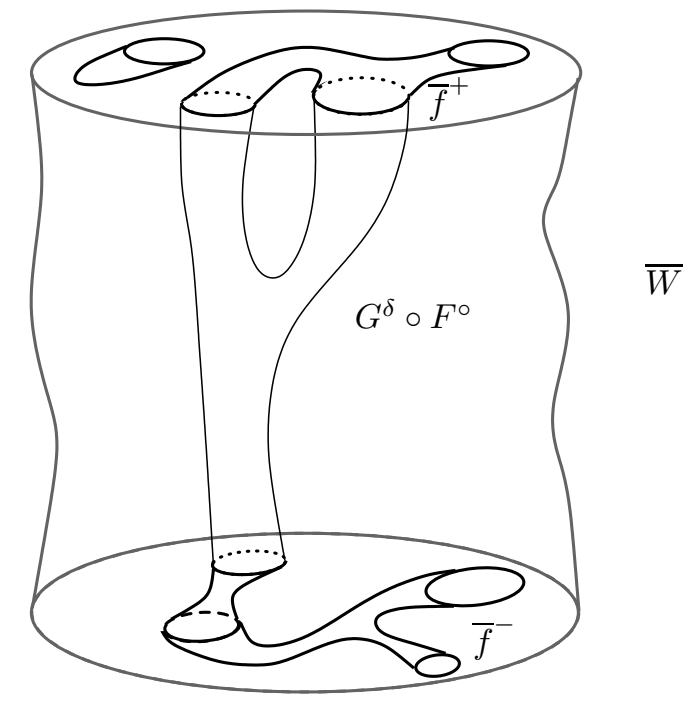

Figure 10: The maps $\bar{f}^{-}, G^{\delta} \circ F^{\circ}$ and $\bar{f}^{+}$for a continuous map $\bar{F}: \bar{S} \rightarrow \bar{W}$

of height $k_{-}|1| k_{+}$is by definition the arithmetic genus of $\bar{S}$. Its signature is defined as the quadruple $\left(g, \mu, p^{-}, p^{+}\right)$, where $\mu$ is the total cardinality of the 
set $M=\bigcup_{k_{-}}^{k_{+}} M_{i}$, and where $p^{+}=p_{k_{+}}^{+}$and $p^{-}=p_{-k_{-}}^{-}$. The energies $E(F)$, $E_{\lambda}(F)$ and $E_{\omega}(F)$ of a curve $F$ of height $k_{-}|1| k_{+}$are defined by the formulas

$$
\begin{aligned}
E_{\omega}(F) & =\sum_{-k_{-}}^{k_{+}} E_{\omega}\left(F_{i}\right) \\
E_{\lambda}(F) & =\max _{-k_{-} \leq i \leq k_{+}} E_{\lambda}\left(F_{i}\right) \\
E(F) & =E_{\lambda}(F)+E_{\omega}(F) .
\end{aligned}
$$

A holomorphic building $\left(F^{-}, F^{0}, F^{+}\right)$of height $k_{+}|1| k_{-}$is called stable if all its three layers,

- the height $k_{+}$building in $\mathbb{R} \times V_{+}$,

- the height $k_{-}$building $F^{-}$in $\mathbb{R} \times V_{-}$and

- the curve $F^{0}$ in $W$,

are stable. Here the map $F_{0}$ into $W$ is called stable if for every component $C$ of the underlying Riemann surface $S_{0}$ either the restriction $\left.F_{0}\right|_{C}$ is non constant or in case $\left.F_{0}\right|_{C}$ is constant, then $C$, equipped with all its marked points from $D_{0} \cup M_{0}$ and all its punctures from $Z_{0}$, is stable. Equivalently, this requires that the automorphism group of every component of $S_{0}$ equipped with all its distinguished points, is finite. The moduli spaces of holomorphic buildings of signature $\left(g, \mu, p^{-}, p^{+}\right)$and height $k_{-}|1| k_{+}$in $(W, J)$ is denoted by $k_{-}, k_{+} \mathcal{M}_{g, \mu, p^{-}, p^{+}}(W, J)$. We set

$$
\begin{aligned}
& k_{-}, k_{+} \mathcal{M}_{g, \mu}(W, J)=\bigcup_{p^{-}, p^{+} \geq 0} k_{-}, k_{+} \mathcal{M}_{g, \mu, p^{-}, p^{+}}(W, J) \\
& \overline{\mathcal{M}}_{g, \mu, p^{-}, p^{+}}(W, J)=\bigcup_{k_{-}, k_{+} \geq 0} k_{-}, k_{+} \mathcal{M}_{g, \mu, p^{-}, p^{+}}(W, J) \\
& \overline{\mathcal{M}}_{g, \mu}(W, J)=\bigcup_{p^{-}, p^{+} \geq 0} \overline{\mathcal{M}}_{g, \mu, p^{-}, p^{+}}(W, J) .
\end{aligned}
$$

\subsection{Topology of $\overline{\mathcal{M}}_{g, \mu}(W, J)$}

In this section we will spell out the meaning of convergence of a sequence of smooth curves

$$
F^{(k)} \in \mathcal{M}_{g, \mu}(W, J)={ }^{k_{-}, k_{+}} \mathcal{M}_{g, \mu}(W, J), \quad \text { with } \quad k_{-}=0=k_{+},
$$

for $k \geq 1$, to a building

$$
F=\left(\left\{F_{-k_{-}}, \ldots, F_{0}, \ldots, F_{k_{+}}\right\} ;\left\{\Phi_{-k_{-}}, \ldots, \Phi_{0}, \ldots, \Phi_{k_{+}}\right\}\right)
$$


from ${ }^{k_{-}, k_{+}} \mathcal{M}_{g, \mu, p^{-}, p^{+}}(W, J)$. A more general definition of convergence in the case in which $F^{(k)}$ is a sequence of holomorphic buildings from

$$
k_{-}, k_{+} \overline{\mathcal{M}}_{g, \mu, p^{-}, p^{+}}(W, J)=\bigcup_{\substack{0 \leq i \leq k_{-} \\ 0 \leq j \leq k_{+}}} i, j \mathcal{M}_{g, \mu, p^{-}, p^{+}}(W, J)
$$

is similar and left to the reader. The sequence $F^{(k)}$ converges to $F$ if there exist sequences $M^{(k)}$ of extra sets of marked points for the curves $F^{(k)}$ and a set $M$ of extra marked points for the building $F$ which have the same cardinality $N$ and which stabilize the corresponding underlying Riemann surfaces such that the following conditions are satisfied. Let $\mathbf{S}_{k}=\left(S^{(k)}, j^{(k)}, M^{(k)}\right)$ be Riemann surfaces underlying $F^{(k)}$ with the extra marked points $M^{(k)}$, and $(\mathbf{S}, \Phi)$ be the decorated Riemann surface underlying the building $F$ with the extra set $M$ of marked points. We consider, as in (26), the surface

$$
\bar{S}=S^{-} \underset{\Phi_{-1}}{\cup} S^{0} \underset{\Phi_{0}}{\cup} S^{+}=S_{-k_{-}}^{Z_{-k_{-}}} \underset{\Phi_{-k_{-}}}{\cup} S_{-k_{-}+1}^{Z_{-k_{-}+1}} \underset{\Phi_{-k_{-}+1}}{\cup} \cdots \underset{\Phi_{k_{+}-1}}{\cup} S_{k_{+}}^{Z_{k_{+}}} .
$$

with a conformal structure $j$ which is degenerate along the union $\Gamma$ of special circles. Let $\bar{F}: \bar{S} \rightarrow \bar{W}$ be the map described in part (v) of the definition of a holomorphic building of height $k_{-}|1| k_{+}$. We also abbreviate

$$
\dot{S}_{i}:=(\bar{S} \backslash \Gamma) \cap S_{i} \text { for } i=-k_{-}, \ldots, k_{+} .
$$

Suppose that there exists a sequence of diffeomorphisms $\varphi_{k}: \bar{S} \rightarrow S^{(k)}$ which satisfies the conditions CRS1-CRS3 in the definition of the convergence of decorated Riemann surfaces in section 4.5, and require, in addition, the following conditions.

CHCE1 For sufficiently large $k \geq K$, the images $\left.F^{(k)} \circ \varphi_{k}\right|_{\dot{S}_{i}}$ for $i=$ $-k_{-}, \ldots,-1$, are contained in the cylindrical end $E_{-}$, and the the images $\left.F^{(k)} \circ \varphi_{k}\right|_{\dot{S}_{i}}$ for $i=1, \ldots, k_{+}$, are contained in the cylindrical end $E_{+}$of the manifold $W$.

CHCE2 There exist constants $c_{i}^{(k)}$ for $i=-k_{-}, \ldots,-1,1, \ldots, k_{+}$and $k \geq$ $K$, such that $\left.\widetilde{F}_{i}^{(k)} \circ \varphi_{k}\right|_{\dot{S}_{i}}$ converge to $F_{i}$ uniformly on compact sets, where $F_{i}^{(k)}=\left(a_{i}^{(k)}, f_{i}^{(k)}\right)$ and $\widetilde{F}_{i}^{(k)}=\left(a_{i}^{(k)}+c_{i}^{(k)}, f_{i}^{(k)}\right)$.

CHCE3 The sequence $G^{\delta} \circ F^{(k)} \circ \varphi_{k}: \bar{S} \rightarrow \bar{W}$ converges uniformly to $\bar{F}$.

Note that the space $\overline{\mathcal{M}}_{g, \mu}(W, J)$ can be metrized similar to the way it is done in Appendix B.2 below for the moduli spaces of holomorphic buildings in cylindrical manifolds. For different values of $p^{ \pm}$the spaces $\overline{\mathcal{M}}_{g, \mu, p^{-}, p^{+}}(W, J)$ are disjoint open-closed subsets of $\overline{\mathcal{M}}_{g, \mu}(W, J)$. 


\section{Holomorphic buildings in split almost complex manifolds}

\subsection{Holomorphic buildings of height $\underset{1}{\stackrel{k_{0}}{V}}$}

Let us recall the splitting construction from Section 3.4. We begin with a closed almost complex manifold $(W, J)$, cut it open along a co-oriented hypersurface $V$ to get a manifold $\stackrel{\circ}{W}$ with two new boundary components $V^{\prime}, V^{\prime \prime}$ diffeomorphic to $V$, and attach to $V^{\prime}$ and $V^{\prime \prime}$ cylindrical ends, thus obtaining a manifold with cylindrical ends of the form

$$
\widetilde{W}=(-\infty, 0] \times V \underset{0 \times V=V^{\prime}}{\cup} \stackrel{\circ}{W} \underset{V^{\prime \prime}=0 \times V}{\cup}[0,+\infty) \times V .
$$

Of course, the manifold $\widetilde{W}$ is diffeomorphic to $W \backslash V$. The almost complex structure $J$ canonically extends to $\widetilde{W}$ as an almost complex structure $\widetilde{J}$ which is translation invariant on the ends. A holomorphic building $(F, \Phi)$ of height $\underset{1}{k_{0}}$ in the split manifold $(\widetilde{W}, \widetilde{J})$ is determined by the following data:

(i) a height 1 holomorphic curve (building) $F_{0}$ in $(\widetilde{W}, \widetilde{J})$;

(ii) a height $k_{0}$ holomorphic building

$$
\begin{gathered}
\left(F^{\prime}, \Phi^{\prime}\right)=\left(\left\{F_{1}, \ldots, F_{k_{0}}\right\},\left\{\Phi_{1}, \ldots, \Phi_{k_{0}-1}\right\}\right), \\
F_{i}=\left(a_{i}, f_{i} ; S_{i}, j_{i}, M_{i}, D_{i}, \underline{Z}_{i} \cup \bar{Z}_{i}\right), \text { for } i=1, \ldots, k_{0},
\end{gathered}
$$

in $(\mathbb{R} \times V, J)$;

(iii) orientation reversing diffeomorphisms $\Phi_{0}: \Gamma_{0}^{+} \rightarrow \Gamma_{1}^{-}$and $\Phi_{k_{0}}: \Gamma_{k_{0}}^{+} \rightarrow$ $\Gamma_{0}^{-}$, orthogonal on each boundary component;

(iv) an ordering of $\bigcup_{0}^{k_{0}} M_{i}$ which is compatible with the ordering of each individual $M_{i}$ but not necessarily respecting the numbering of the sets $M_{0}, \ldots, M_{k_{0}}$.

Using blow-up at the punctures and identification of the boundary components by means of the mappings $\Phi$, we define the following surfaces:

$$
\begin{aligned}
S^{0} & =S_{0}^{Z_{0}} \\
S^{\prime} & =S_{1}^{Z_{1}} \underset{\Phi_{1}}{\cup} S_{2}^{Z_{2}} \underset{\Phi_{2}}{\cup} \ldots \underset{\Phi_{k_{0}-1}}{\cup} S_{k_{0}}^{Z_{k_{0}}} \\
\bar{S} & =S^{\prime} \underset{\Phi_{0}, \Phi_{k_{0}}}{\cup} S^{0} .
\end{aligned}
$$

Geometry $8 \mathcal{T}$ Topology, Volume 7 (2003) 


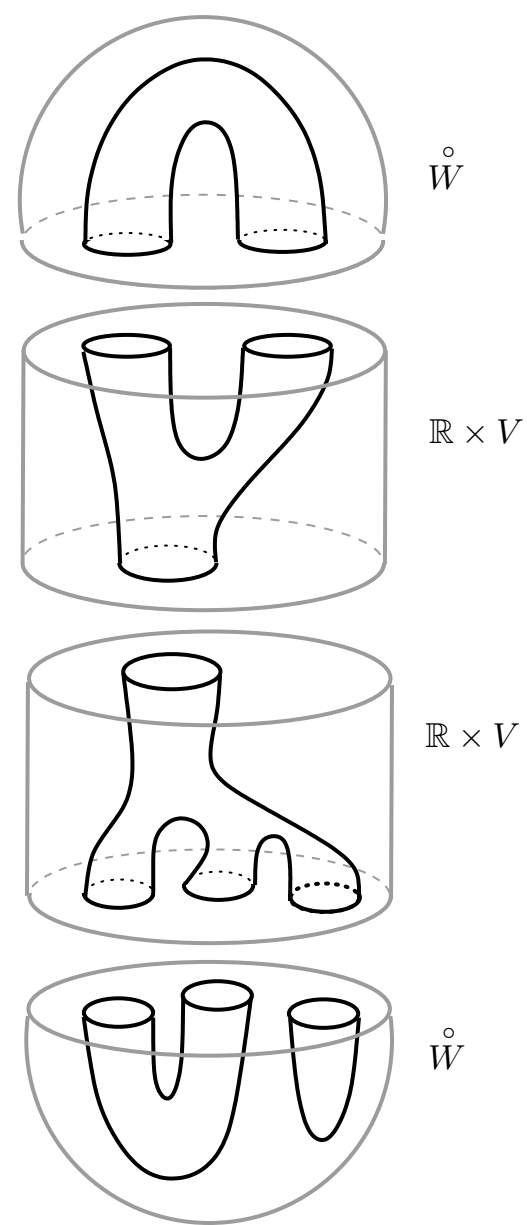

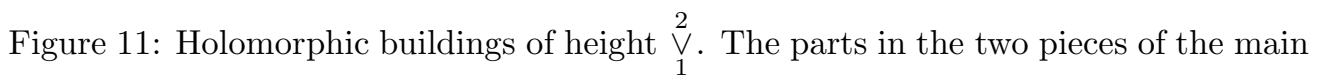
layer are parameterized by $S_{0}$, the two levels of the insert layer are parameterized by $S_{1}$ and $S_{2}$

The last requirement in the definition of the holomorphic building of height $\underset{1}{k_{0}}$ is the following:

(v) the maps $\overline{f^{\prime}}: S^{\prime} \rightarrow V$ and $G^{\delta} \circ F_{0}: S_{0} \rightarrow \stackrel{\circ}{W}$ fit together into a continuous map $\bar{F}: \bar{S} \rightarrow W$.

The holomorphic building has two layers: the main layer $F_{0}$, and the insert layer $\left(F^{\prime}, \Phi^{\prime}\right)$. The equivalence relation for holomorphic buildings of height $k_{-}|1| k_{+}$is defined similarly to buildings in cylindrical manifolds except that 
there is no translation to be quotient out in the insert layer. The holomorphic building $(F, \Phi)$ is said to be stable if all its layers are stable. The moduli spaces of holomorphic buildings of signature $(g, \mu)$ and height ${\underset{1}{V}}_{1}^{k_{0}}$ in the split manifold $(\widetilde{W}, \widetilde{J})$ is denoted by ${ }^{k_{0}} \mathcal{M}_{g, \mu}(\widetilde{W}, \widetilde{J})$. We set, recalling section 3.4,

$$
\begin{aligned}
\overline{\mathcal{M}}_{g, \mu}(\widetilde{W}, \widetilde{J}) & =\bigcup_{k_{0} \geq 0}{ }^{k_{0}} \mathcal{M}_{g, \mu}(\widetilde{W}, \widetilde{J}) \\
\mathcal{M}_{g, \mu}\left(W^{[0, \infty)}, J^{[0, \infty)}\right) & =\bigcup_{\tau \in[0, \infty)} \overline{\mathcal{M}}_{g, \mu}\left(W^{\tau}, J^{\tau}\right) \\
\overline{\mathcal{M}}_{g, \mu}\left(W^{[0, \infty]}, J^{[0, \infty]}\right) & =\mathcal{M}_{g, \mu}\left(W^{[0, \infty)}, J^{[0, \infty)}\right) \cup \overline{\mathcal{M}}_{g, \mu}(\widetilde{W}, \widetilde{J}) .
\end{aligned}
$$

The space $\overline{\mathcal{M}}_{g, \mu}\left(W^{[0, \infty]}, J^{[0, \infty]}\right)$ can be topologized by introducing a metric similar to the way it is done in Appendix B.2 for the case of holomorphic buildings in cylindrical manifolds. The formula for the distance between a holomorphic curve $(F, \Phi)$ in $\left(W^{\tau}, J^{\tau}\right)$ and a holomorphic curve $\left(F^{\prime}, \Phi^{\prime}\right)$ in $\left(W^{\tau^{\prime}}, J^{\tau^{\prime}}\right)$ must contain the additional term $\left|\frac{1}{1+\tau}-\frac{1}{1+\tau^{\prime}}\right|$. Let us spell out the meaning of the convergence in this topology, of a sequence $F^{(k)}$ of stable holomorphic curves into a sequence of almost complex manifolds $\left(W^{k}, J^{k}\right)$ degenerating into the split almost complex manifold $(\widetilde{W}, \widetilde{J})$. We say that a sequence of stable holomorphic curves $F^{(k)}$ into $\left(W^{k}, J^{k}\right)$ converges to a stable level $k_{0}$ holomorphic

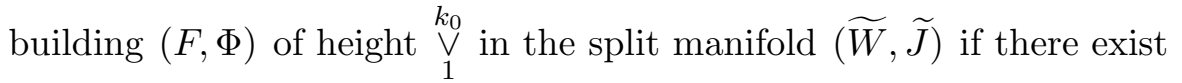

- extra sets of marked points $M^{(k)}$ and $M$ of the same cardinality, which stabilize the Riemann surfaces which underly the holomorphic curves $F^{(k)}$ and the holomorphic building $(F, \Phi)$,

- a sequence of diffeomorphisms $\varphi_{k}: \bar{S} \rightarrow S^{(k)}$,

- sequences $c_{i}^{(k)} \in \mathbb{R}$, for $i=1, \ldots, k_{0}$,

such that the conditions CRS1-CRS3 of the Deligne-Mumford convergence in Section 4.5 are satisfied and such that, in addition, the following conditions are met,

CHCS1 $\left.F_{0}^{(k)} \circ \varphi_{k}\right|_{\dot{S}_{0}}$ converges to $F_{0}$ uniformly on compact sets,

CHCS2 for sufficiently large $k \geq K$, the images $\left.F^{(k)} \circ \varphi_{k}\right|_{\dot{S}_{i}}$ for $i=1, \ldots, k_{0}$, are contained in the cylindrical portion $[-k, k] \times V$ of $W^{k}$,

CHCS3 $\left.\left(c_{i}^{(k)}+a_{k} \circ \varphi_{k}, f_{k} \circ \varphi_{k}\right)\right|_{\dot{S}_{i}}$ converge uniformly on compact sets to $F_{i}$ for $i=1, \ldots, k_{0}$. 


\subsection{Energy bounds for holomorphic curves in the process of splitting}

Suppose now that $W$ is endowed with a symplectic structure $\omega$ compatible with $J$, and that the splitting along $V$ is adjusted to $\omega$. The energy, and $\omega$-energy of a holomorphic building of height ${\underset{1}{V}}_{1}^{k_{0}}$ in a split almost complex manifold is naturally defined by the formulas

$$
\begin{aligned}
E_{\omega}(F) & =E_{\omega}\left(F_{0}\right)+E_{\omega}\left(F^{\prime}\right) \\
E_{\lambda}(F) & =\max \left(E_{\lambda}\left(F_{0}\right), E_{\lambda}\left(F^{\prime}\right)\right) \\
E(F) & =E_{\lambda}(F)+E_{\omega}(F) .
\end{aligned}
$$

So far we never specified the symplectic forms on the family of almost complex manifolds $\left(W^{\tau}, J^{\tau}\right)$ converging to the split manifold $(\widetilde{W}, \widetilde{J})$. This can be done but only in such a way that in the limit the symplectic structure either degenerates or blows up. Instead, we slightly modify the notion of energies for holomorphic curves in $\left(W^{\tau}, J^{\tau}\right)$. Let us recall that

$$
W^{\tau}=\stackrel{\circ}{W} \cup I_{\tau},
$$

where $I_{\tau}=[-\tau, \tau] \times V$. Given a holomorphic curve $F:(S, j) \rightarrow\left(W^{\tau}, J^{\tau}\right)$ we define its $\omega$-energy as

$$
E_{\omega}(F)=\int_{\substack{\circ \\ F^{-1}(W)}} F^{*} \omega+\int_{F^{-1}\left(I_{\tau}\right)} F^{*} p_{V}^{*} \omega,
$$

where $p_{V}$ is the projection $I_{\tau}=[-\tau, \tau] \times V \rightarrow V$. We also define

$$
E_{\lambda}(F)=\sup \int_{F(S) \cap I_{\tau}}\left(\phi \circ p_{\mathbb{R}} \circ F\right) d t \wedge \lambda,
$$

where $p_{\mathbb{R}}$ is the projection $I_{\tau}=[-\tau, \tau] \times V \rightarrow[-\tau, \tau]$, and the supremum is taken over all function $\phi:[-\tau, \tau] \rightarrow \mathbb{R}_{+}$with $\int_{[-\tau, \tau]} \phi(t) d t=1$. Finally, we set

$$
E(F)=E_{\lambda}(F)+E_{\omega}(F) .
$$

With these definitions we immediately get

Lemma 9.1 Given a sequence of holomorphic curves $F^{(k)}$ in $\left(W^{k}, J^{k}\right)$ which converges to a holomorphic building $F$ in the split manifold $(\widetilde{W}, \widetilde{J})$, then

$$
\lim _{k \rightarrow \infty} E_{\omega}\left(F^{(k)}\right)=E_{\omega}(F) .
$$


It turns out that a uniform bound on the $\omega$-energy automatically implies a uniform bound on the full energy.

Lemma 9.2 There exists a constant $C$ which depends only on $(W, J), V$ and $\lambda$ such that for every $\tau>0$ and every holomorphic curve $F:(S, j) \rightarrow\left(W^{\tau}, J^{\tau}\right)$,

$$
E(F) \leq C E_{\omega}(F) .
$$

Proof Let us denote $V_{+}=V \times \tau \subset W^{\tau}=\stackrel{\circ}{W} \cup[-\tau, \tau] \times V$. We will show that there exists a constant $K$ such that for any $\tau>0$ and any holomorphic curve $F:(S, j) \rightarrow\left(W^{\tau}, J^{\tau}\right)$ we have

$$
\left|\int_{F^{-1}\left(V_{+}\right)} \lambda\right| \leq K E_{\omega}(F)
$$

Take a sufficiently small tubular neighborhood $U_{\varepsilon}=V_{+} \times[0,1]$ of $V_{+}$inside $\stackrel{\circ}{W}$, so that $V_{+}=V_{+} \times 0$, and pull-back $\lambda$ to $U_{\varepsilon}$ via the projection $V_{+} \times[0, \varepsilon] \rightarrow$ $V_{+}=V$. Let $t \in[0,1]$ denote a coordinate in $U_{\varepsilon}$ which corresponds to the second factor and $V_{+}^{\prime}=V_{+} \times 1$. Applying Stokes' theorem we find

$$
\begin{aligned}
\int_{F^{-1}\left(U_{\varepsilon}\right)} F^{*} d(t \lambda) & =\int_{F^{-1}\left(V_{+}^{\prime}\right)} F^{*} \lambda, \\
\int_{F^{-1}\left(U_{\varepsilon}\right)} F^{*} d \lambda & =\int_{F^{-1}\left(V_{+}^{\prime}\right)} F^{*} \lambda-\int_{F^{-1}\left(V_{+}\right)} F^{*} \lambda .
\end{aligned}
$$

On the other hand, taking into account that $F$ is $J$-holomorphic and that $\omega$ is compatible with $J$ on $\stackrel{\circ}{W}$, we have

$$
\begin{gathered}
\left|\int_{F^{-1}\left(U_{\varepsilon}\right)} F^{*} d(t \lambda)\right| \leq K_{1} \int_{F^{-1}\left(U_{\varepsilon}\right)} F^{*} \omega, \\
\left|\int_{F^{-1}\left(U_{\varepsilon}\right)} F^{*} d \lambda\right| \leq K_{2} \int_{F^{-1}\left(U_{\varepsilon}\right)} F^{*} \omega .
\end{gathered}
$$

Consequently,

$$
\left|\int_{F^{-1}\left(V_{+}\right)} F^{*} \lambda\right| \leq\left(K_{1}+K_{2}\right) \int_{F^{-1}\left(U_{\varepsilon}\right)} F^{*} \omega \leq K E_{\omega}(F) .
$$

Similarly, with the obvious notation,

$$
\left|\int_{F^{-1}\left(V_{-}\right)} F^{*} \lambda\right| \leq K E_{\omega}(F)
$$


The rest of the proof follows the lines of Proposition 5.13.

\section{Compactness theorems}

\subsection{Statement of main theorems}

In this section we prove the main results of the paper.

Theorem 10.1 Let $(\mathbb{R} \times V, J)$ be a symmetric cylindrical almost complex manifold. Suppose that the almost complex structure $J$ is adjusted to the taming symplectic form $\omega$. Then for every $E>0$, the space $\overline{\mathcal{M}}_{g, \mu}(V) \cap\{E(F) \leq$ $E\}$ is compact.

Theorem 10.2 Let $\left(W=E_{-} \cup \bar{W} \cup E_{+}, J\right)$ be an almost complex manifold with symmetric cylindrical ends. Suppose that $J$ is adjusted to a symplectic form $\omega$ on $W$. Then for every $E>0$, the space $\overline{\mathcal{M}}_{g, \mu}(W, J) \cap\{E(F) \leq E\}$ is compact.

Theorem 10.3 Let $(\widetilde{W}, \widetilde{J})$ be a split almost complex manifold which is obtained, as in Section 3.4 above, by splitting a closed almost complex manifold $(W, J)$ along a co-oriented hypersurface $V$. Suppose that $J$ is compatible with a symplectic form $\omega$ on $W$, and $\left(\left.\widetilde{J}\right|_{\mathbb{R} \times V},\left.\omega\right|_{\mathbb{R} \times V}\right)$ satisfy the symmetry condition from Section 2.1. Then for every $E>0$, the space $\overline{\mathcal{M}}_{g, \mu}\left(W^{[0, \infty]}, J^{[0, \infty]}\right) \cap$ $\{E(F) \leq E\}$ is compact.

First note that in all three cases it is enough to prove the sequential compactness because the corresponding moduli spaces $\overline{\mathcal{M}}_{g, \mu}(V)$ are metric spaces. Next, it is enough to consider sequences of curves of height $k=1$. Indeed, we can handle each level separately. Moreover, we can assume all these curves to be smooth, ie, having no double points $D$, because the double points can be treated as extra marked points. Finally, the energy bound and the Morse-Bott condition guarantee that there are only finitely many possibilities for the asymptotics at the punctures, Hence it is enough to prove the following three theorems.

Theorem 10.4 Let

$$
\begin{gathered}
\mathbf{F}_{n}=\left(F_{n}=\left(a_{n}, f_{n}\right) ; S_{n}, j_{n}, M_{n}, \underline{Z}_{n} \cup \bar{Z}_{n}\right), \\
\bar{Z}_{n}=\left\{\left(\bar{z}_{1}\right)_{n}, \ldots,\left(\bar{z}_{p^{+}}\right)_{n}\right\}, \underline{Z}_{n}=\left\{\left(\underline{z}_{1}\right)_{n}, \ldots,\left(\underline{z}_{p^{-}}\right)_{n}\right\},
\end{gathered}
$$


be a sequence of smooth holomorphic curves in $(W=\mathbb{R} \times V, J)$ of the same signature $\left(g, \mu, p^{-}, p^{+}\right)$and which are asymptotic at the corresponding punctures to orbits from the same component of the space of periodic orbits $\mathcal{P}$. Then there exists a subsequence that converges to a stable holomorphic building $F$ of height $k$.

Theorem 10.5 Let

$$
\begin{gathered}
\mathbf{F}_{n}=\left(F_{n} ; S_{n}, j_{n}, M_{n}, \underline{Z}_{n} \cup \bar{Z}_{n}\right), \\
\bar{Z}_{n}=\left\{\left(\bar{z}_{1}\right)_{n}, \ldots,\left(\bar{z}_{p^{+}}\right)_{n}\right\}, \underline{Z}_{n}=\left\{\left(\underline{z}_{1}\right)_{n}, \ldots,\left(\underline{z}_{p^{-}}\right)_{n}\right\},
\end{gathered}
$$

be a sequence of smooth holomorphic curves in $(W, J)$ of the same signature $\left(g, \mu, p^{-}, p^{+}\right)$and which are asymptotic at the corresponding punctures to orbits from the same component of the space of periodic orbits $\mathcal{P}$. Then there exists a subsequence that converges to a stable holomorphic building $\mathbf{F}$ of height $k_{-}|1| k_{+}$.

Theorem 10.6 Let

$$
\begin{gathered}
\mathbf{F}_{n}=\left(F_{n} ; S_{n}, j_{n}, M_{n}, \underline{Z}_{n} \cup \bar{Z}_{n}\right), \\
\bar{Z}_{n}=\left\{\left(\bar{z}_{1}\right)_{n}, \ldots,\left(\bar{z}_{p^{+}}\right)_{n}\right\}, \underline{Z}_{n}=\left\{\left(\underline{z}_{1}\right)_{n}, \ldots,\left(\underline{z}_{p^{-}}\right)_{n}\right\},
\end{gathered}
$$

be a sequence of smooth holomorphic curves in manifolds $\left(W^{n}, J^{n}\right)$ converging to a split manifold $(\widetilde{W}, \widetilde{J})$. Suppose all the curves have the same signature $\left(g, \mu, p^{-}, p^{+}\right)$and are asymptotic at the corresponding punctures to orbits from the same components of the space of periodic orbits $\mathcal{P}$. Then there exists a

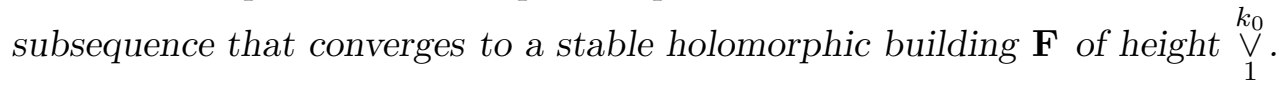

\subsection{Proof of Theorems 10.4, 10.5 and 10.6}

The proofs of all three theorems are very similar, though differ in some details. In each of the four steps of the proof we first discuss in detail the cylindrical case, and then indicate which changes, if any, are necessary for the two other theorems. 


\subsubsection{Step 1: Gradient bounds}

First, we observe that there is a bound $N=N(E)$, depending only on the energy of the curve, on the number of marked points which should be added to stabilize the underlying surfaces $\mathbf{S}_{n}^{F}$. Hence, by adding $N$ extra marked points we can assume that all the surfaces $\mathbf{S}_{n}=\mathbf{S}_{n}^{F}=\left(S_{n}, j_{n}, M_{n}, \underline{Z}_{n} \cup \bar{Z}_{n}\right)$ are stable. Using Theorem 4.2 we may assume, by passing to a subsequence, that the sequence of Riemann surfaces $\mathbf{S}_{n}$ converges to a decorated Riemann nodal surface

$$
\mathbf{S}=\{S, j, M, D, \bar{Z} \cup \underline{Z}, r\} .
$$

Next, we are going to add more marked points to obtain gradient bounds on the resulting punctured surfaces. First, we do it in the cylindrical case. We continue to write $\rho(x)=\operatorname{injrad}(x)$ for the injectivity radius.

Lemma 10.7 There exists an integer $K=K(E)$ which depends only on the energy bound $E$ such that, by adding to each marked point set $M_{n}$ a disjoint set

$$
Y_{n}=\left\{y_{n}^{(1)}, u_{n}^{(1)}, \ldots, y_{n}^{(K)}, u_{n}^{(K)}\right\} \subset \dot{S}_{n}=S_{n} \backslash\left(M_{n} \cup \underline{Z}_{n} \cup \bar{Z}_{n}\right)
$$

of cardinality $2 K$, we can arrange a uniform gradient bound

$$
\left\|\nabla F_{n}(x)\right\| \leq \frac{C}{\rho(x)}, x \in \dot{S}_{n} \backslash Y_{n}
$$

where the gradients are computed with respect to the cylindrical metric on $\mathbb{R} \times V$ associated with a fixed Riemannian metric on $V$, and the hyperbolic metric on $\dot{S}_{n} \backslash Y_{n}$, and where $\rho(x)$ is the injectivity radius of this hyperbolic metric at the point $x \in \dot{S}_{n} \backslash Y_{n}$.

Proof Suppose we are given a sequence of points $x_{n}^{(1)} \in \dot{S}_{n}$ which satisfies the property

$$
\lim _{n \rightarrow \infty} \rho\left(x_{n}^{(1)}\right)\left\|\nabla F_{n}\left(x_{n}^{(1)}\right)\right\| \rightarrow \infty .
$$

By translating the maps $F_{n}=\left(a_{n}, f_{n}\right)$ along the $\mathbb{R}$-factor of $\mathbb{R} \times V$ we can arrange that $a_{n}\left(x_{n}^{(1)}\right)=0$ for all $n$. There exist (injective) holomorphic charts $\psi_{n}: D \rightarrow \mathcal{D}_{n} \subset \dot{S}_{n}$ with $\psi_{n}(0)=x_{n}^{(1)}$ and with

$$
C_{1} \rho\left(x_{n}^{(1)}\right) \leq\left\|\nabla \psi_{n}\right\| \leq C_{2} \rho\left(x_{n}^{(1)}\right)
$$

for two positive constants $C_{1}, C_{2}$. This can easily been seen by taking fundamental domains in the hyperbolic upper half-plane uniformizing components of the thin part of $\dot{S}_{n}$. Then we have $\left\|\nabla\left(F_{n} \circ \psi_{n}\right)(0)\right\| \rightarrow \infty$ as $n \rightarrow \infty$ and 
hence, using Lemma 5.11 we conclude that there exist sequences $y_{n}^{(1)} \rightarrow 0$ and $c_{n}, R_{n} \rightarrow \infty$ as $n \rightarrow \infty$ such that the rescaled maps

$$
\widetilde{F}_{n}: D_{R_{n}} \rightarrow(W, J): z \mapsto F_{n} \circ \tilde{\psi}_{n}(z),
$$

where

$$
\tilde{\psi}_{n}(z):=\psi_{n}\left(y_{n}^{(1)}+c_{n}^{-1} z\right),
$$

converge to a holomorphic map $\widetilde{F}_{\infty}$ satisfying $E\left(\widetilde{F}_{\infty}\right) \leq C$ and $E_{\omega}\left(\widetilde{F}_{\infty}\right)>\hbar$. Here $D_{R}$ denotes the disc $\{|z|<R\} \subset \mathbb{C}$. Moreover, this map is either a holomorphic sphere or a holomorphic plane $\mathbb{C}$ asymptotic as $|z| \rightarrow \infty$ to a closed $\mathbf{R}$-orbit. Let us choose a sequence $u_{n}^{(1)}=y_{n}^{(1)}+c_{n}^{-1} \in D$ and set $\bar{y}_{n}^{(1)}=\psi_{n}\left(y_{n}^{(1)}\right), \bar{u}_{n}^{(1)}=\psi_{n}\left(u_{n}^{(1)}\right)$. Then $\bar{y}_{n}^{(1)}$ and $\bar{u}_{n}^{(1)}$ are distinct points in $\psi_{n}\left(D_{R_{n}}\right) \subset \mathcal{D}_{n}$, and $\operatorname{dist}_{n}\left(\bar{y}_{n}^{(1)}, \bar{u}_{n}^{(1)}\right) \underset{n \rightarrow \infty}{\rightarrow} 0$, where $\operatorname{dist}_{n}$ is the distance function on $\dot{S}_{n}$ defined by the hyperbolic metric $h^{j_{n}, M_{n} \cup \underline{Z}_{n} \cup \bar{Z}_{n}}$. Thus according to Proposition 4.3 (a subsequence of) the sequence of marked Riemann surfaces $\mathbf{S}_{n}^{(1)}=\left(S_{n}, j_{n}, M_{n} \cup\left\{\bar{y}_{n}^{(1)}, \bar{u}_{n}^{(1)}\right\}, \underline{Z}_{n} \cup \bar{Z}_{n}\right)$ converges to a nodal decorated Riemann surface $\mathbf{S}^{(2)}$ obtained from $\mathbf{S}^{(1)}=\mathbf{S}$ by adding one or two spherical components. Figure 12 illustrates a possible scenario, while all possible cases are illustrated by Figure 5 . Note that by construction exactly one of these components contain the marked points $\bar{y}^{(1)}$ and $\bar{u}^{(1)}$, which correspond to the sequences $\bar{y}_{n}^{(1)}$. This bubble serves as the domain of the map $\widetilde{F}_{\infty}$, and thus have the $\omega$-energy concentration for large $n$ exceeding $\hbar$. Set now

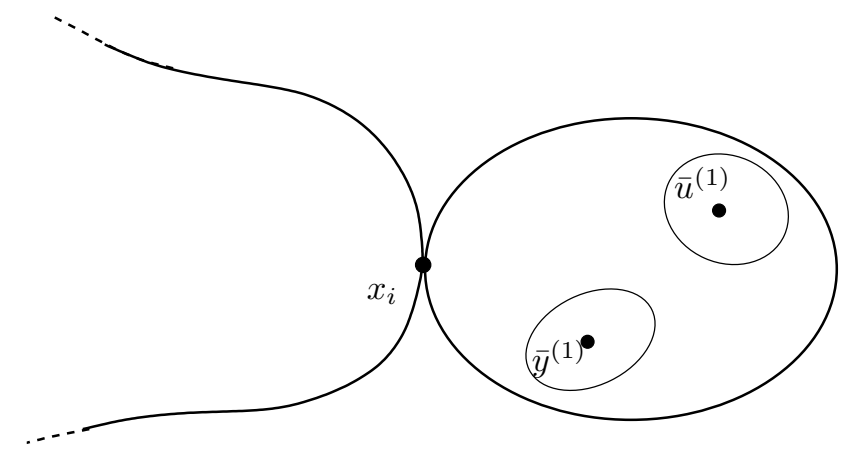

Figure 12: The surface $\mathbf{S}^{(2)}$ a spherical bubble containing the points $\bar{y}^{(1)}$ and $\bar{u}^{(1)}$

$M_{n}^{(1)}=M_{n} \cup\left\{y^{(1)}, u^{(1)}\right\}$ and repeat the above analysis for the sequence of holomorphic curves

$$
\mathbf{F}_{n}^{(2)}=\left(F_{n}=\left(a_{n}, f_{n}\right) ; S_{n}, j_{n}, M^{(1)}, \underline{Z}_{n} \cup \bar{Z}_{n}\right) .
$$


If the inequality (32) does not hold for the hyperbolic metric on

$$
\dot{S}^{(2)}=\dot{S}_{n} \backslash\left\{y_{n}^{(1)}, u_{n}^{(1)}\right\}
$$

then we repeat the above bubbling-off analysis, thus constructing:

- a sequence of points $x_{n}^{(2)} \in \dot{S}_{n}^{(2)}$ having the property

$$
\lim _{n \rightarrow \infty} \rho\left(x_{n}^{(2)}\right)\left\|\nabla F_{n}\left(x_{n}^{(2)}\right)\right\| \rightarrow \infty .
$$

- holomorphic charts $\psi_{n}^{(2)}: D \rightarrow \mathcal{D}_{n}^{(2)} \subset \dot{S}_{n}$ satisfying $\psi_{n}^{(2)}(0)=x_{n}^{(2)}$ and

$$
C_{1}^{(2)} \rho\left(x_{n}^{(2)}\right) \leq\left\|\nabla \psi_{n}^{(2)}\right\| \leq C_{2}^{(2)} \rho\left(x_{n}^{(2)}\right),
$$

for two positive constants $C_{1}^{(2)}, C_{2}^{(2)}$.

- sequences $y_{n}^{(2)} \rightarrow 0$ and $c_{n}^{(2)}, R_{n}^{(2)} \rightarrow \infty$ as $n \rightarrow \infty$ such that the rescaled maps

$$
\begin{aligned}
\widetilde{F}_{n}^{(2)}: D_{R_{n}^{(2)}} & \rightarrow(W, J), \\
z & \mapsto F_{n}^{(2)} \circ \widetilde{\psi}_{n}^{(2)}(z),
\end{aligned}
$$

where

$$
\widetilde{\psi}_{n}^{(2)}(z)=\psi_{n}^{(2)}\left(y_{n}^{(2)}+\frac{z}{c_{n}^{(2)}}\right)
$$

converge to a holomorphic map $\widetilde{F}_{\infty}^{(2)}$ satisfying

$$
E\left(\widetilde{F}_{\infty}^{(2)}\right) \leq C \text { and } E_{\omega}\left(\widetilde{F}_{\infty}^{(2)}\right)>\hbar .
$$

Notice that there exist sequences $K_{n}^{(1)}, K_{n}^{(2)} \underset{n \rightarrow \infty}{\rightarrow} \infty$ satisfying

$$
K_{n}^{(1)}<R_{n} \text { and } K_{n}^{(2)}<R_{n}^{(2)},
$$

such that the discs $\tilde{\psi}_{n}\left(D_{K_{n}^{(1)}}\right)$ and $\tilde{\psi}_{n}^{(2)}\left(D_{K_{n}^{(2)}}\right)$ do not intersect. Indeed, for any fixed $K>0$ the gradients $\left\|\nabla F_{n}\right\|$ are uniformly bounded on $\psi_{n}\left(D_{K}\right)$ and go to $\infty$ as $n \rightarrow \infty$ on $\psi_{n}^{(2)}\left(D_{K}\right)$. Set

$$
u_{n}^{(2)}=y_{n}^{(2)}+\frac{1}{c_{n}^{(2)}}, \quad \bar{y}_{n}^{(2)}=\psi_{n}^{(2)}\left(y_{n}^{(2)}\right), \quad \bar{u}_{n}^{(2)}=\psi_{n}^{(2)}\left(u_{n}^{(2)}\right) .
$$

Then the sequence

$$
\mathbf{F}_{n}^{(3)}=\left(F_{n}=\left(a_{n}, f_{n}\right) ; S_{n}, j_{n}, M^{(2)}=M_{n}^{(1)} \cup\left\{y_{n}^{(2)}, u_{n}^{(2)}\right\}, \underline{Z}_{n} \cup \bar{Z}_{n}\right) .
$$

has in the limit an extra bubble $\widetilde{F}_{\infty}^{(2)}$ disjoint from $\widetilde{F}_{\infty}^{(1)}=\widetilde{F}_{\infty}$ whose energy satisfies $E_{\omega}\left(\widetilde{F}_{\infty}^{(2)}\right)>\hbar$. Hence, the uniform bound on the $\omega$-energy guarantees that, after adding finitely many pairs of marked points

$$
y_{n}^{(1)}, u_{n}^{(1)}, \ldots, y_{n}^{(K)}, u_{n}^{(K)},
$$




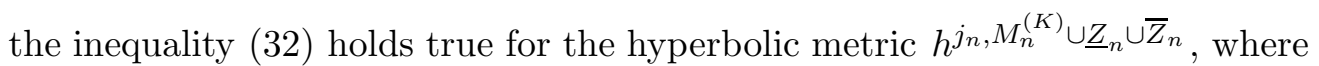
$M_{n}^{(K)}=M_{n} \cup\left\{y_{n}^{(1)}, u_{n}^{(1)}, \ldots, y_{n}^{(K)}, u_{n}^{(K)}\right\}$. The proof of Lemma is complete.

\section{Case of manifolds with cylindrical ends and the splitting case}

Lemma 10.7 has obvious analogues in these two cases. In the case of mappings to a manifold $W$ with cylindrical ends the gradients should be computed with respect to a fixed metric on $W$ which is cylindrical at the ends. In the splitting case the gradients are computed with respect to a sequence of metrics on the target manifold which arise in the process of splitting. These metrics have longer and longer cylindrical inserts. The proof works without any serious changes, except that one needs to analyze separately the case in which the sequence $F_{n}\left(x_{n}\right)$ stays in a compact subset of $W$ and the case in which (a subsequence of) it is contained in the cylindrical part. In the second case the proof is identical while in the first case it does not make sense, and there is no need to shift the map in order to fix its $\mathbb{R}$-component.

\subsubsection{Step 2: Convergence of Riemann surfaces and convergence away from nodes}

This step is common in all three theorems.

We will assume from now on that we added enough extra marked points to stabilize the underlying surfaces $\mathbf{S}_{n}^{F}$ and to ensure the gradient bounds (32). Then using Theorem 4.2 we may assume, by passing to a subsequence, that the sequence of Riemann surfaces $\mathbf{S}_{F_{n}}$ converges to a decorated Riemann nodal surface

$$
\mathbf{S}=\{S, j, M, D, \bar{Z} \cup \underline{Z}, r\} .
$$

From section 4.5 we recall that in our situation, where $D_{n}=\emptyset$ and where $M_{n} \cup Z_{n}$ and $M \cup Z$ in abuse of notation are again denoted by $M_{n}$ and $M$, this means the following. There exists a sequence of diffeomorphisms

$$
\varphi_{n}: S^{D, r} \rightarrow S_{n} \text { with } \varphi_{n}(M)=M_{n}
$$

having the following properties.

- There exist disjoint closed geodesics $\Gamma_{i}^{n}$ for $i=1, \ldots, k$ on $S_{n} \backslash M_{n}$ with respect to the hyperbolic metrics $h^{j_{n}, M_{n}}$, for all $n \geq 1$, such that $\Gamma_{i}:=\varphi_{n}^{-1}\left(\Gamma_{i}^{n}\right)$ are special circles on $S^{D, r} \backslash M$.

- $\varphi_{n}^{*} j_{n} \rightarrow j$ in $C_{\mathrm{loc}}^{\infty}\left(S^{D, r} \backslash \bigcup_{1}^{k} \Gamma_{i}\right)$ 
- Given a component $C$ of $\operatorname{Thin}_{\varepsilon}(\mathbf{S}) \subset \dot{S}^{D, r}$ which contains a special circle $\Gamma_{i}$ and given a point $c_{i} \in \Gamma_{i}$, we consider for every $n \geq 1$ the geodesic arc $\delta_{i}^{n}$ for the induced metric $h_{n}=\varphi_{n}^{*} h^{j_{n}, M_{n}}$ which intersects $\Gamma_{i}$ orthogonally at the point $c_{i}$, and whose ends are contained in the $\varepsilon$-thick part of the metric $h_{n}$. Then $\left(C \cap \delta_{i}^{n}\right)$ converges as $n \rightarrow \infty$ in $C^{0}$ to a continuous geodesic for the metric $h^{\mathrm{S}}$ which passes through the point $c_{i}$.

In addition, according to Lemma 10.7 we may assume that

$$
\left\|\nabla\left(F_{n} \circ \varphi_{n}\right)(x)\right\| \leq \frac{C}{\rho(x)}, x \in S \backslash \bigcup \Gamma_{i} .
$$

These gradient bounds allow to apply locally the Gromov-Schwarz Lemma 5.1 to conclude uniform bounds for all derivatives of $F_{n} \circ \varphi_{n}$ on the $\varepsilon$-thick part on $\operatorname{Thick}_{\varepsilon}(\mathbf{S})$ for every $\varepsilon>0$, and therefore Ascoli-Arzela's theorem allows us to extract a subsequence converging in $C_{\text {loc }}^{\infty}$ on $S \backslash \bigcup \Gamma_{i}=\bigcup_{\varepsilon} \operatorname{Thick}_{\varepsilon}(\mathbf{S}){ }^{4}$

\subsubsection{Step 3: Convergence in the thin part}

Cylindrical case Let us denote by $C_{1}, \ldots, C_{N}$ the connected components of $S \backslash \bigcup \Gamma_{i}$. We already may assume that the holomorphic maps $F_{n} \circ \varphi_{n}$ converge on each component $C_{i}$ for $i=1, \ldots, N$. Our next goal is to understand the asymptotic behavior of the limit map $F=(a, f)$ on the component $C_{i}$ near a node. First, if $F$ is bounded near the node, then, by the removable singularity theorem, Lemma 5.5, the map $F=(a, f)$ extends continuously on $C_{i}$ across the node. On the other hand, if $F$ is unbounded near the node, the behavior of $F$ is described by Proposition 5.6. Namely, there exists a closed R-orbit $\gamma \in \mathcal{P}$ such that the map $F$ is asymptotic to $\gamma$ near the node either at the positive or at the negative end. Moreover, the map $f$ extends continuously to the circle at infinity which compactifies the puncture.

Behavior near a node adjacent to two components Given a node of $S$ adjacent to the two components $C_{i}$ and $C_{j}$, the asymptotic behavior of $F$ on the two components might be different at first sight. For example, $F$ could be asymptotic to different $\mathbf{R}$-orbits, or $F$ could be asymptotic to an $\mathbf{R}$-orbit on $C_{i}$ and could converge to a point on $C_{j}$, or it could converge to different points. Even if $F$ is asymptotic to the same orbit on $C_{i}$ and $C_{j}$ we still have to worry

\footnotetext{
${ }^{4}$ Let us recall that the convergence in cylindrical manifolds is defined up to translation along the $\mathbb{R}$-factor. In particular, when the surface $S \backslash \bigcup \Gamma_{i}$ is disconnected one may need to shift the maps of the sequence restricted to different components by different constants.
} 
about a loss of $\omega$-energy (which may happen only in the non-contact case), and a possible shift in the asymptotic parameterizations of the orbits. To each node adjacent to two components, we can associate two asymptotic limits $\gamma^{+}$ and $\gamma^{-}$, one for each component of $S \backslash \bigcup \Gamma_{i}$ adjacent to the node. Each $\gamma^{ \pm}$is either a point or a periodic orbit from $\mathcal{P}$. The node in question appeared as a

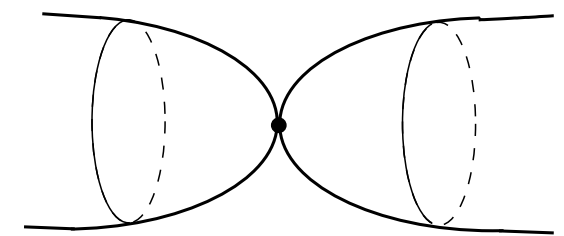

Figure 13: Node adjacent to two components

result of the degeneration of a component of the $\varepsilon$-thin part of $S_{n}$. In other words, there exists a component $T_{n}^{\varepsilon}$ of the $\varepsilon$-thin part of the hyperbolic metric $h^{n}=\varphi_{n}^{*} h^{j_{n}, M_{n}}$ on $S=S^{D, r}$, with conformal parametrization

$$
g_{n}^{\varepsilon}: A_{n}^{\varepsilon}=\left[-N_{n}^{\varepsilon}, N_{n}^{\varepsilon}\right] \times S^{1} \rightarrow\left(T_{n}^{\varepsilon}, j_{n}\right),
$$

such that in the $C^{\infty}\left(S^{1}\right)$-sense,

$$
\left.\lim _{\varepsilon \rightarrow 0} \lim _{n \rightarrow \infty} f_{n} \circ \varphi_{n} \circ g_{n}^{\varepsilon}\right|_{\left( \pm N_{n}^{\varepsilon}\right) \times S^{1}}=\gamma^{ \pm},
$$

where $\gamma^{ \pm}$are either two periodic orbits of $\mathbf{R}$ or two points of $V$. Moreover, for any constant $K$ we have

$$
\left.\lim _{n \rightarrow \infty} f_{n} \circ \varphi_{n} \circ g_{n}^{\varepsilon}\right|_{\left( \pm N_{n}^{\varepsilon} \mp K\right) \times S^{1}}=\gamma^{ \pm} .
$$

Remark 10.8 It is possible that both orbits $\gamma^{+}$and $\gamma^{-}$may appear at the same end of the cylindrical manifold. In this case one of the orbits must have an opposite orientation. For the following discussion the orientation of $\gamma^{ \pm}$will be irrelevant, and thus it will not be specified it in our notation.

Note that the parameterizations $g_{n}^{\varepsilon}$ can be chosen so that they satisfy the gradient bounds

$$
\left\|\nabla g_{n}^{\varepsilon}(x)\right\| \leq C \rho\left(g_{n}^{\varepsilon}(x)\right),
$$

where the gradients are computed with respect to the flat metric in the source and the hyperbolic metric in the target, while the injectivity radius is computed 
with respect to the hyperbolic metric. Together with the estimate (32) this implies a uniform (ie, independent of $n$ and $\varepsilon$ ) gradient bound

$$
\sup _{x \in A_{n}^{\varepsilon}}\left\|\nabla\left(F_{n} \circ \varphi_{n} \circ g_{n}^{\varepsilon}(x)\right)\right\| \leq C .
$$

Given a sequence $\varepsilon_{k} \rightarrow 0$ let us choose a subsequence $\varepsilon_{k_{n}} \rightarrow 0$ such that

$$
\left.\lim _{n \rightarrow \infty} F_{k_{n}} \circ \varphi_{k_{n}} \circ g_{k_{n}}^{\varepsilon_{k_{n}}}\right|_{ \pm N_{k_{n}}^{\varepsilon_{k_{n}}} \times S^{1}}=\gamma^{ \pm},
$$

and introduce the abbreviated notation $\widehat{N}_{n}=N_{k_{n}}^{\varepsilon_{k_{n}}}, \widehat{g}_{n}=\varphi_{k_{n}} \circ g_{k_{n}}^{\varepsilon_{k_{n}}}, \widehat{f}_{n}=$ $f_{k_{n}} \circ \widehat{g}_{k_{n}}$ and $\widehat{F}_{n}=F_{k_{n}} \circ \widehat{g}_{k_{n}}$, so that we get

$$
\lim _{n \rightarrow \infty} \widehat{f}_{n}\left( \pm \widehat{N}_{n} \times S^{1}\right)=\gamma^{ \pm}
$$

For large $n$, the loops $\left.\widehat{f}_{n}\right|_{\left( \pm \widehat{N}_{n}\right) \times S^{1}}$ are sufficiently $C^{\infty}\left(S^{1}\right)-=$ close to $\gamma^{ \pm}$and hence the cylinder $\left.\widehat{f}_{n}\right|_{\left[-\widehat{N}_{n}, \widehat{N}_{n}\right] \times S^{1}}$ defines a homotopically unique map $\Phi: S^{1} \times$ $[0,1] \rightarrow V$ satisfying $\left.\Phi\right|_{S^{1} \times 0}=\gamma^{-}$and $\left.\Phi\right|_{S^{1} \times 1}=\gamma^{+}$. We can assume that the homotopy class of $\Phi$ is independent of $n$. In the notation of Section 2.3 we distinguish the following two cases,

$$
\begin{array}{ll}
\text { C1 } & \Delta S_{\omega}\left(\gamma^{+}, \gamma^{-} ; \Phi\right)=0 \\
\text { C2 } & \Delta S_{\omega}\left(\gamma^{+}, \gamma^{-} ; \Phi\right)>0 .
\end{array}
$$

Case C1 We shall show in this case that $\gamma^{+}$and $\gamma^{-}$are geometrically the same, and that also their parameterizations are the same. Moreover, we shall show that in this case the limit map $f$ extends continuously to the circle $\Gamma$ which is associated to this node. Assume first that one of the asymptotic limits, say $\gamma^{-}$, is an $\mathbf{R}$-orbit. Let us show that $\gamma^{+}$is also an $\mathbf{R}$-orbit and $\gamma^{-}(t)=\gamma^{+}(t)$ for all $t$. Indeed, by assumption, $E_{\omega}\left(\left.\widehat{F}_{n}\right|_{\left[-\widehat{N}_{n}, \widehat{N}_{n}\right]}\right) \rightarrow 0$ and $E\left(\widehat{F}_{n}\right) \leq E_{0}$. Thus we may apply Proposition 5.7 and find for every $\sigma>0$ a constant $c>0$ so that $\widehat{f}_{n}(s, t) \in B_{\sigma}\left(\widehat{f}_{n}(0, t)\right)$ for all $(s, t) \in\left[-\widehat{N}_{n}+c, \widehat{N}_{n}-c\right] \times S^{1}$ and $n$ large enough. Since $\widehat{f}_{n}\left( \pm \widehat{N}_{n}, t\right) \underset{n \rightarrow \infty}{\rightarrow} \gamma^{ \pm}(t)$ we conclude that $\gamma^{+}(t)=\gamma^{-}(t)$ for all $t$. This also proves that the limit map $f$ continuously extends to the circle $\Gamma$ associated to this node. Similarly, this time using Lemma 5.14, one shows that if $\gamma^{-}$is a point, then also $\gamma^{+}$is a point and $\gamma^{-}=\gamma^{+}=p$. Moreover, for large $n$ the image $\widehat{F}_{n}\left(\left[-\widehat{N}_{n}, \widehat{N}_{n}\right] \times S^{1}\right)$ is contained in an arbitrary small neighborhood of a point $p^{*}=(a, p) \in \mathbb{R} \times V$.

Case C2 Assume now $\Delta S_{\omega}\left(\gamma^{+}, \gamma^{-} ; \Phi\right)=\delta>0$. We point out that the uniform energy bound implies a uniform upper bound for the periods of $\gamma^{ \pm}$. This implies, as we shall first show, an a priori lower bound for $\delta$. Namely, we have 
Lemma 10.9 There exists a "quantum constant" $\hbar=\hbar(E)>0$ such that

$$
\Delta S_{\omega}\left(\gamma^{+}, \gamma^{-} ; \Phi\right)>\hbar .^{5}
$$

Proof To prove the claim we argue indirectly and assume that we have sequences of orbits (or points) $\gamma_{k}^{ \pm}$with bounded periods and holomorphic cylinders $\widehat{F}_{n, k}:\left[-\widehat{N}_{n, k}, \widehat{N}_{n, k}\right] \times S^{1} \rightarrow \mathbb{R} \times V$ satisfying

$$
\begin{gathered}
\lim _{n \rightarrow \infty} \widehat{N}_{n, k}=\infty \\
\lim _{n \rightarrow \infty} \widehat{F}_{n, k}\left( \pm \widehat{N}_{n, k} \times S^{1}\right)=\gamma_{k}^{ \pm} \text {in } C^{\infty}\left(S^{1}\right) \\
\Delta S_{\omega}\left(\gamma_{k}^{+}, \gamma_{k}^{-} ; \Phi_{k}\right)=\delta_{k}>0
\end{gathered}
$$

and

$$
\delta_{k} \rightarrow 0 \quad \text { as } k \rightarrow \infty
$$

Here $\Phi_{k}$ is the relative homotopy class of $\widehat{f}_{n, k}$ which is well defined for large $n$. Now arguing as in $\mathrm{C} 1$ we can choose a diagonal subsequence

$$
\widehat{F}_{n, k_{n}}:\left[-\widehat{N}_{n, k_{n}}, \widehat{N}_{n, k_{n}}\right] \times S^{1} \rightarrow \mathbb{R} \times V
$$

which converges uniformly to a trivial cylinder over a periodic orbit $\gamma$, or to a point $\gamma \in \mathbb{R} \times V$, in the following sense. There exists a constant $c$ such that

$$
\sup _{\left[-\widehat{N}_{n, k_{n}}+c, \widehat{N}_{n, k_{n}}-c\right] \times S^{1}} d\left(\widehat{f}_{n, k_{n}}(s, t), \gamma(t)\right) \underset{\tau \rightarrow \infty}{\longrightarrow} 0
$$

if $\gamma$ is a periodic orbit, and

$$
\sup _{\left[-\widehat{N}_{n, k_{n}}+c, \widehat{N}_{n, k_{n}}-c\right] \times S^{1}} d\left(\widehat{F}_{n, k_{n}}(s, t),(0, \gamma)\right) \underset{\tau \rightarrow \infty}{\longrightarrow} 0
$$

if $\gamma(t)=\gamma$ is a point. In the latter case we assume the $\mathbb{R}$-component of the maps $\widehat{F}_{n, k_{n}}$ to be fixed by the condition $\widehat{F}_{n, k_{n}}(0,0)=0$. In the situation where $\gamma$ is a periodic orbit we recall that in view of compactness of $V$ and the MorseBott condition, the periods of $\gamma_{k_{n}}^{+}$and $\gamma_{k_{n}}^{-}$are equal to that of $\gamma$ for large $n$. Hence $\delta_{k_{n}}=\Delta S_{\omega}\left(\gamma_{k_{n}}^{+}, \gamma_{k_{n}}^{-} ; \Phi_{k_{n}}\right)=0$ for large $n$, in contradiction to the assumption. If $\gamma$ is a point we arrive at the same contradiction, hence proving the claim (36) above.

\footnotetext{
${ }^{5}$ Let us recall that we allow the orbits $\gamma^{ \pm}$be oriented by the vector field $-\mathbf{R}$ (see Remark 10.8). However, we always assume, that their orientation is chosen in such a way that $\Delta S_{\omega}\left(\gamma^{+}, \gamma^{-} ; \Phi\right)>0$.
} 
In view of Lemma 5.11 we may assume that the same $\hbar$ also serves as a lower bound of the $\omega$-energies of all holomorphic planes and spheres which appear as a result of the bubbling off analysis. Let us recall that the inequality (35) provides us with the uniform gradient bounds for the maps $\widehat{F}_{n}$. So, no bubbling off can occur anymore. Analogously to the broken trajectories in Morse theory and Floer theory we shall see that the worst which can happen in the limit is the splitting of our long cylinder into a finite sequence of cylinders, which, in the image under the map meet at their ends along periodic orbits $\gamma$ of the vector field $\mathbf{R}$. To see this we consider as in Case C1 a sequence $\widehat{F}_{n}:\left[-\widehat{N}_{n}, \widehat{N}_{n}\right] \times S^{1} \rightarrow$

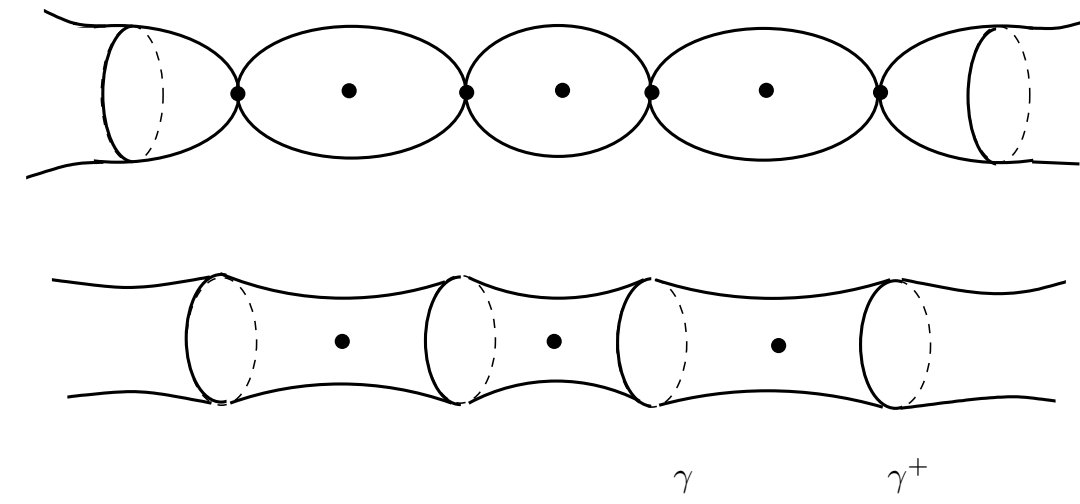

Figure 14: Splitting of a long cylinder

$\mathbb{R} \times V$ of cylinders having uniform gradient bounds and satisfying

$$
E_{\omega}\left(\widehat{F}_{n}\right)>\hbar,\left.\lim \widehat{f}_{n}\right|_{-\widehat{N}_{n} \times S^{1}}=\gamma^{-},\left.\lim \widehat{f}_{n}\right|_{\widehat{N}_{n} \times S^{1}}=\gamma^{+} .
$$

Then there exists $K_{n}<\widehat{N}_{n}$ such that

$$
\int_{\left[K_{n}, \widehat{N}_{n}\right]} \widehat{F}_{n}^{*} \omega=\hbar
$$

Then it follows from (34) that $\widehat{N}_{n}-K_{n} \rightarrow \infty$ as $n \rightarrow \infty$. Choose a point $p_{n}$ on the circle $K_{n} \times S^{1}$ and translate the $\mathbb{R}$-coordinate $\widehat{a}_{n}$ of the map $\widehat{F}_{n}=$ $\left(\widehat{a}_{n}, \widehat{f}_{n}\right)$ in such a way that $\widehat{a}_{n}\left(p_{n}\right)=0$. By means of Ascoli-Arzela theorem (a subsequence of) the sequence $\widehat{F}_{n}$ converges to a holomorphic cylinder $\widehat{F}_{\infty}: \mathbb{R} \times$ $S^{1} \rightarrow \mathbb{R} \times V$. If $\widehat{F}_{\infty}$ is a trivial cylinder over an orbit $\gamma$, or a constant map to a point, also denoted by $\gamma$, then $\Delta S_{\omega}\left(\gamma^{+}, \gamma ; \Phi\right)=\hbar$, where the homotopy class $\Phi$ is determined by the maps $\left.\widehat{F}_{n}\right|_{\left[K_{n}, \widehat{N}_{n}\right] \times S^{1}}$ for sufficiently large $n$. But this contradicts the a priori estimate (36). Hence the cylinder $\widehat{F}_{\infty}$ is not constant and different from the trivial cylinder over a periodic orbit, and hence has energy 
$E_{\omega}\left(\widehat{F}_{\infty}\right)>\hbar$. Consequently, by adding the extra marked points $\widehat{g}\left(p_{n}\right) \in S_{n}$ we create an additional spherical component $C$ of the limit Riemann surface $\mathbf{S}$. The limit map $\left.F_{\infty}\right|_{C}$ uses up more than $\hbar$ of the $\omega$-energy. Hence, iterating this analysis of long cylinders we arrive at the situation where the case C2 cannot occur anymore.

Behavior near a puncture We should also analyze the behavior of $F$ on a component of the thin part, adjacent to a marked point (puncture). Fix a positive $\varepsilon<\varepsilon_{0}$. The corresponding component $T_{n}$ of the $\varepsilon$-thin part of $S_{n}$ admits in this case a holomorphic parametrization

$$
g_{n}: A=[0, \infty) \times S_{1} \rightarrow\left(T_{n}, j_{n}\right)
$$

such that for every $n$ there exists a periodic orbit (or a point) $\gamma_{n}$ satisfying, in the $C^{\infty}\left(S^{1}\right)$-sense,

$$
\left.\lim _{s \rightarrow \infty} \widehat{F}_{n}\right|_{( \pm s) \times S^{1}}=\gamma_{n}
$$

where $\widehat{F}_{n}=\left(\widehat{a}_{n}, \widehat{f}_{n}\right)=F_{n} \circ \varphi_{n} \circ g_{n}$. Moreover, due to the compactness of $V$ and of the space of periodic orbits of bounded period we can assume that there exists a periodic orbit or a point $\bar{\gamma}=\lim _{n \rightarrow \infty} \gamma_{n}$. We can also assume an analogue to the inequality (35), namely

$$
\sup _{x \in A}\left\|\nabla \widehat{F}_{n}(x)\right\| \leq C,
$$

so that (a subsequence of) the sequence $\widehat{F}_{n}: A \rightarrow \mathbb{R} \times V$, normalized by the condition $\widehat{a}_{n}(0,0)=0$ converges in the $C_{\text {loc }}^{\infty}$-topology to a holomorphic map $\widehat{F}: A \rightarrow \mathbb{R} \times V$ asymptotic to a closed orbit, or a point $\underline{\gamma}$. Choose sequences $\underline{N}_{n}, \bar{N}_{n} \underset{n \rightarrow \infty}{\rightarrow} \infty$ and $\underline{N}_{n}<\bar{N}_{n}$, satisfying

$$
\left.\lim _{n \rightarrow \infty} \widehat{f}_{n}\right|_{\underline{N}_{n} \times S^{1}}=\underline{\gamma},\left.\quad \lim _{n \rightarrow \infty} \widehat{f}_{n}\right|_{\bar{N}_{n} \times S^{1}}=\bar{\gamma} .
$$

Notice that, given two sequences $\underline{N}_{n}^{\prime}, \bar{N}_{n}^{\prime} \underset{n \rightarrow \infty}{\rightarrow} \infty$ and a constant $c>0$ satisfying $\underline{N}_{n} \leq \underline{N}_{n}^{\prime} \leq \underline{N}_{n}+c$ and $\bar{N}_{n}-c \leq \bar{N}_{n}^{\prime} \leq \bar{N}_{n}$, we conclude

$$
\left.\lim _{n \rightarrow \infty} \widehat{f}_{n}\right|_{\underline{N}_{n}^{\prime} \times S^{1}}=\underline{\gamma},\left.\quad \lim _{n \rightarrow \infty} \widehat{f}_{n}\right|_{\bar{N}_{n}^{\prime} \times S^{1}}=\bar{\gamma} .
$$

For large $n$, the cylinder $\left.\widehat{f}_{n}\right|_{\left[\underline{N}_{n}, \bar{N}_{n}\right] \times S^{1}}$ defines a homotopically unique map $\Phi: S^{1} \times[0,1] \rightarrow V$ satisfying $\left.\Phi\right|_{S^{1} \times 0}=\underline{\gamma}$ and $\left.\Phi\right|_{S^{1} \times 1}=\bar{\gamma}$. As in the above analysis of a node adjacent to two components we distinguish the following two cases:

$$
\mathbf{C 1}^{\prime} \quad \Delta S_{\omega}(\underline{\gamma}, \bar{\gamma} ; \Phi)=0
$$




\section{C2 $^{\prime} \quad \Delta S_{\omega}(\underline{\gamma}, \bar{\gamma} ; \Phi)>0$.}

Because these cases are essentially similar to $\mathrm{C} 1$ and $\mathrm{C} 2$ we will restrict ourselves to the situation in which both $\underline{\gamma}$ and $\bar{\gamma}$ are $\mathbf{R}$-orbits.

Case C1 ${ }^{\prime}$ Let us show that in this case $\gamma(t)=\bar{\gamma}(t), t \in S^{1}$. Indeed, applying Proposition 5.7 we find for every $\varepsilon>0$ a constant $c>0$ such that

$$
\widehat{f}_{n}(s, t) \in B_{\varepsilon}\left(\widehat{f}_{n}\left(N_{n}, t\right)\right),
$$

where $N_{n}=\frac{\underline{N}+\bar{N}_{n}}{2}$, for all $(s, t) \in\left[\underline{N}_{n}+c, \bar{N}_{n}-c\right] \times S^{1}$ and $n$ large enough. Clearly, (see (38) above) $\lim _{n \rightarrow \infty} \widehat{f}_{n}\left(\underline{N}_{n}+c, t\right)=\lim _{n \rightarrow \infty} \widehat{f}_{n}\left(\underline{N}_{n}, t\right)=\underline{\gamma}(t)$ and $\lim _{n \rightarrow \infty} \widehat{f}_{n}\left(\bar{N}_{n}-c, t\right)=\lim _{n \rightarrow \infty} \widehat{f}_{n}\left(\bar{N}_{n}, t\right)=\bar{\gamma}(t), t \in S^{1}$. On the other hand, (39) implies that $\lim _{n \rightarrow \infty} \widehat{f}_{n}\left(\underline{N}_{n}+c, t\right)=\lim _{n \rightarrow \infty} \widehat{f}_{n}\left(\bar{N}_{n}-c, t\right)$. Hence, $\underline{\gamma}(t)=\bar{\gamma}(t), t \in S^{1}$. In fact, a similar argument implies a stronger statement. Namely, the sequence $\widehat{F}_{n}: A \rightarrow \mathbb{R} \times V$ converges to the holomorphic map $\widehat{F}$ uniformly, ie, for any $\varepsilon>0$ there exists $N, K>0$ such that $\widehat{f}_{n}(s, t) \in B_{\varepsilon}(\widehat{f}(s, t))$ for all $t \in S^{1}$, $n \geq K$ and $s \geq N$.

Case C2' The analysis of this case is identical to the case C2.

Manifolds with cylindrical ends and splitting The proof is essentially the same as for cylindrical manifolds. The only difference arises when, all the images $\widehat{F}_{n}\left(\left[-\widehat{N}_{n}, \widehat{N}_{n}\right] \times S^{1}\right)$ for large $n$ intersect the non-cylindrical part of $W$. In the analysis of Cases $\mathrm{C} 1$ and $\mathrm{C} 1^{\prime}$, when we need to show that if $\gamma^{-}$is a point then $\gamma^{+}$is the same point, we may use the Monotonicity Lemma 5.2 instead of Lemma 5.14. Similarly, the Monotonicity Lemma implies Lemma 10.9 for the case when either $\gamma^{-}$or $\gamma^{+}$is a point in the non-cylindrical part. The rest of analysis is the same with the only difference, that the notion of convergence in the non-cylindrical part does not involve any freedom of translation.

\subsubsection{Step 4: Level structure}

Cylindrical case Let us introduce an ordering in the set of components of $S \backslash \bigcup \Gamma_{i}$. Given two components $C_{i}$ and $C_{j}$, we choose two points $x_{i} \in C_{i}$ and $x_{j} \in C_{j}$, and define $C_{i} \leq C_{j}$ if

$$
\lim \sup _{n \rightarrow \infty}\left[a_{n} \circ \varphi_{n}\left(x_{i}\right)-a_{n} \circ \varphi_{n}\left(x_{j}\right)\right]<\infty .
$$

If $C_{i} \leq C_{j}$ and $C_{j} \leq C_{i}$, then we write $C_{i} \sim C_{j}$. Clearly, this ordering is independent of the choice of the points $x_{i}$ and $x_{j}$. Now, we can label the 
components $C_{i}$ with their level number as follows. The set of components minimal with respect to the above ordering will constitute level 1 . Then, after removing these components, the set of minimal components will be of level 2, etc. Clearly, this labelling is constant across nodes that are mapped at finite distance. However, it may happen that the level number jumps by an integer $N>1$ across a node in the limit. In that case, we have to insert $N-1$ additional components between these two components, each of them a vertical cylinder over the orbit corresponding to the above node. We can do this by adding additional points so that the images under the maps have the appropriate behavior. Finally, we remove the marked points that we added in all the previous steps of the proof. If level $i$ becomes unstable because of this, we remove it and decrease by 1 the labelling of higher levels. Hence, we obtain a level structure that satisfies all the conditions for a stable holomorphic building of height $k$ in the cylindrical almost complex manifold $(\mathbb{R} \times V, J)$.

Manifolds with cylindrical ends In every component $C_{i}$ we pick a point $x_{i} \in C_{i}$. We assign to a component $C_{i}$ the level number 0 if $F_{n} \circ \varphi_{n}\left(x_{i}\right)$ is contained in a compact part of $W$ for all $n$. This property is independent of the choice of the point $x_{i}$. For any other component $C_{i}$ the sequence $F_{n} \circ \varphi_{n}\left(x_{i}\right)$ is contained in one of the end components of $W$ for $n$ sufficiently large, and, in particular, $F_{n} \circ \varphi_{n}\left(x_{i}\right)$ can be written as $\left(a_{n} \circ \varphi_{n}\left(x_{i}\right), f_{n} \circ \varphi_{n}\left(x_{i}\right)\right)$. Let us introduce an ordering on the set of components $C_{i}$ associated to an end component $E$. We write $C_{i} \leq C_{j}$ if $\limsup _{n \rightarrow \infty}\left[a_{n} \circ \varphi_{n}\left(x_{i}\right)-a_{n} \circ \varphi_{n}\left(x_{j}\right)\right]<\infty$. If $C_{i} \leq C_{j}$ and $C_{j} \leq C_{i}$, then we write $C_{i} \sim C_{j}$. Clearly, this ordering is independent of the choice of the points $x_{i}$ and $x_{j}$. Now, we can label the components $C_{i}$ with their level number as follows. If $E$ is a positive end then the set of minimal components for the above ordering will be of level 1 . Then, after removing these components, the set of minimal components will be of level 2, etc. If $E$ is a negative end then the set of maximal components for the above ordering will be of level -1 . Then, after removing these components, the set of maximal components will be of level -2 , etc. Clearly, this labelling is constant across nodes that are mapped at finite distance. However, it may happen that the level number jumps by an integer $N>1$ across a node at infinity. In that case, we have to insert $N-1$ additional components between these two components, each of them a vertical cylinder over the orbit corresponding to the above node. Finally, remove the marked points that we added in all the previous steps of the proof. If level $i$ becomes unstable because of this, we remove it and for a positive (resp. negative) end decrease (resp. increase) by 1 the labelling of higher (resp. lower) levels. Hence, we obtain a level structure that satisfies all the necessary conditions for a stable holomorphic building of 
height $k_{-}|1| k_{+}$in the almost complex manifold $W$ with cylindrical ends.

Splitting Again choosing in each component $C_{i}$ a point $x_{i} \in C_{i}$ we assign to a component $C_{i}$ the main layer if there exists $N_{i}>0$ such that for sufficiently large $n$ we have

$$
F_{n} \circ \varphi_{n}\left(x_{i}\right) \in \stackrel{\circ}{W} \cup\left([-n, n] \backslash\left(-N_{i}, N_{i}\right)\right) \times V \subset W^{n} .
$$

For all the other components $F_{n} \circ \varphi_{n}\left(x_{i}\right)$ is contained inside the cylindrical part $[-n, n] \times V \subset W^{n}$ for large $n$, and hence one can define the partial order, and after that the labelling of the levels exactly as it was done in the cylindrical case above. The proofs of the Theorems 10.4-10.6 are complete.

\section{Other compactness results}

\subsection{Cylindrical structure over a non-compact manifold}

It is clear that the compactness theorem for holomorphic curves in a cylindrical almost complex manifold $(\mathbb{R} \times V, J)$ fails if $V$ is non-compact and if one does not impose some extra conditions on the behavior of $J$. In this section we discuss one type of conditions under which the compactness theorem can still be proven. A cooriented hypersurface $\Sigma \subset V$ is called pseudo-convex if $\widetilde{\Sigma}=\mathbb{R} \times \Sigma$ is a (non-strictly) pseudo-convex hypersurface in the almost complex manifold $\mathbb{R} \times V$.

Theorem 11.1 Let $(\mathbb{R} \times V, J)$ be a cylindrical almost complex manifold as in Theorem 10.1, except that $V$ is either

- compact manifold with a pseudo-convex boundary, or

- can be exhausted by compact domains with pseudo-convex boundaries.

Then for every $E>0$, the space $\overline{\mathcal{M}}_{g, \mu}(V) \cup\{E(F) \leq E\}$ is compact.

The proof of Theorem 10.1 obviously remains valid here because the pseudoconvex surfaces serve as barriers through which holomorphic curves cannot escape.

Here are some situations for which the pseudo-convexity condition for $\partial V$ is satisfied. 
Examples 11.2 a) If $\operatorname{dim} V=3$ and if the vector field $\underset{\widetilde{R}}{\mathbf{R}}$ is tangent to the hypersurface $\Sigma$, then $\Sigma$ is pseudo-convex. In fact, $\widetilde{\Sigma}$ is Levi-flat in this case. It is foliated by holomorphic cylinders $\mathbb{R} \times \gamma$ over trajectories $\gamma$ of the vector field $\mathbf{R} \in T \Sigma$.

b) If $\lambda_{T \Sigma}=0$, then $\Sigma$ is pseudo-convex. Again, $\widetilde{\Sigma}$ is Levi-flat in this case. This situation cannot, of course, appear in the contact case.

c) If $V=\mathbb{R}^{2 n-1}$ and $\lambda=d z+\frac{1}{2} \sum_{1}^{n-1} x_{i} d y_{i}-y_{i} d x_{i}$ is the standard contact form, then every geometrically convex (with respect to the standard affine structure) hypersurface in $V$ is pseudo-convex.

\subsection{Degeneration to the Morse-Bott case}

The Compactness Theorem 10.1 is limited to cylindrical almost complex manifolds with fixed $\omega, J$ and $\lambda$, satisfying either the Morse or the Morse-Bott condition of Section 2.3. In this section, we will state a compactness theorem providing a transition from the Morse to the Morse-Bott case. We consider a symmetric cylindrical almost complex manifold $(\mathbb{R} \times V, J)$ satisfying the MorseBott condition adjusted to a closed form $\omega$ on $V$. As in Section 2.3, we denote by $N_{T}$ the submanifolds of $V$ foliated by the $T$-periodic trajectories of the vector field $\mathbf{R}$. Let us fix $T_{0}>0$. Below we construct a special perturbation of $\mathbf{R}$ so that all but a finite number of closed orbits on $N_{T}$ for the periods $T \leq T_{0}$ are destroyed while the remaining closed orbits become non-degenerate. Let us choose a smooth function $G: V \rightarrow \mathbb{R}$ having the following properties for all periods $T \leq T_{0}$ :

(1) along the submanifolds $N_{T}$, we have $d G(\mathbf{R})=0$ and $d G(v)=0$ for every vector $v$ normal to $N_{T}$ with respect to the natural Riemannian metric $g(A, B)=\lambda(A) \cdot \lambda(B)+\omega(A, J B)$, where $A, B \in T V$,

(2) the restriction $\left.G\right|_{N_{T}}$ satisfies the Morse-Bott condition and the critical submanifolds of $\left.G\right|_{N_{T}}$ consist of finitely many closed $\mathbf{R}$-orbits.

For $\varepsilon$ small, consider perturbations $\omega_{\varepsilon}, \mathbf{R}_{\varepsilon}, J_{\varepsilon}$ of $\omega, \mathbf{R}$ and $J$, which are determined by the following properties:

- $\omega_{\varepsilon}=\omega+\varepsilon d(G \lambda)$

- $\left.\mathbf{R}_{\varepsilon}\right\lrcorner \omega_{\varepsilon}=0$ and $\lambda\left(\mathbf{R}_{\varepsilon}\right)=1$

- $J_{\varepsilon}=J$ on $\xi$ and $J_{\varepsilon} \frac{\partial}{\partial t}=\mathbf{R}_{\varepsilon}$. 
Writing

$$
\mathbf{R}_{\varepsilon}=\mathbf{R}+\varepsilon X_{\varepsilon}
$$

the vector field $X_{\varepsilon}$ satisfies $\lambda\left(X_{\varepsilon}\right)=0$ so that $X_{\varepsilon} \in \xi$. Moreover, we have

$$
\begin{aligned}
\left.X_{\varepsilon}\right\lrcorner \omega_{\varepsilon} & \left.\left.=\frac{1}{\varepsilon}\left(\mathbf{R}_{\varepsilon}\right\lrcorner \omega_{\varepsilon}-\mathbf{R}\right\lrcorner \omega_{\varepsilon}\right) \\
& \left.=-\frac{1}{\varepsilon}(\mathbf{R}\lrcorner(\omega+\varepsilon d G \wedge \lambda-G \varepsilon d \lambda)\right)=d G .
\end{aligned}
$$

In particular, the trajectories of $\mathbf{R}$ which belong to the critical point locus of $\left.G\right|_{N_{T}}$, remain periodic trajectories of $\mathbf{R}_{\varepsilon}$, while all other closed trajectories of period $\leq T_{0}$ got destroyed, as the next lemma states.

Lemma 11.3 For every $T_{0}>0$, there exists $\varepsilon_{0}>0$ so that, if $0<\varepsilon \leq \varepsilon_{0}$, the almost complex structure $J_{\varepsilon}$ satisfies the Morse condition for all closed $\mathbf{R}_{\varepsilon}-$ orbits of period less than $T_{0}$. Moreover, every closed $\mathbf{R}_{\varepsilon}$-orbit of period less than $T_{0}$ corresponds to a critical submanifold of $\left.G\right|_{N_{T}}$ for some $T<T_{0}$.

Note that in general $\left.\left(X_{\varepsilon}\right)\right\lrcorner d \lambda \neq 0$, so that the perturbed cylindrical almost complex manifold is not symmetric. However, this will not be an issue, because it is very close to be symmetric. Hence, the proof of Proposition 5.13 can be repeated almost verbatim. In particular, $J_{\varepsilon}$-holomorphic curves asymptotic to the closed $\mathbf{R}_{\varepsilon}$-orbits $\bar{\gamma}_{1}, \ldots, \bar{\gamma}_{k}$ and $\underline{\gamma}_{1}, \ldots, \underline{\gamma}_{l}$ corresponding to critical submanifolds of $\left.G\right|_{N_{T}}$ and representing a given homology class in $H_{2}\left(V, \bigcup \bar{\gamma}_{i} \cup \underline{\gamma}_{i}\right)$ have a uniformly bounded energy. On the other hand, observe that a $\bar{J}_{\varepsilon}{ }^{-}$ holomorphic curve $F$, that is asymptotic to at least one closed $\mathbf{R}_{\varepsilon}$-orbit which is not a critical submanifold of some $\left.G\right|_{N_{T}}$, satisfies

$$
E_{\lambda}(F)>T_{0} \text {. }
$$

In other words, the $J_{\varepsilon}$-holomorphic curves which satisfy the energy bound $E(F) \leq T_{0}$ are asymptotic only to the non-degenerate trajectories obtained by the above perturbation. Let $\gamma$ be a closed $\mathbf{R}$-orbit on $N_{T}$ and $\Delta s \in \mathbb{R}$. Observe that the gradient flow $\psi^{s}, s \in \mathbb{R}$, of the gradient vector field $\nabla G$ leaves invariant the submanifolds $N_{T}$ for $T \leq T_{0}$. Moreover, this flow leaves invariant also the foliation of $N_{T}$ into trajectories of $\mathbf{R}$. Let $\gamma=\gamma(t)$ be a periodic trajectory of $\mathbf{R}$ of period $T$ which is not critical for $G$. For $\Delta s>0$ we define the cylindrical gradient trajectory $\widetilde{G}_{\gamma, \Delta s}:[0, \Delta s] \times S^{1} \rightarrow V$ by the formula

$$
\widetilde{G}_{\gamma, \Delta s}(s, t)=\psi^{s}(\gamma(t)), s \in[0, \Delta s], t \in S^{1} .
$$

Thus the cylinder $\widetilde{G}_{\gamma, \Delta s}$ is swept by the gradient trajectories of the function $G$ starting at points of $\gamma$ and having length $\Delta s$. Next, let us define the objects 
that will be obtained as limits of $J_{\varepsilon}$-holomorphic maps, when $\varepsilon \rightarrow 0$. We start with the notion of a generalized holomorphic building of height 1, with $k^{\prime}$ sublevels. The notion is defined by the following four conditions (i)-(iv). Suppose we are given:

(i) $k^{\prime}$ nodal holomorphic curves

$$
\left(a_{i}, f_{i} ; S_{i}, M_{i}, D_{i}, \underline{Z}_{i} \cup \bar{Z}_{i}\right) \text {, where } i=1, \ldots, k^{\prime} ;
$$

(ii) $k^{\prime}+1$ collections of cylindrical gradient trajectories

$$
\left\{\widetilde{G}_{\gamma_{j, i}, \Delta s_{i}}, j=1, \ldots, p_{i}\right\} \text {, where } i=0, \ldots, k^{\prime},
$$

with $\Delta s_{0}=-\infty, \Delta s_{k^{\prime}}=+\infty$ and $0 \leq \Delta s_{i}<\infty$ for $i=1, \ldots k^{\prime}$. We denote by $\widetilde{S}_{i}$ their domains consisting of a collection of cylinders, and by $\widetilde{\Gamma}_{i}^{ \pm}$the sets of boundary circles corresponding to their positive and negative punctures;

(iii) the number $p_{i}^{+}$of positive punctures of $F_{i}$ and the number $p_{i+1}^{-}$of negative punctures of $F_{i+1}$ are equal to $p_{i}$ and for $i=1, \ldots, k^{\prime}$, there are orientation reversing diffeomorphisms $\Phi_{i}: \Gamma_{i}^{+} \rightarrow \widetilde{\Gamma}_{i}^{-}$and $\Psi_{i-1}: \widetilde{\Gamma}_{i-1}^{+} \rightarrow \Gamma_{i}^{-}$ which are orthogonal on each boundary component.

(iv) Gluing the $S_{i}^{Z_{i}}$ and $\widetilde{S}_{i}$ by means of the mappings $\Phi_{i}$ and the $\Psi_{i}$, we obtain a piecewise-smooth surface

$$
\bar{S}=\widetilde{S}_{0} \underset{\Psi_{0}}{\cup} S_{1}^{Z_{1}} \underset{\Phi_{1}}{\cup} \widetilde{S}_{1} \underset{\Psi_{1}}{\cup} \ldots \underset{\Phi_{k^{\prime}}}{\cup} \widetilde{S}_{k^{\prime}}
$$

The last condition in the definition of a generalized holomorphic building of height 1 in $\mathbb{R} \times V$, with $k^{\prime}$ sublevels i requires that the maps $\bar{f}_{i}$, for $i=1, \ldots, k^{\prime}$ and $\widetilde{G}_{\gamma_{j}, i, \Delta s_{i}}$, for $j=1, \ldots, p_{i}$ and $i=0, \ldots, k^{\prime}$, fit together into a continuous map $\bar{f}: \bar{S} \rightarrow V$.

Next we extend this definition to generalized holomorphic buildings of height $k$, with $k_{i}^{\prime}$ sublevels in level $i=1, \ldots, k$, by concatenating $k$ generalized holomorphic buildings $F_{i}$ of height 1 , with $k_{i}^{\prime}$ sublevels, as in Section 7.2. The stability condition for generalized holomorphic buildings means stability of all its sublevels in the sense of Section 7.1. We now extend the notion of convergence to a generalized holomorphic building. Suppose we are given a sequence $\left(F_{m}, \Phi_{m}\right)$, with $m \geq 1$, of $J_{\varepsilon_{m}}$-holomorphic buildings of height $\leq k$, where $\varepsilon_{m} \rightarrow 0$. The sequence $\left(F_{m}, \Phi_{m}\right)$ converges to a generalized $J$-holomorphic building $F$ of height $k$, with $k_{i}^{\prime}$ sublevels in level $i=1, \ldots, k$, if there exist sequences $M_{m}^{\prime}$ of extra sets of marked points for the curves $\left(F_{m}, \Phi_{m}\right)$ and a set $M^{\prime}$ of extra marked points for $(F, \Phi)$ which have the same cardinality $N$ 


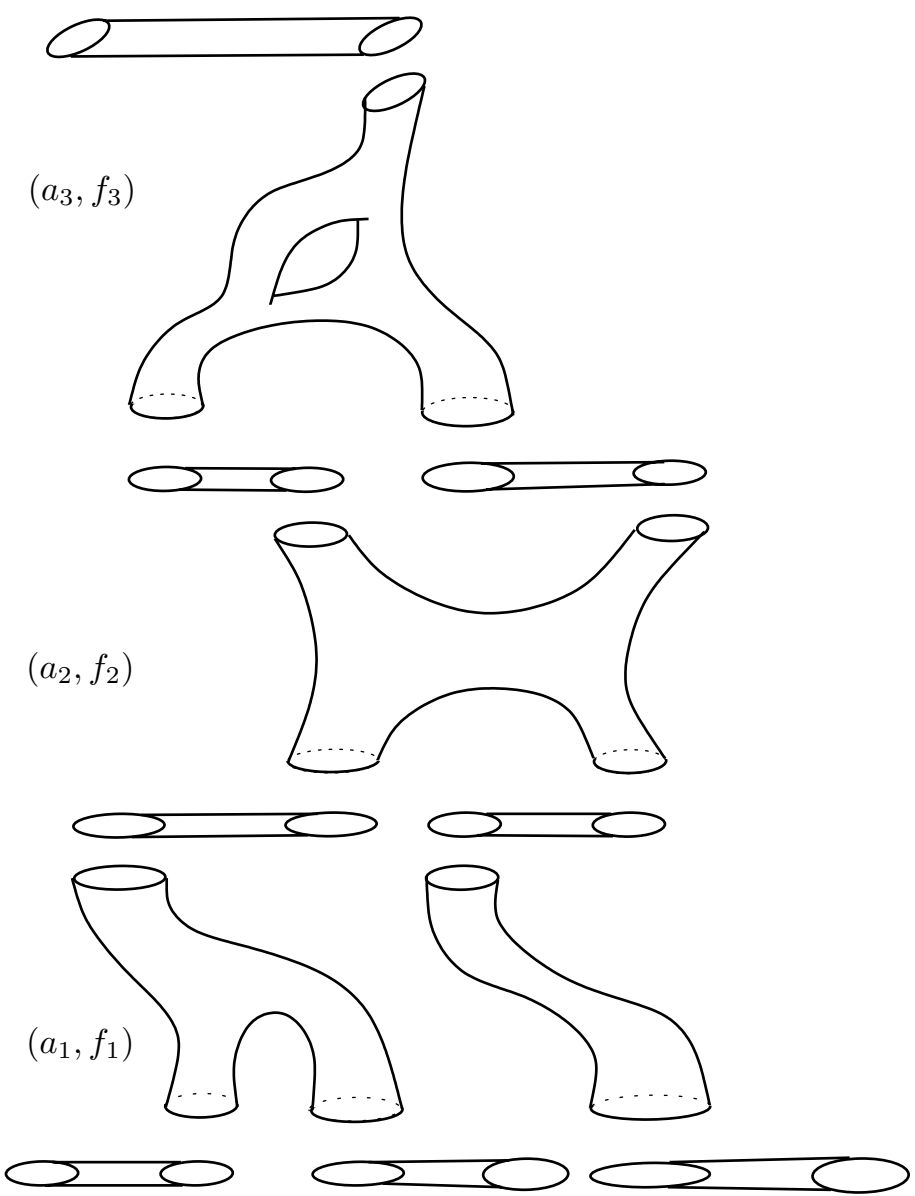

Figure 15: Generalized holomorphic building of height 1 with 3 sublevels

and which stabilize the corresponding underlying Riemann surfaces such that the following conditions are satisfied. Assume

$$
\left(\mathbf{S}_{F_{m}}, r_{F_{m}}, \Phi_{m}\right)=\left(S_{m}, j_{m}, M_{m} \cup M_{m}^{\prime}, D_{m}, r_{m}\right)
$$

and

$$
\left(\mathbf{S}_{F}, r_{F}, \Phi\right)=\left(S, j, M \cup M^{\prime}, D, r\right)
$$

are the decorated stable nodal Riemann surfaces which underly the curves diffeomorphisms $\varphi_{m}: S^{D, r} \rightarrow S^{D_{m}, r_{m}}$ with $\varphi_{m}(M)=M_{m}$ and $\varphi_{m}\left(M^{\prime}\right)=M_{m}^{\prime}$ which satisfy the conditions CRS1-CRS3 in the definition of the DeligneMumford convergence of Riemann surfaces and such that, in addition, the following conditions hold. 
CGHC1 For every component $C$ of $S^{D, r} \backslash \bigcup \Gamma_{i}$ which is not a cylinder corresponding to a gradient trajectory, the sequence of projections $f_{m} \circ$ $\left.\varphi_{m}\right|_{C}: C \rightarrow V$ converges to $\left.f\right|_{C}: C \rightarrow V$ in $C_{\mathrm{loc}}^{\infty}$.

CGHC2 If $C_{i, j}$ is the union of components of $S^{D, r} \backslash \bigcup \Gamma_{i}$ which correspond to the same sublevel $j=1, \ldots, k_{i}^{\prime}$ of the same level $i=1, \ldots, k$ of the building $F$, then there exist sequences $c_{m}^{i, j}$ for $i=1, \ldots, k$ and $j=$ $1, \ldots, k_{i}^{\prime}$, and $m \geq 1$, such that $\left.\left(a_{m} \circ \varphi_{m}-a-c_{m}^{i, j}\right)\right|_{C_{i, j}} \rightarrow 0$ in $C_{\mathrm{loc}}^{\infty}$.

With these definitions, the compactness theorem can be stated as follows.

Theorem 11.4 Assume $0<\varepsilon_{n} \leq \varepsilon_{0}$ and $\varepsilon_{n} \rightarrow 0$. Let

$$
\begin{gathered}
\mathbf{F}_{n}=\left(F_{n}=\left(a_{n}, f_{n}\right) ; S_{n}, j_{n}, M_{n}, \underline{Z}_{n} \cup \bar{Z}_{n}\right), \\
\bar{Z}_{n}=\left\{\left(\bar{z}_{1}\right)_{n}, \ldots,\left(\bar{z}_{p^{+}}\right)_{n}\right\}, \underline{Z}_{n}=\left\{\left(\underline{z}_{1}\right)_{n}, \ldots,\left(\underline{z}_{p^{-}}\right)_{n}\right\},
\end{gathered}
$$

be a sequence of smooth holomorphic curves in $\left(W=\mathbb{R} \times V, J_{\varepsilon_{n}}\right)$ of the same signature $\left(g, \mu, p^{-}, p^{+}\right)$and asymptotic at the corresponding punctures to the same orbits in $\mathcal{P}_{\varepsilon_{n}}$. Then there exists a subsequence that converges to a stable generalized holomorphic building $F$ of height $k$.

This theorem is proven in [1] and can be used to compute some symplectic and contact invariants in the Morse-Bott case.

\subsection{Compactness results in the Relative Symplectic Field The- ory}

In the relative Gromov-Witten theory one studies moduli spaces of holomorphic maps $((S, \partial S), j) \rightarrow((W, L), J)$ where $L$ is a totally real submanifold of the middle dimension. In case $J$ is tamed by a symplectic form $\omega$ one usually requires, in order to ensure compactness results, that $L$ is Lagrangian with respect to $\omega$, ie, $\left.\omega\right|_{L}=0$. In the context of the Symplectic Field Theory we consider a symmetric cylindrical manifold $(\mathbb{R} \times V, J)$ with $\operatorname{dim} V=2 n-1$ and with $J$ adjusted to a maximal rank closed 2-form $\omega$ on $V$. Suppose that $L$ is a $(n-1)$-dimensional isotropic submanifold for $\omega$, ie, $\left.\omega\right|_{L}=0$, and also integral for $\xi$, ie, $\left.\lambda\right|_{L}=0$. In the contact case, when $\omega=d \lambda$, the second condition implies the first one and just requires that $L$ is a Legendrian submanifold for the contact structure $\xi$. Set $\widehat{L}=\mathbb{R} \times L \subset \mathbb{R} \times V$. Given a Riemann surface $(S, j)$ with boundary $\partial S$ and with two sets of punctures $Z=\left\{z_{1}, \ldots, z_{s}\right\} \subset \operatorname{Int} S$ and $B=\left\{b_{1}, \ldots, b_{t}\right\} \subset \partial S$, we consider the moduli 
space of $(j, J)$-holomorphic maps $(S \backslash Z, \partial S \backslash B) \rightarrow(\mathbb{R} \times V, \widehat{L})$ such that near interior punctures from $Z$ the maps are asymptotic to periodic orbits of the Reeb vector field $\mathbf{R}$, and near the boundary punctures from $Z$, the maps are asymptotic to chords of $\mathbf{R}$ connecting two points on $L$, either at $+\infty$ or at $-\infty$. One can define the moduli spaces of holomorphic buildings of height $k$ and prove the corresponding compactness theorem, similar to Theorem 10.1, following the scheme of the absolute case. In fact, in the real analytic situation the relative compactness theorem can be formally deduced from the absolute one by using Gromov's doubling trick, see [8]. See also the paper [3] which is devoted to the Legendrian contact homology theory, and where the necessary compactness results are proven in a somewhat different setup. Suppose now $(W=\bar{W} \cup E, J)$ is an almost complex manifold with symmetric cylindrical end(s) where $J$ is adjusted to a symplectic form $\omega$ on $\bar{W}$. Let us consider a Lagrangian submanifold $\Lambda \subset \bar{W}$ which intersects the boundary $V=\partial \bar{W}$ transversally along a submanifold $L=\bar{W} \cup \Lambda$, integral for the distribution $\xi=\{\lambda=0\}$ on $V$. Then $\Lambda$ can be extended to the end $E$ as the cylindrical manifold $\widehat{L}=\mathbb{R} \times L$. We abbreviate $\widehat{\Lambda}=\Lambda \cup \widehat{L}$ and consider the moduli space of $(j, J)$-holomorphic maps $(S \backslash Z, \partial S \backslash B) \rightarrow(W, \widehat{\Lambda})$ such that near interior punctures from $Z$ the maps are asymptotic to periodic orbits of $\mathbf{R}$, and near the boundary punctures from $Z$ the maps are asymptotic to chords of $\mathbf{R}$ connecting two points on $L$, at one of the ends of $W$. The definition of the moduli spaces of holomorphic buildings of height $k_{-}|1| k_{+}$, the formulation and the proof of the relative analogue of Theorem 10.2 can be done following the same scheme, as in the absolute case.

\section{A Appendix : Asymptotic convergence estimates}

We shall first describe the structure of the manifold $V$ near a periodic orbit of Morse-Bott type.

Lemma A.1 Assuming the Morse-Bott situation, let $N$ denote a component of the set $N_{T} \subset V$ covered by periodic orbits of period $T$ of the vector field $\mathbf{R}$. Let $\gamma$ be one of the orbits from $N$. Then

a) if $T$ is the minimal period of $\gamma$ then there exists a neighborhood $U \supset \gamma$ in $V$ such that $U \cap N$ is invariant under the flow of $\mathbf{R}$ and one finds coordinates

$$
\left(\vartheta, x_{1}, \ldots, x_{n-1}, y_{1}, \ldots, y_{n-1}\right)
$$


such that

$$
\begin{gathered}
N=\left\{x_{1}, \ldots, x_{p}=0, y_{1}, \ldots, y_{q}=0\right\}, \text { for } 0 \leq p, q \leq n-1, \\
\left.\mathbf{R}\right|_{N}=\frac{\partial}{\partial \vartheta},
\end{gathered}
$$

and

$$
\left.\omega\right|_{N}=\left.\omega_{0}\right|_{N} \text { where } \omega_{0}=\sum_{1}^{n-1} d x_{i} \wedge d y_{i} .
$$

b) if $\gamma$ is a $m$-multiple of a trajectory $P$ of a minimal period $\frac{T}{m}$ there exists a tubular neighborhood $\widetilde{U}$ of $P$ such that its $m$-multiple cover $U$ together with all the structures induced by the covering map $\widetilde{U} \rightarrow U$ from the corresponding objects on $U$ satisfy the properties of the part a).

Proof In the case a) the orbit $\gamma$ has a neighborhood $U$ such that $U \cap N$ is fibrated by trajectories of $\mathbf{R}$. since $\mathbf{R}\lrcorner \omega=0$ and $d \omega=0$, the 2 -form $\left.\omega\right|_{N}$ descends to the quotient by $S^{1}$. By the Morse-Bott assumption the rank of of the form induced on this quotient is constant, and hence $N / S^{1}$ is foliated by isotropic submanifolds. The statement then follows using an appropriate version of Darboux theorem. In the situation of b) by taking the $m$-multiple cover of a neighborhood of $P$ we return to the situation considered in a).

Let $N$ be, as in the above lemma, a component consisting of periodic orbits of $\mathbf{R}$ of period $T$. Let $\gamma$ is one of the periodic orbits from $N$. Let $F=$ $(a, f):[-R,+R] \times S^{1} \rightarrow \mathbb{R} \times V$ be a $J$-holomorphic cylinder in a neighborhood of $\gamma$. If the minimal period of $\gamma$ is equal to $T / m$ then by taking the $m^{-}$ folded cover of a neighborhood of $\gamma$ and lifting there our holomorphic map we are in the situation in which $T$ is the minimal period of $\gamma$, and hence a neighborhood of $\gamma$ in $N$ is fibrated by the closed orbits of $\gamma$. We introduce in a neighborhood $U$ of $\gamma$ the local coordinates $\left(\vartheta, z_{\text {in }}, z_{\text {out }}\right) \in \mathbb{R} / \mathbb{Z} \times \mathbb{R}^{k} \times \mathbb{R}^{2 n-2-k}$, where $k=2 n-2-(p+q)$ and

$$
z_{\text {in }}=\left(x_{p+1}, \ldots, x_{n-1}, y_{q+1}, \ldots, y_{n-1}\right), \quad z_{\text {out }}=\left(x_{1}, \ldots, x_{p}, y_{1}, \ldots, y_{q}\right) .
$$

We will also set $z=\left(z_{\text {in }}, z_{\text {out }}\right)$ and $I_{0}=[-R,+R], \theta_{0}=I_{0} \times S^{1}$.

Lemma A.2 Near a closed $\mathbf{R}$-orbit, the Cauchy-Riemann equations can be written as follows,

$$
\begin{aligned}
z_{s}+M z_{t}+S z_{\text {out }} & =0 \\
a_{s}-T \vartheta_{t}+B z_{\text {out }}+B^{\prime} z_{t} & =0 \\
a_{t}+T \vartheta_{s}+C z_{\text {out }}+C^{\prime} z_{t} & =0 .
\end{aligned}
$$


Proof The Cauchy-Riemann equations are given by

$$
\frac{\partial F}{\partial s}+J \frac{\partial F}{\partial t}=0
$$

Let us first extract the $z$-components of this equation. The first term gives $z_{s}$, while the second term gives 3 contributions:

(1) $M z_{t}$, the $z$-components of $J z_{t}$, where $M=\left.\pi_{z} \circ J\right|_{z}$ and $\pi_{z}$ is the projection on the $z$ coordinates,

(2) $\pi_{z}\left(J \frac{\partial}{\partial \vartheta}\right) \vartheta_{t}$. Since $\frac{\partial}{\partial \vartheta}=T \mathbf{R}$ along $N_{T}$, this term has the form $S^{\prime} z_{\text {out }}$.

(3) $\pi_{z}\left(J \frac{\partial}{\partial t}\right) a_{t}$. Since $\pi_{z} \mathbf{R}=0$ along $N_{T}$, this term has the form $S^{\prime \prime} z_{\text {out }}$.

Combining all terms, we obtain the equation

$$
z_{s}+M z_{t}+S z_{\text {out }}=0
$$

where $S=S^{\prime}+S^{\prime \prime}$. Let us now extract the $a$ component of the CauchyRiemann equation. The first term gives $a_{s}$, while the second term gives 3 contributions:

(1) $\pi_{a} \circ J z_{t}=B^{\prime} z_{t}$, where $\pi_{a}$ is the projection on the $a$ coordinate.

(2) $\pi_{a}\left(J \frac{\partial}{\partial \vartheta}\right) \vartheta_{t}=-T \vartheta_{t}+B z_{\text {out }}$, since $J \frac{\partial}{\partial \vartheta}=-T \frac{\partial}{\partial a}$ along $N_{T}$.

(3) $\pi_{a}\left(J \frac{\partial}{\partial t}\right) a_{t}=0$.

Combining all terms, we obtain the equation

$$
a_{s}-T \vartheta_{t}+B z_{\text {out }}+B^{\prime} z_{t}=0 .
$$

Finally, we apply $J$ to the Cauchy-Riemann equation and extract the $a$ component from the resulting equation. The second term gives $-a_{t}$, while the first term gives 3 contributions:

(1) $\pi_{a}\left(J z_{s}\right)=C_{1} z_{\text {out }}+C^{\prime} z_{t}$ using the $z$-components of the Cauchy-Riemann equations.

(2) $\pi_{a}\left(J \frac{\partial}{\partial \vartheta}\right) \vartheta_{s}=-T \vartheta_{t}+C_{2} z_{\text {out }}$, since $J \frac{\partial}{\partial \vartheta}=-T \frac{\partial}{\partial a}$ along $N_{T}$.

(3) $\pi_{a}\left(J \frac{\partial}{\partial t}\right) a_{s}=0$.

Combining all terms and changing the sign, we obtain the equation

$$
a_{t}+T \vartheta_{t}+C z_{\text {out }}+C^{\prime} z_{t}=0
$$

where $C=C_{1}+C_{2}$. 
Define the linear operators

$$
A(s): H^{1}\left(S^{1}, \mathbb{R}^{2 n-2}\right) \subset L^{2}\left(S^{1}, \mathbb{R}^{2 n-2}\right) \rightarrow L^{2}\left(S^{1}, \mathbb{R}^{2 n-2}\right)
$$

by the formula

$$
(A(s) z)(t)=-M(u(s, t)) z_{t}(t)-S(u(s, t)) z_{\text {out }}(t),
$$

where we have abbreviated $u(s, t)=(a(s, t), f(s, t))$. Then equation (41) becomes $z_{s}(s, \cdot)=A(s) z(s, \cdot)$. Note that the explicit expression for this operator depends on the $J$-holomorphic map $F=(a, f)$ through the matrices $M$ and $S$. If we substitute instead $\vartheta(s, t)=\vartheta\left(s_{0}, 0\right)+t, a(s, t)=T s$, $z_{\text {out }}(s, t)=0$ and $z_{\text {in }}(s, t)=z_{\text {in }}\left(s_{0}, 0\right)$, for some $s_{0} \in I_{0}$, we obtain another operator $\widetilde{A}(s)$. We will denote the $\operatorname{limit}_{\lim _{s \rightarrow \infty}} \widetilde{A}(s)$ by $A_{0}$. We write $\left(A_{0} u\right)(t)=$ $-M_{0}(t) u_{t}(t)-S_{0}(t) u_{\text {out }}(t)$. This operator corresponds to the linearized CauchyRiemann equation along the closed $\mathbf{R}$-orbit $\gamma_{s_{0}}(t)=\left(\vartheta\left(s_{0}, 0\right)+t, z_{i n}\left(s_{0}, 0\right), 0\right)$. Revisiting the proof of Lemma A.2, we can see that $M_{0} S_{0} z_{\text {out }}$ is the linearization of the $z$-component of the vector field $\mathbf{R}$ along $\gamma_{s_{0}}$. Hence, the matrices $M_{0}(t), S_{0}(t)$ are in the symplectic algebra and the operator $A_{0}$ is self-adjoint with respect to the inner product

$$
\langle u, v\rangle_{0}=\int_{0}^{1}\left\langle u,-J_{0} M_{0} v\right\rangle d t
$$

where $J_{0}$ is the standard complex structure on $\mathbb{R}^{2 n-2}$ and $\langle\cdot, \cdot\rangle=\omega_{0}\left(\cdot, J_{0} \cdot\right)$. The kernel of $A_{0}$ is independent of $s_{0}$ and is generated by constant loops with values in the tangent space to $N$. Let $P_{0}$ be the orthonormal projection to ker $A_{0}$ with respect to $\langle\cdot, \cdot\rangle_{0}$, and $Q_{0}=I-P_{0}$. The operator $Q_{0}$ clearly has the following properties: $\left(Q_{0} z\right)_{t}=z_{t},\left(Q_{0} z\right)_{s}=Q_{0} z_{s},\left(Q_{0} z\right)_{\text {out }}=z_{\text {out }}$ and $Q_{0} A_{0}=A_{0} Q_{0}$. We will first obtain some estimates for the decaying rate of $z_{\text {out }}$. Abbreviate $g_{0}(s):=\frac{1}{2}\left\|Q_{0} z(s)\right\|_{0}^{2}$ and $\beta_{0}(s)=\left(\vartheta\left(s_{0}, 0\right)-\vartheta(s, 0), z_{i n}\left(s_{0}, 0\right)-z_{i n}(s, 0)\right)$.

Lemma A.3 There exist $\delta>0$ and $\bar{\beta}>0$ such that, if

$$
\sup _{(s, t) \in \theta_{0}}\left|\partial^{\alpha} z_{\text {out }}(s, t)\right| \leq \delta
$$

for multi-indices $\alpha$ with $|\alpha| \leq 2$, and

$$
\begin{aligned}
\sup _{(s, t) \in \theta_{0}}\left|\partial^{\alpha} z_{i n}(s, t)\right| & \leq \delta \\
\sup _{(s, t) \in \theta_{0}}\left|\partial^{\alpha}(\vartheta(s, t)-t)\right| & \leq \delta
\end{aligned}
$$

for those multi-indices $\alpha$ satisfying $0<|\alpha| \leq 2$, then for $s \in I_{0}$ satisfying $\left|\beta_{0}(s)\right| \leq \bar{\beta}$, we have

$$
g_{0}^{\prime \prime}(s) \geq c_{1}^{2} g_{0}(s),
$$

where $c_{1}>0$ is a constant independent of $s_{0}$. 
Proof Clearly,

$$
g_{0}^{\prime \prime}(s) \geq\left\langle Q_{0} z_{s s}, Q_{0} z\right\rangle_{0} .
$$

Let us compute the right hand side. First,

$$
\begin{aligned}
Q_{0} z_{s}= & Q_{0} A(s) z(s) \\
= & Q_{0} A_{0} z+Q_{0}\left[A(s)-A_{0}\right] z \\
= & Q_{0} A_{0} z+Q_{0}\left[\Delta_{0} z_{t}+\hat{\Delta}_{0} z_{\text {out }}+\left(\tilde{\Delta}_{0} \beta_{0}\right) z_{t}+\left(\bar{\Delta}_{0} \beta_{0}\right) z_{\text {out }}\right] \\
= & A_{0} Q_{0} z+Q_{0} \Delta_{0}\left(Q_{0} z\right)_{t}+Q_{0} \hat{\Delta}_{0}\left(Q_{0} z\right)_{\text {out }}+Q_{0}\left(\tilde{\Delta}_{0} \beta_{0}\right)\left(Q_{0} z\right)_{t} \\
& +Q_{0}\left(\bar{\Delta}_{0} \beta_{0}\right)\left(Q_{0} z\right)_{\text {out }},
\end{aligned}
$$

where

$$
\begin{aligned}
& \Delta_{0}=M\left(\vartheta(s, 0)+t, z_{i n}(s, 0), 0\right)-M(\vartheta(s, t), z(s, t)) \\
& \hat{\Delta}_{0}=S\left(\vartheta(s, 0)+t, z_{i n}(s, 0), 0\right)-S(\vartheta(s, t), z(s, t)),
\end{aligned}
$$

and where the matrices $\tilde{\Delta}_{0}(s, t), \bar{\Delta}_{0}(s, t)$ are defined via the mean value theorem applied to $M$ between points $\left(\vartheta\left(s_{0}, 0\right)+t, z_{i n}\left(s_{0}, 0\right)\right)$ and $\left(\vartheta(s, 0)+t, z_{i n}(s, 0)\right)$, and hence we have

$$
\begin{aligned}
& \tilde{\Delta}_{0} \beta_{0}=M_{0}-M\left(\vartheta(s, 0)+t, z_{i n}(s, 0), 0\right) \\
& \bar{\Delta}_{0} \beta_{0}=S_{0}-S\left(\vartheta(s, 0)+t, z_{i n}(s, 0), 0\right) .
\end{aligned}
$$

The expressions $\Delta_{0}$ and $\hat{\Delta}_{0}$ contain the dependence in $z_{\text {out }}$ so that

$$
\begin{aligned}
& \sup _{(s, t) \in \theta_{0}}\left|\partial^{\alpha} \Delta_{0}(s, t)\right| \leq C \delta \\
& \sup _{(s, t) \in \theta_{0}}\left|\partial^{\alpha} \hat{\Delta}_{0}(s, t)\right| \leq C \delta,
\end{aligned}
$$

for multi-indices $\alpha$ with $|\alpha| \leq 1$. On the other hand, the expressions $\tilde{\Delta}_{0}$ and $\bar{\Delta}_{0}$ contain the dependence in $z_{i n}$ and $\vartheta$. Therefore,

$$
\begin{aligned}
& \sup _{(s, t) \in \theta_{0}}\left|\partial^{\alpha} \tilde{\Delta}_{0}(s, t)\right| \leq C \\
& \sup _{(s, t) \in \theta_{0}}\left|\partial^{\alpha} \bar{\Delta}_{0}(s, t)\right| \leq C,
\end{aligned}
$$

Geometry 8 Topology, Volume 7 (2003) 
for multi-indices $\alpha$ with $|\alpha| \leq 1$. Taking the derivative once more, we obtain

$$
\begin{aligned}
Q_{0} z_{s s}= & A_{0}\left(Q_{0} z\right)_{s}+Q_{0}\left(\frac{\partial}{\partial s} \Delta_{0}\right)\left(Q_{0} z\right)_{t}+Q_{0} \Delta_{0}\left(Q_{0} z_{s}\right)_{t} \\
& +Q_{0}\left(\frac{\partial}{\partial s} \hat{\Delta}_{0}\right)\left(Q_{0} z\right)_{\text {out }}+Q_{0} \hat{\Delta}_{0}\left(\left(Q_{0} z\right)_{\text {out }}\right)_{s} \\
& +Q_{0}\left(\frac{\partial}{\partial s} \tilde{\Delta}_{0}\right) \beta_{0}\left(Q_{0} z\right)_{t}+Q_{0}\left(\tilde{\Delta}_{0} \frac{d}{d s} \beta_{0}\right)\left(Q_{0} z\right)_{t}+Q_{0}\left(\tilde{\Delta}_{0} \beta_{0}\right)\left(Q_{0} z_{s}\right)_{t} \\
& +Q_{0}\left(\frac{\partial}{\partial s} \bar{\Delta}_{0}\right) \beta_{0}\left(Q_{0} z\right)_{\text {out }}+Q_{0}\left(\bar{\Delta}_{0} \frac{d}{d s} \beta_{0}\right)\left(Q_{0} z\right)_{\text {out }} \\
& +Q_{0}\left(\bar{\Delta}_{0} \beta_{0}\right)\left(Q_{0} z_{s}\right)_{\text {out }} .
\end{aligned}
$$

Taking the inner product with $Q_{0} z$, we obtain

$$
\begin{aligned}
\left\langle Q_{0} z_{s s}, Q_{0} z\right\rangle_{0}= & \left\langle Q_{0} z_{s}, A_{0}\left(Q_{0} z\right)\right\rangle_{0}+\left\langle\left(\frac{\partial}{\partial s} \Delta_{0}\right)\left(Q_{0} z\right)_{t}, Q_{0} z\right\rangle_{0} \\
& +\left\langle\Delta_{0}\left(Q_{0} z_{s}\right)_{t}, Q_{0} z\right\rangle_{0} \\
& +\left\langle\left(\frac{\partial}{\partial s} \hat{\Delta}_{0}\right)\left(Q_{0} z\right)_{\text {out }}, Q_{0} z\right\rangle_{0}+\left\langle\hat{\Delta}_{0}\left(\left(Q_{0} z\right)_{\text {out }}\right)_{s}, Q_{0} z\right\rangle_{0} \\
& +\left\langle\left(\frac{\partial}{\partial s} \tilde{\Delta}_{0}\right) \beta_{0}\left(Q_{0} z\right)_{t}, Q_{0} z\right\rangle_{0}+\left\langle\left(\tilde{\Delta}_{0} \frac{d}{d s} \beta_{0}\right)\left(Q_{0} z\right)_{t}, Q_{0} z\right\rangle_{0} \\
& +\left\langle\left(\tilde{\Delta}_{0} \beta_{0}\right)\left(Q_{0} z_{s}\right)_{t}, Q_{0} z\right\rangle_{0} \\
& +\left\langle\left(\frac{\partial}{\partial s} \bar{\Delta}_{0}\right) \beta_{0}\left(Q_{0} z\right)_{\text {out }}, Q_{0} z\right\rangle_{0}+\left\langle\left(\bar{\Delta}_{0} \frac{d}{d s} \beta_{0}\right)\left(Q_{0} z\right)_{\text {out }}, Q_{0} z\right\rangle_{0} \\
& +\left\langle\left(\bar{\Delta}_{0} \beta_{0}\right)\left(Q_{0} z_{s}\right)_{\text {out }}, Q_{0} z\right\rangle_{0} .
\end{aligned}
$$

Let us denote the 11 terms of the right hand side by $T_{1}, \ldots, T_{11}$. Substituting $Q_{0} z_{s}$ by its value in $T_{1}$ we find

$$
\begin{aligned}
T_{1}= & \left\|A_{0} Q_{0} z\right\|_{0}^{2}+\left\langle Q_{0} \Delta_{0} Q_{0} z_{t}, A_{0} Q_{0} z\right\rangle_{0}+\left\langle Q_{0} \hat{\Delta}_{0}\left(Q_{0} z\right)_{\text {out }}, A_{0} Q_{0} z\right\rangle_{0} \\
& +\left\langle Q_{0}\left(\tilde{\Delta}_{0} \beta_{0}\right) Q_{0} z_{t}, A_{0} Q_{0} z\right\rangle_{0}+\left\langle Q_{0}\left(\bar{\Delta}_{0} \beta_{0}\right)\left(Q_{0} z\right)_{\text {out }}, A_{0} Q_{0} z\right\rangle_{0} .
\end{aligned}
$$

By integration by parts in $T_{3}$ and $T_{8}$,

$$
\begin{aligned}
T_{3} & =\left\langle\Delta_{0}\left(Q_{0} z_{s}\right)_{t}, Q_{0} z\right\rangle_{0} \\
& =\int_{0}^{1}\left\langle\left(Q_{0} z_{s}\right)_{t},-\Delta_{0}^{*} J_{0} M Q_{0} z\right\rangle d t \\
& =-\int_{0}^{1}\left\langle Q_{0} z_{s},\left(-\frac{\partial}{\partial t} \Delta_{0}^{*} J_{0} M\right) Q_{0} z\right\rangle d t-\int_{0}^{1}\left\langle Q_{0} z_{s},-\Delta_{0}^{*} J_{0} M Q_{0} z_{t}\right\rangle d t .
\end{aligned}
$$

Similarly,

$$
T_{8}=-\int_{0}^{1}\left\langle Q_{0} z_{s},\left(-\frac{\partial}{\partial t}\left(\tilde{\Delta}_{0} \beta_{0}\right) J_{0} M\right) Q_{0} z\right\rangle d t-\int_{0}^{1}\left\langle Q_{0} z_{s},-\left(\tilde{\Delta}_{0} \beta_{0}\right) J_{0} M Q_{0} z_{t}\right\rangle d t .
$$


Applying the Cauchy-Schwarz inequality to all terms $T_{i}$ and taking into account the bounds for $\Delta_{0}, \hat{\Delta}_{0}$ and for $\tilde{\Delta}_{0}, \bar{\Delta}_{0}$, we obtain

$$
\begin{aligned}
T_{1} \geq & \left\|A_{0} Q_{0} z\right\|_{0}^{2}-c \delta\left\|Q_{0} z_{t}\right\|_{0}\left\|A_{0} Q_{0} z\right\|_{0}-c \delta\left\|Q_{0} z\right\|_{0}\left\|A_{0} Q_{0} z\right\|_{0} \\
& -c\left|\beta_{0}\right|\left\|Q_{0} z_{t}\right\|_{0}\left\|A_{0} Q_{0} z\right\|_{0}-c\left|\beta_{0}\right|\left\|Q_{0} z\right\|_{0}\left\|A_{0} Q_{0} z\right\|_{0} . \\
T_{2} \geq & -c \delta\left\|Q_{0} z_{t}\right\|_{0}\left\|Q_{0} z\right\|_{0} . \\
T_{3} \geq & -c \delta\left\|Q_{0} z_{s}\right\|_{0}\left\|Q_{0} z\right\|_{0}-c \delta\left\|Q_{0} z_{s}\right\|_{0}\left\|Q_{0} z_{t}\right\|_{0} . \\
T_{4} \geq & -c \delta\left\|Q_{0} z\right\|_{0}^{2} . \\
T_{5} \geq & -c \delta\left\|Q_{0} z_{s}\right\|_{0}\left\|Q_{0} z\right\|_{0} . \\
T_{6} \geq & -c\left|\beta_{0}\right|\left\|Q_{0} z_{t}\right\|_{0}\left\|Q_{0} z\right\|_{0} . \\
T_{7} \geq & -c \delta\left\|Q_{0} z_{t}\right\|_{0}\left\|Q_{0} z\right\|_{0} . \\
T_{8} \geq & -c\left|\beta_{0}\right|\left\|Q_{0} z_{s}\right\|_{0}\left\|Q_{0} z\right\|_{0}-c\left|\beta_{0}\right|\left\|Q_{0} z_{s}\right\|_{0}\left\|Q_{0} z_{t}\right\|_{0} . \\
T_{9} \geq & -c\left|\beta_{0}\right|\left\|Q_{0} z\right\|_{0}^{2} . \\
T_{10} \geq & -c \delta\left\|Q_{0} z\right\|_{0}^{2} . \\
T_{11} \geq & -c\left|\beta_{0}\right|\left\|Q_{0} z_{s}\right\|_{0}\left\|Q_{0} z\right\|_{0} .
\end{aligned}
$$

Using the expression for $Q_{0} z_{s}$ we find

$$
\left\|Q_{0} z_{s}\right\|_{0} \leq\left\|A_{0} Q_{0} z\right\|_{0}+c \delta\left\|Q_{0} z_{t}\right\|_{0}+c \delta\left\|Q_{0} z\right\|_{0}+c\left|\beta_{0}\right|\left\|Q_{0} z_{t}\right\|_{0}+c\left|\beta_{0}\right|\left\|Q_{0} z\right\|_{0} .
$$

On the other hand, it is clear from the definition of $Q_{0}$ that

$$
\left\|A_{0} Q_{0} z\right\|_{0} \geq c_{1}\left(\left\|\left(Q_{0} z\right)_{t}\right\|_{0}^{2}+\left\|Q_{0} z\right\|_{0}^{2}\right)^{\frac{1}{2}} .
$$

Using the last 2 inequalities to eliminate $Q_{0} z, Q_{0} z_{s}$ and $Q_{0} z_{t}$ from the estimates for the $T_{i}$, we end up with

$$
\left\langle Q_{0} z_{s s}, Q_{0} z\right\rangle_{0} \geq\left(1-c \delta-c\left|\beta_{0}\right|\right)\left\|A_{0} Q_{0} z\right\|_{0}^{2} .
$$

Therefore, if $\delta$ and $\bar{\beta}$ are sufficiently small, then for $\left|\beta_{0}(s)\right|<\bar{\beta}$ we will have

$$
\left\langle Q_{0} z^{\prime \prime}(s), Q_{0} z(s)\right\rangle_{0} \geq \frac{1}{2}\left\|A_{0} Q_{0} z(s)\right\|_{0}^{2} .
$$

From this we deduce the desired estimate

$$
\begin{aligned}
g_{0}^{\prime \prime}(s) & \geq\left\langle Q_{0} z^{\prime \prime}(s), Q_{0} z(s)\right\rangle_{0} \\
& \geq \frac{1}{2}\left\|A_{0} Q_{0} z(s)\right\|_{0}^{2} \\
& \geq \frac{c_{1}^{2}}{2}\left\|Q_{0} z\right\|_{0}^{2}=c_{1}^{2} g_{0}(s) .
\end{aligned}
$$

Define $s_{1}=\sup \left\{s \in I_{0}|| \beta_{0}\left(s^{\prime}\right) \mid \leq \bar{\beta}\right.$ for all $\left.s^{\prime} \in\left[s_{0}, s\right]\right\}$. Then Lemma A.3 implies the following estimate. 
Corollary A.4

$$
g_{0}(s) \leq \max \left(g_{0}\left(s_{0}\right), g_{0}\left(s_{1}\right)\right) \frac{\cosh \left(c_{1}\left(s-\frac{s_{0}+s_{1}}{2}\right)\right)}{\cosh \left(c_{1} \frac{s_{0}-s_{1}}{2}\right)}
$$

for $s \in\left[s_{0}, s_{1}\right]$.

Proof Let us assume for determinacy that the maximum $\max \left(g_{0}\left(s_{0}\right), g_{0}\left(s_{1}\right)\right)$ is achieved at $s_{0}$, so that $g_{0}\left(s_{0}\right) \geq g_{0}\left(s_{1}\right)$. Let us denote the right hand side in (44) by $g_{1}(s)$. This function satisfies $g_{1}^{\prime \prime}(s)=c_{1}^{2} g_{1}(s), g_{1}\left(s_{0}\right)=g_{0}\left(s_{0}\right)$ and $g_{1}\left(s_{1}\right) \geq g_{0}\left(s_{1}\right)$. Therefore, the difference $g(s)=g_{0}(s)-g_{1}(s)$ satisfies $g^{\prime \prime}(s) \geq c_{1}^{2} g(s)$ for $s \in\left[s_{0}, s_{1}\right]$, vanishes at $s_{0}$ and is non-positive at $s_{1}$. The differential inequality implies that $g(s)$ cannot have a positive local maximum for $s_{0}<s<s_{1}$. Indeed, if $g$ is positive on the open sub-interval $\Delta \subset\left(s_{0}, s_{1}\right)$ and vanishes on its boundary, then there is point $a \in \Delta$ at which $g^{\prime \prime}(a)<0$, while $g(a)>0$, which contradicts the differential inequality. Hence, $g$ is nonpositive on $\left[s_{0}, s_{1}\right]$.

Next we derive some estimates for $z_{i n}$. Let $e$ be a unit vector in $\mathbb{R}^{2 n-2}$ with $e_{\text {out }}=0$.

Lemma A.5 Under the assumptions of Lemma A.3 and for $s \in\left[s_{0}, s_{1}\right]$, we have

$$
\left|\langle z(s), e\rangle_{0}-\left\langle z\left(s_{0}\right), e\right\rangle_{0}\right| \leq \frac{4 d}{c_{1}} \max \left(\left\|Q_{0} z\left(s_{0}\right)\right\|_{0},\left\|Q_{0} z\left(s_{1}\right)\right\|_{0}\right) .
$$

Proof The inner product of the Cauchy-Riemann equation (41) with $e$ gives

$$
\frac{d}{d s}\langle z, e\rangle_{0}+\left\langle M z_{t}, e\right\rangle_{0}+\left\langle S z_{\text {out }}, e\right\rangle_{0}=0 .
$$

But we have

$$
\begin{aligned}
\left\langle M z_{t}, e\right\rangle_{0} & =\int_{0}^{1}\left\langle M\left(Q_{0} z\right)_{t},-J_{0} M_{0} e\right\rangle d t \\
& =\int_{0}^{1}\left\langle Q_{0} z, \frac{d}{d t}\left(M^{*} J_{0} M_{0}\right) e\right\rangle d t
\end{aligned}
$$

so that

$$
\left|\left\langle M z_{t}, e\right\rangle_{0}\right| \leq d_{1}\left\|Q_{0} z\right\|_{0}
$$

Similarly,

$$
\left\langle S\left(Q_{0} z\right)_{\text {out }}, e\right\rangle_{0}=\int_{0}^{1}\left\langle\left(Q_{0} z\right)_{\text {out }}, S^{*}\left(-J_{0}\right) M_{0} e\right\rangle d t
$$


so that

$$
\left|\left\langle S z_{\text {out }}, e\right\rangle_{0}\right| \leq d_{2}\left\|Q_{0} z\right\|_{0}
$$

Therefore,

$$
\langle z(s), e\rangle_{0}-\left\langle z\left(s_{0}\right), e\right\rangle_{0} \leq d \int_{s_{0}}^{s}\left\|Q_{0} z(\sigma)\right\|_{0} d \sigma
$$

for $s \in I_{0}$. By equation (44),

$$
\left\|Q_{0} z(\sigma)\right\|_{0} \leq \max \left(\left\|Q_{0} z\left(s_{0}\right)\right\|_{0},\left\|Q_{0} z\left(s_{1}\right)\right\|_{0}\right) \sqrt{\frac{\cosh \left(c_{1}\left(\sigma-\frac{s_{0}+s_{1}}{2}\right)\right)}{\cosh \left(c_{1} \frac{s_{0}-s_{1}}{2}\right)}}
$$

for $\sigma \in\left[s_{0}, s_{1}\right]$. Hence, substituting in the integral and using the fact that $\sqrt{\cosh u}<\sqrt{2} \cosh \frac{u}{2}$, we obtain

$$
\left|\langle z(s), e\rangle_{0}-\left\langle z\left(s_{0}\right), e\right\rangle_{0}\right| \leq \frac{4 d}{c_{1}} \max \left(\left\|Q_{0} z\left(s_{0}\right)\right\|_{0},\left\|Q_{0} z\left(s_{1}\right)\right\|_{0}\right),
$$

since

$$
\sqrt{2} \frac{\sinh \left(c_{1} \frac{s_{0}-s_{1}}{4}\right)}{\sqrt{\cosh \left(c_{1} \frac{s_{0}-s_{1}}{2}\right)}} \leq 1
$$

Next, we shall derive estimates for the derivatives of $z$.

Lemma A.6 There exist $\delta>0$ and $\bar{\beta}>0$ such that, if

$$
\sup _{(s, t) \in \theta_{0}}\left|\partial^{\alpha} z_{\text {out }}(s, t)\right| \leq \delta
$$

for multi-indices $\alpha$ with $|\alpha| \leq k+2$ and

$$
\begin{aligned}
\sup _{(s, t) \in \theta_{0}}\left|\partial^{\alpha} z_{i n}(s, t)\right| & \leq \delta \\
\sup _{(s, t) \in \theta_{0}}\left|\partial^{\alpha}(\vartheta(s, t)-t)\right| & \leq \delta
\end{aligned}
$$

for multi-indices $\alpha$ with $0<|\alpha| \leq k+2$, then

$$
\begin{aligned}
& \left\|\partial^{\alpha} z(s)\right\|_{0} \\
& \quad \leq C_{\alpha} \max _{\left|\alpha^{\prime}\right| \leq|\alpha|}\left(\left\|Q_{0} \partial^{\alpha^{\prime}} z\left(s_{0}\right)\right\|_{0},\left\|Q_{0} \partial^{\alpha^{\prime}} z\left(s_{1}\right)\right\|_{0}\right) \sqrt{\frac{\cosh \left(c_{1}\left(s-\frac{s_{0}+s_{1}}{2}\right)\right)}{\cosh \left(c_{1} \frac{s_{0}-s_{1}}{2}\right)}},
\end{aligned}
$$

for all $s \in\left[s_{0}, s_{1}\right]$ and for every multi-index $\alpha$ with $1 \leq|\alpha| \leq k$. 
Proof The Cauchy-Riemann equation (41) can be written as

$$
\begin{aligned}
z_{s} & =A(s) z \\
& =A_{\infty} z+\bar{\Delta} z_{t}+\Delta z_{\text {out }},
\end{aligned}
$$

where $\Delta=S_{0}-S$ and $\bar{\Delta}=M_{0}-M$. Applying the projection $Q_{0}$ to this equation, we obtain for $w=\mathbb{Q}_{0} z$,

$$
w_{s}=A_{0} w+Q_{0} \bar{\Delta} w_{t}+Q_{0} \Delta w_{\text {out }} .
$$

where $w=Q_{0} z$. Let $W$ be the vector obtained by catenating $\left(\frac{\partial}{\partial s}\right)^{i}\left(A_{0}\right)^{j} w$ for $0 \leq i, j \leq k$. Then $W$ satisfies an equation of the same type,

$$
W_{s}=\mathcal{A}_{0} W+\mathcal{Q}_{0} \tilde{\Delta} W_{t}+\mathcal{Q}_{0} \hat{\Delta} W_{\text {out }},
$$

where $\mathcal{A}_{0}=\operatorname{diag}\left(A_{0}, \ldots, A_{0}\right)$ and $\mathcal{Q}_{0}=\operatorname{diag}\left(Q_{0}, \ldots, Q_{0}\right)$. Therefore, using the same estimates as in Lemma A.3, we obtain

$$
\|W(s)\|_{0} \leq C \max \left(\left\|W\left(s_{0}\right)\right\|_{0},\left\|W\left(s_{1}\right)\right\|_{0}\right) \sqrt{\frac{\cosh \left(c_{1}\left(s-\frac{s_{0}+s_{1}}{2}\right)\right)}{\cosh \left(c_{1} \frac{s_{0}-s_{1}}{2}\right)}} .
$$

Next we estimate $P_{0} z$ and its derivatives. Applying $P_{0}$ to the Cauchy-Riemann equation (41), we get

$$
\left(P_{0} z\right)_{s}=P_{0} \bar{\Delta}\left(Q_{0} z\right)_{t}+P_{0} \Delta\left(Q_{0} z\right)_{\text {out }} .
$$

We can apply $\left(\frac{\partial}{\partial s}\right)^{i}$, for $i=0, \ldots, k-1$ to this equation, and express the derivatives of $P_{0} z$ in terms of components of $W$, to obtain the desired estimate.

We now derive estimates for $\vartheta$.

Lemma A.7 Under the assumptions of Lemma A.6 with $k=1$,

$$
\int_{0}^{1}\left|\vartheta(s, \tau)-\vartheta\left(s_{0}, \tau\right)\right| d \tau \leq C \max _{|\alpha| \leq 1}\left(\left\|Q_{0} \partial^{\alpha} z\left(s_{0}\right)\right\|_{0},\left\|Q_{0} \partial^{\alpha} z\left(s_{1}\right)\right\|_{0}\right)
$$

for all $s \in\left[s_{0}, s_{1}\right]$.

Proof Consider the Cauchy-Riemann equations (42) and (43) for $a$ and $\vartheta$ :

$$
\left\{\begin{array}{l}
a_{s}-T \vartheta_{t}=-B\left(Q_{0} z\right)_{\text {out }}-B^{\prime}\left(Q_{0} z\right)_{t} \\
a_{t}+T \vartheta_{s}=-C\left(Q_{0} z\right)_{\text {out }}-C^{\prime}\left(Q_{0} z\right)_{t} .
\end{array}\right.
$$

If $s \in\left[s_{0}, s_{1}\right]$, the right hand side is bounded in norm as in equation (45). Therefore, integrating the second equation over $t$, we obtain

$$
\int_{0}^{1} \vartheta_{s} d t \leq C \max _{|\alpha| \leq 1}\left(\left\|Q_{0} \partial^{\alpha} z\left(s_{0}\right)\right\|_{0},\left\|Q_{0} \partial^{\alpha} z\left(s_{1}\right)\right\|_{0}\right) \sqrt{\frac{\cosh \left(c_{1}\left(s-\frac{s_{0}+s_{1}}{2}\right)\right)}{\cosh \left(c_{1} \frac{s_{0}-s_{1}}{2}\right)}} .
$$


Integrating over $s$, as in Lemma A.5, we find

$$
\int_{0}^{1}\left|\vartheta(s, \tau)-\vartheta\left(s_{0}, \tau\right)\right| d \tau \leq C^{\prime} \max _{|\alpha| \leq 1}\left(\left\|Q_{0} \partial^{\alpha} z\left(s_{0}\right)\right\|_{0},\left\|Q_{0} \partial^{\alpha} z\left(s_{1}\right)\right\|_{0}\right) .
$$

We are now in position to prove Proposition 5.7.

Proof of Proposition 5.7 Let $\hbar$ is chosen as in Lemma 5.11. Suppose also that the neighborhood $U$ is chosen so small that it satisfies the condition of Lemma A.1 and besides that it contains no periodic orbits of $\mathbf{R}$ of period $<2 T$, other than those which form the component $N=N_{T} \ni \gamma$. By contradiction, assume that there exists a sequence $F_{n}=\left(a_{n}, f_{n}\right):[-n,+n] \times S^{1} \rightarrow \mathbb{R} \times V$ of holomorphic cylinders, satisfying $E\left(F_{n}\right) \leq E_{0}$ and $E_{\omega}\left(F_{n}\right) \leq \hbar$, and a sequence $c_{n} \rightarrow \infty, c_{n}<n$ such that $f_{n}\left(s_{n}, t\right) \notin B_{\varepsilon}\left(f_{n}(0, t)\right)$ for some $s_{n} \in\left[-k_{n}, k_{n}\right]$, $k_{n}=n-c_{n}$. By Lemma 5.11, the gradient of $F_{n}$ is uniformly bounded on each compact subset, otherwise we would obtain a bubble with the $\omega$-energy exceeding $\hbar$. Hence, by Ascoli-Arzela, we can extract a subsequence converging to a holomorphic cylinder $F=: \mathbb{R} \times S^{1} \rightarrow \mathbb{R} \times V$, which is necessarily a trivial vertical cylinder over an orbit $\hat{\gamma} \in N$. Indeed, both asymptotic limits $\hat{\gamma}^{ \pm}$of this cylinder have to belong to $N_{T}$. This forces the equality $E_{\omega}(F)=0$ which then implies that $F$ is a trivial vertical cylinder. Let us show that

$$
\sup _{(s, t) \in\left[-k_{n}, k_{n}\right] \times S^{1}}\left|\partial^{\alpha} z_{\text {out }, n}(s, t)\right| \rightarrow 0
$$

for multi-indices $\alpha$ with $|\alpha| \geq 0$ and

$$
\begin{aligned}
\sup _{(s, t) \in\left[-k_{n}, k_{n}\right] \times S^{1}}\left|\partial^{\alpha} z_{i n, n}(s, t)\right| & \rightarrow 0 \\
\sup _{(s, t) \in\left[-k_{n}, k_{n}\right] \times S^{1}}\left|\partial^{\alpha}\left(\vartheta_{n}(s, t)-t\right)\right| & \rightarrow 0
\end{aligned}
$$

for multi-indices $\alpha$ with $|\alpha| \geq 1$, when $n \rightarrow \infty$. If this were not the case, we could translate the coordinates to center them on a sequence of points violating one of these properties. The sequence of cylinders obtained in this way would converge, as shown above, but the limit could not be a vertical cylinder, giving a contradiction. Hence, for $n$ sufficiently large, the suprema in equations (46), (47) and (48) for $|\alpha| \leq 3$ (and $|\alpha| \geq 1$ in cases (47) and (48)) will be smaller than a given $\delta>0$. Taking $R=k_{n}$, we can then apply the Lemmas A.3, A.5 and A.6 with $k=1$ to the cylinders $F_{n}:\left[-k_{n},+k_{n}\right] \times S^{1} \rightarrow \mathbb{R} \times V$. Since equations (46) and (47) imply that $\left\|Q_{0} z_{n}(s)\right\| \rightarrow 0$ as $n \rightarrow \infty$, Lemma A.6 gives uniform convergence of $\left\|\partial^{\alpha} z_{n}(s)\right\|_{0}$ to 0 , for $s$ satisfying $\left|\beta_{0}(s)\right| \leq \bar{\beta}$. By the Sobolev embedding theorem, these norm bounds imply pointwise bounds 
for $|z(s, t)-z(0, t)|$. Moreover, Lemma A.7 shows that $\left|\vartheta_{n}(s, t)-\vartheta_{n}(0, t)\right| \rightarrow 0$ uniformly where $\beta_{0}(s) \leq \bar{\beta}$, when $n \rightarrow \infty$. Hence, for $n$ sufficiently large, the property $\beta_{0}(s) \leq \bar{\beta}$ will be satisfied for all $s \in\left[-k_{n}, k_{n}\right]$, so the pointwise estimates hold for the whole $\left[-k_{n}, k_{n}\right] \times S^{1}$. But this contradicts the initial assumption that $f_{n}\left(s_{n}, t\right) \notin B_{\varepsilon}\left(f_{n}(0, t)\right)$ for some $s_{n} \in\left[-k_{n}, k_{n}\right]$.

\section{B Appendix: Metric structures on the compactified moduli spaces}

\section{B.1 Metrics on $\overline{\mathcal{M}}_{g, \mu}^{\$}$ and $\overline{\mathcal{M}}_{g, \mu}$}

The space $\overline{\mathcal{M}}_{g, \mu}$ is the standard Deligne-Mumford compactification of the moduli space $\mathcal{M}_{g, \mu}$. As it was shown by Wolpert (see [28]), the completion of $\mathcal{M}_{g, \mu}$ in the Weil-Petersson metric on $\mathcal{M}_{g, \mu}$ coincides with $\overline{\mathcal{M}}_{g, \mu}$, and thus the completed metric can be used to metrize the space $\overline{\mathcal{M}}_{g, \mu}$. As a topological space, $\overline{\mathcal{M}}_{g, \mu}^{\$}$ can be defined as an oriented blow-up of $\overline{\mathcal{M}}_{g, \mu}$ along the divisor corresponding to nodal surfaces. Hence it also can be metrized, though not in a canonical way. In this Appendix we define different metrics on $\overline{\mathcal{M}}_{g, \mu}$ and $\overline{\mathcal{M}}_{g, \mu}^{\$}$ compatible however with the same topologies on these moduli spaces. These metrics are more suitable for our further considerations of moduli spaces of holomorphic curves. Choose an $\varepsilon \leq \frac{\varepsilon_{0}}{2}$. Given (the equivalency classes of) two decorated nodal surfaces

$$
(\mathbf{S}, r)=(S, j, M, D, r) \quad \text { and } \quad\left(\mathbf{S}^{\prime}, r^{\prime}\right)=\left(S^{\prime}, j^{\prime}, M^{\prime}, D^{\prime}, r^{\prime}\right)
$$

we take their deformations $S^{D, r}$ and $\left(S^{\prime}\right)^{D^{\prime}, r^{\prime}}$, and consider the $\varepsilon$-thick parts $\operatorname{Thick}_{\varepsilon}(\mathbf{S})$ and $\operatorname{Thick}_{\varepsilon}\left(\mathbf{S}^{\prime}\right)$ of $\mathbf{S}$ and $\mathbf{S}^{\prime}$, viewed as subsets of $\dot{S}^{D, r}$ and $\left(\dot{S}^{\prime}\right)^{D^{\prime}, r^{\prime}}$. Thus each compact, ie, not adjacent to the punctures, component, $C_{i}$ of the thin part of $\dot{S}^{D, r}$, for $i=1, \ldots, N_{\varepsilon}$ (resp. $C_{i}^{\prime}$ of the thin part of $\left(\dot{S}^{\prime}\right)^{D^{\prime}, r^{\prime}}$, for $\left.i=1, \ldots, N_{\varepsilon}^{\prime}\right)$, contains the circle $\Gamma_{i}=\Gamma_{C_{i}}\left(\right.$ resp. $\left.\Gamma_{i}^{\prime}=\Gamma_{C_{i}^{\prime}}\right)$ which is either a closed geodesic or one of the special circles corresponding to the double points from $D$ (resp. $D^{\prime}$ ). Let us denote by $\mathcal{H}_{\varepsilon}\left(\mathbf{S}, \mathbf{S}^{\prime}\right)$ the (possibly empty) set of homeomorphisms $\dot{S}^{D, r} \rightarrow\left(\dot{S}^{\prime}\right)^{D^{\prime}, r^{\prime}}$ which map $\operatorname{Thick}_{\varepsilon}(\mathbf{S})$ quasi-conformally onto $\operatorname{Thick}_{\varepsilon}\left(\mathbf{S}^{\prime}\right)$. The homeomorphism $\varphi$ must preserve the ordering of cusps of $\mathbf{S}$ and $\mathbf{S}^{\prime}$ which correspond to the sets of marked points $M$ and $M^{\prime}$, while the ordering of circles $\Gamma_{i}$ and $\Gamma_{i}^{\prime}$ is irrelevant. For $\varphi \in \mathcal{H}_{\varepsilon}\left(\mathbf{S}, \mathbf{S}^{\prime}\right)$ we denote by $K_{\varepsilon}(\varphi)$ the maximal conformal distortion of $\varphi$ restricted to $\operatorname{Thick}_{\varepsilon}(\mathbf{S})$. If $\varphi$ is smooth,

$$
K_{\varepsilon}(\varphi)=\max _{x \in \operatorname{Thick}_{\varepsilon}(\mathbf{S})}\left|\log \lambda_{1}(x)-\log \lambda_{2}(x)\right|
$$


where $\lambda_{1}(x), \lambda_{2}(x)$ are the eigenvalues of $(d \varphi(x))^{*} \circ d \varphi(x)$. Notice that given a homeomorphism $\varphi \in \mathcal{H}_{\varepsilon}\left(\mathbf{S}, \mathbf{S}^{\prime}\right)$ the image $C^{\varphi}=\varphi(C)$ of a compact component $C$ of $\overline{\operatorname{Thin}_{\varepsilon}(\mathbf{S})} \subset \dot{S}^{D, r}$ is a compact component of $\overline{\operatorname{Thin}_{\varepsilon}\left(\mathbf{S}^{\prime}\right)} \subset\left(\dot{S}^{\prime}\right)^{D^{\prime}, r^{\prime}}$. Let us consider the components $\widehat{C}$ of $\overline{\operatorname{Thin}_{\varepsilon_{0} / 2}(\mathbf{S})} \subset \dot{S}^{D, r}$ and and $\widehat{C}^{\prime}$ of $\overline{\operatorname{Thin}_{\varepsilon_{0} / 2}\left(\mathbf{S}^{\prime}\right)} \subset$ $\left(\dot{S}^{\prime}\right)^{D^{\prime}, r^{\prime}}$ which contain $C$ and $C^{\varphi}$, respectively. Let $S_{ \pm} S_{ \pm}^{\prime}$ be the boundary circles of $\widehat{C}$ and $\widehat{C}^{\prime}$, and $\Gamma, \Gamma^{\prime}$ be their central geodesics or special curves. Let $\pi: C \rightarrow \Gamma$ and $\pi^{\prime}: C^{\prime} \rightarrow \Gamma^{\prime}$ be the projections along the geodesics orthogonal to $\Gamma$ and $\Gamma^{\prime}$. For any point $x \in \Gamma$ take the points $x_{ \pm} \in S_{ \pm}$such that $\pi\left(x_{ \pm}\right)=x$. Similarly, we define point $x_{ \pm}^{\prime} \in S_{ \pm}^{\prime}$ for any point $x^{\prime} \in \Gamma^{\prime}$. Let $\delta(x)$ denote the distance between the points $\pi^{\prime}\left(\varphi\left(x_{+}\right)\right)$and $\pi^{\prime}\left(\varphi\left(x_{-}\right)\right)$in $\Gamma^{\prime}$ measured with respect to the arc length metric on $\Gamma^{\prime}$. Similarly, $\delta^{\prime}\left(x^{\prime}\right)$ denote the distance between the points $\pi\left(\varphi^{-1}\left(x_{+}^{\prime}\right)\right)$ and $\pi\left(\varphi^{-1}\left(x_{-}^{\prime}\right)\right)$ in $\Gamma$ measured with respect to the arc length metric on $\Gamma$. We assume here that the total length of $\Gamma$ and $\Gamma^{\prime}$ is normalized by 1 . Next, set

$$
\delta_{C}(\varphi)=\sup _{x \in \Gamma}(\delta(x))+\sup _{x^{\prime} \in \Gamma}\left(\delta\left(x^{\prime}\right)\right) .
$$

Clearly, $\delta_{C}(\varphi)$ is independent of the ambiguity in choosing the ordering of the boundary circles of $\widehat{C}$ and $\widehat{C}^{\prime}$. We set

$$
d_{\varepsilon}^{\varphi}\left((\mathbf{S}, r),\left(\mathbf{S}^{\prime}, r^{\prime}\right)\right)=K_{\varepsilon}(\varphi)+\sum_{C} \delta_{C}(\varphi),
$$

where the sum is taken over all compact components $C$ of $\overline{\operatorname{Thin}}_{\varepsilon}(\mathbf{S}) \subset \dot{S}^{D, r}$, and

$$
d_{\varepsilon}\left((\mathbf{S}, r),\left(\mathbf{S}^{\prime}, r^{\prime}\right)\right)=\min \left\{1, \inf _{\varphi \in \mathcal{H}_{\varepsilon}\left(\mathbf{S}, \mathbf{S}^{\prime}\right)} d_{\varepsilon}^{\varphi}\left((\mathbf{S}, r),\left(\mathbf{S}^{\prime}, r^{\prime}\right)\right)\right\}
$$

Next, we define the distance function $d\left((\mathbf{S}, r),\left(\mathbf{S}^{\prime}, r^{\prime}\right)\right)$ by the formula

$$
d\left((\mathbf{S}, r),\left(\mathbf{S}^{\prime}, r^{\prime}\right)\right)=\sum_{i=1}^{\infty} \frac{d_{1 / 2^{i}}\left((\mathbf{S}, r),\left(\mathbf{S}^{\prime}, r^{\prime}\right)\right)}{2^{i}} .
$$

Proposition B.1 Formula (53) defines a metric on $\overline{\mathcal{M}}_{g, \mu}^{\$}$.

Proof The only non-obvious properties which we need to check is the triangle inequality and the non-degeneracy of the distance function $d$.

Triangle inequality Let us verify the triangle inequality for $d_{\varepsilon}$. The inequality for $d$ then follows. Suppose that we are given three stable nodal Riemann surfaces $\left(\mathbf{S}_{1}, r_{1}\right),\left(\mathbf{S}_{2}, r_{2}\right),\left(\mathbf{S}_{3}, r_{3}\right)$. Denote $\mathcal{H}_{i j}=\mathcal{H}_{\varepsilon}\left(\mathbf{S}_{i}, \mathbf{S}_{j}\right)$. If 
$\mathcal{H}_{12}=\mathcal{H}_{13}=\mathcal{H}_{23}=\varnothing$, or if $\mathcal{H}_{12}=\mathcal{H}_{13}=\varnothing$ but $\mathcal{H}_{23} \neq \varnothing$ then the triangle inequality is obviously satisfied. If $\varphi_{12} \in \mathcal{H}_{12}$ and $\varphi_{23} \in \mathcal{H}_{23}$ then $\varphi_{13}=\varphi_{1} \circ \varphi_{2} \in \mathcal{H}_{13}$. Notice that

$$
K_{\varepsilon}\left(\varphi_{13}\right) \leq K_{\varepsilon}\left(\varphi_{12}\right)+K_{\varepsilon}\left(\varphi_{23}\right)
$$

and, for every component $C$ of $\operatorname{Thin}_{\varepsilon}\left(\mathbf{S}_{1}\right) \subset S^{D_{1}, r_{1}}$,

$$
\delta_{C}\left(\varphi_{13}\right) \leq \delta_{C}\left(\varphi_{12}\right)+\delta_{\varphi_{12}(C)}\left(\varphi_{23}\right) .
$$

Hence,

$$
\begin{aligned}
d_{\varepsilon}^{\varphi_{13}}\left(\left(\mathbf{S}_{1}, r_{1}\right),\left(\mathbf{S}_{3}, r_{3}\right)\right) & \leq K_{\varepsilon}\left(\varphi_{13}\right)+\sum_{C} \delta_{C}\left(\varphi_{13}\right) \\
& \leq K_{\varepsilon}\left(\varphi_{12}\right)+\sum_{C} \delta_{C}\left(\varphi_{12}\right) \\
& +K_{\varepsilon}\left(\varphi_{23}\right)+\sum_{C^{\prime}} \delta_{C^{\prime}}\left(\varphi_{23}\right) \\
& =d_{\varepsilon}^{\varphi_{12}}\left(\left(\mathbf{S}_{1}, r_{1}\right),\left(\mathbf{S}_{2}, r_{2}\right)\right)+d_{\varepsilon}^{\varphi_{23}}\left(\left(\mathbf{S}_{2}, r_{2}\right),\left(\mathbf{S}_{3}, r_{3}\right)\right)
\end{aligned}
$$

where the first two sums are taken over all compact components

$$
C \subset \operatorname{Thin}_{\varepsilon}\left(\mathbf{S}_{1}\right) \subset \dot{S}^{D_{1}, r_{1}},
$$

and the third sum is taken over all compact components

$$
C^{\prime} \subset \operatorname{Thin}_{\varepsilon}\left(\mathbf{S}_{2}\right) \subset \dot{S}^{D_{2}, r_{2}} .
$$

Hence

$$
d_{\varepsilon}\left(\left(\mathbf{S}_{1}, r_{1}\right),\left(\mathbf{S}_{3}, r_{3}\right)\right) \leq d_{\varepsilon}\left(\left(\mathbf{S}_{1}, r_{1}\right),\left(\mathbf{S}_{2}, r_{2}\right)\right)+d_{\varepsilon}\left(\left(\mathbf{S}_{2}, r_{2}\right),\left(\mathbf{S}_{3}, r_{3}\right)\right) .
$$

Non-degeneracy Suppose that

$$
d\left((\mathbf{S}, r),\left(\mathbf{S}^{\prime}, r^{\prime}\right)\right)=0 .
$$

Then $d_{1 / 2^{i}}\left((\mathbf{S}, r),\left(\mathbf{S}^{\prime}, r^{\prime}\right)\right)=0$ for all $i \geq 0$. Taking into account that the space of quasi-conformal homeomorphisms $\operatorname{Thick}_{\varepsilon}(\mathbf{S}) \rightarrow \operatorname{Thick}_{\varepsilon}\left(\mathbf{S}^{\prime}\right)$ of bounded distortion is compact for every $\varepsilon>0$ and by passing to a diagonal subsequence we conclude that there exists a sequence of homeomorphisms $\varphi_{k} \in \mathcal{H}_{1 / 2^{k}}\left(\mathbf{S}, \mathbf{S}^{\prime}\right)$, for $k \geq 1$, which converges uniformly on the thick parts to a quasi-conformal homeomorphism of zero conformal distortion, and hence to a biholomorphism

$$
\varphi:\left(\dot{S}^{D, r} \backslash \bigcup \Gamma_{i}, j\right) \rightarrow\left(\left(\dot{S}^{\prime}\right)^{D, r} \backslash \bigcup \Gamma_{i}^{\prime}, j^{\prime}\right) .
$$

The removal of singularities now allows us to extend the biholomorphism $\varphi$ to an equivalence between the nodal Riemann surfaces $\mathbf{S}=(S, j, M, D)$ and $\mathbf{S}^{\prime}=\left(S^{\prime}, j^{\prime}, M^{\prime}, D^{\prime}\right)$. On the other hand, we also have for any special circle $\Gamma_{i}$

$$
\delta_{C_{i}}\left(\varphi_{k}\right) \underset{k \rightarrow \infty}{\longrightarrow} 0,
$$


where $C_{i}$ is the component of $\overline{\operatorname{Thin}_{1 / 2^{k}}(\mathbf{S})} \subset \dot{S}^{D, r}$ which contains $\Gamma_{i}$. But that implies that $\varphi$ is an equivalence of the decorated nodal Riemann surfaces $(\mathbf{S}, r)$ and $\left(\mathbf{S}^{\prime}, r^{\prime}\right)$.

The metric on $\overline{\mathcal{M}}_{g, \mu}$ can be defined by a formula similar to (51)-(53) but without the term $\sum_{C} \delta_{C}(\varphi)$. In other words, the metric on $\overline{\mathcal{M}}_{g, \mu}$ is by definition the push-forward of the metric on $\overline{\mathcal{M}}_{g, \mu}^{\$}$ under the canonical projection $\overline{\mathcal{M}}_{g, \mu}^{\$} \rightarrow$ $\overline{\mathcal{M}}_{g, \mu}$.

\section{B.2 Metric on $\overline{\mathcal{M}}_{g, \mu, p_{-}, p_{+}}(V)$}

We will define a metric on $\overline{\mathcal{M}}_{g, \mu, p_{-}, p_{+}}(V)$ similar to the way it was done in Appendix B.1 above for the Deligne-Mumford compactification of the space of Riemann surfaces. Take (the equivalency classes of) two stable holomorphic building $(F, \Phi)$ and $\left(F^{\prime}, \Phi^{\prime}\right)$ of the same signature $\left(g, \mu, p^{+}, p^{-}\right)$, and of height $k$ and $k^{\prime} \leq k$, respectively. Let

$$
\left(\mathbf{S}=\mathbf{S}_{F}, r=r_{F, \Phi}\right) \quad \text { and } \quad\left(\mathbf{S}^{\prime}=\mathbf{S}_{F^{\prime}}, r^{\prime}=r_{F^{\prime}, \Phi^{\prime}}\right)
$$

be the underlying decorated Riemann nodal surfaces. Suppose first that the decorated Riemann surfaces

$$
\mathbf{S}=(S, M, D, r) \quad \text { and } \quad \mathbf{S}^{\prime}=\left(S^{\prime}, M^{\prime}, D^{\prime}, r^{\prime}\right)
$$

are stable and consider their deformations $S^{D, r}$ and $\left(S^{\prime}\right)^{D^{\prime}, r^{\prime}}$, as in Section 4.4 and Appendix B.1 above. The surfaces $\dot{S}^{D, r}=S^{D, r} \backslash M$ and $\left(\dot{S}^{\prime}\right)^{D^{\prime}, r^{\prime}}=$ $\left(S^{\prime}\right)^{D^{\prime}, r^{\prime}} \backslash M^{\prime}$ are endowed with the uniformizing hyperbolic metrics, which are degenerate along the special circles corresponding to the double points. Fix an $\varepsilon<\varepsilon_{0}$ and consider the $\varepsilon$-thick parts $\operatorname{Thick}_{\varepsilon}(\mathbf{S}) \subset \dot{S}^{D, r}$ and $\operatorname{Thick}_{\varepsilon}\left(\mathbf{S}^{\prime}\right) \subset$ $\left(\dot{S}^{\prime}\right)^{D^{\prime}, r^{\prime}}$. Let the notation $\mathcal{H}_{\varepsilon}\left(\mathbf{S}, \mathbf{S}^{\prime}\right)$ and $d_{\varepsilon}^{\varphi}\left((\mathbf{S}, r),\left(\mathbf{S}^{\prime}, r^{\prime}\right)\right)$ for $\varphi \in \mathcal{H}_{\varepsilon}\left(\mathbf{S}, \mathbf{S}^{\prime}\right)$ have the same meaning as in Appendix B.1. Let us recall that the $V$-components $f$ and $f^{\prime}$ of the maps $F$ and $F^{\prime}$ continuously extend to the maps $\bar{f}: \bar{S} \rightarrow V$ and $\bar{f}^{\prime}: \bar{S}^{\prime} \rightarrow V$, where $\bar{S}$ and $\bar{S}^{\prime}$ are oriented blow-ups of the surfaces $S^{D, r}$ and $\left(S^{\prime}\right)^{D^{\prime}, r^{\prime}}$ along the sets $M$ and $M^{\prime}$ of marked points, as it was described in Section 4.3 above. Let us set

$$
\tilde{d}_{\varepsilon}^{\varphi}\left((F, \Phi),\left(F^{\prime}, \Phi^{\prime}\right)\right)=\rho\left(\bar{f}, \bar{f}^{\prime} \circ \varphi\right),
$$

where $\rho$ is the $C^{0}$-distance between mappings of a compact surface $\bar{S}$ into the manifold $V$ endowed with a Riemannian metric. Next, we define the $\varepsilon$-level of a connected component of $\operatorname{Thick}_{\varepsilon}(\mathbf{S})$. Given two components $C, C^{\prime} \subset \operatorname{Thick}_{\varepsilon}(\mathbf{S})$, we say that $C^{\prime}$ has a bigger $\varepsilon$-level than $C$, and write $C \prec_{\varepsilon} C^{\prime}$ if either 


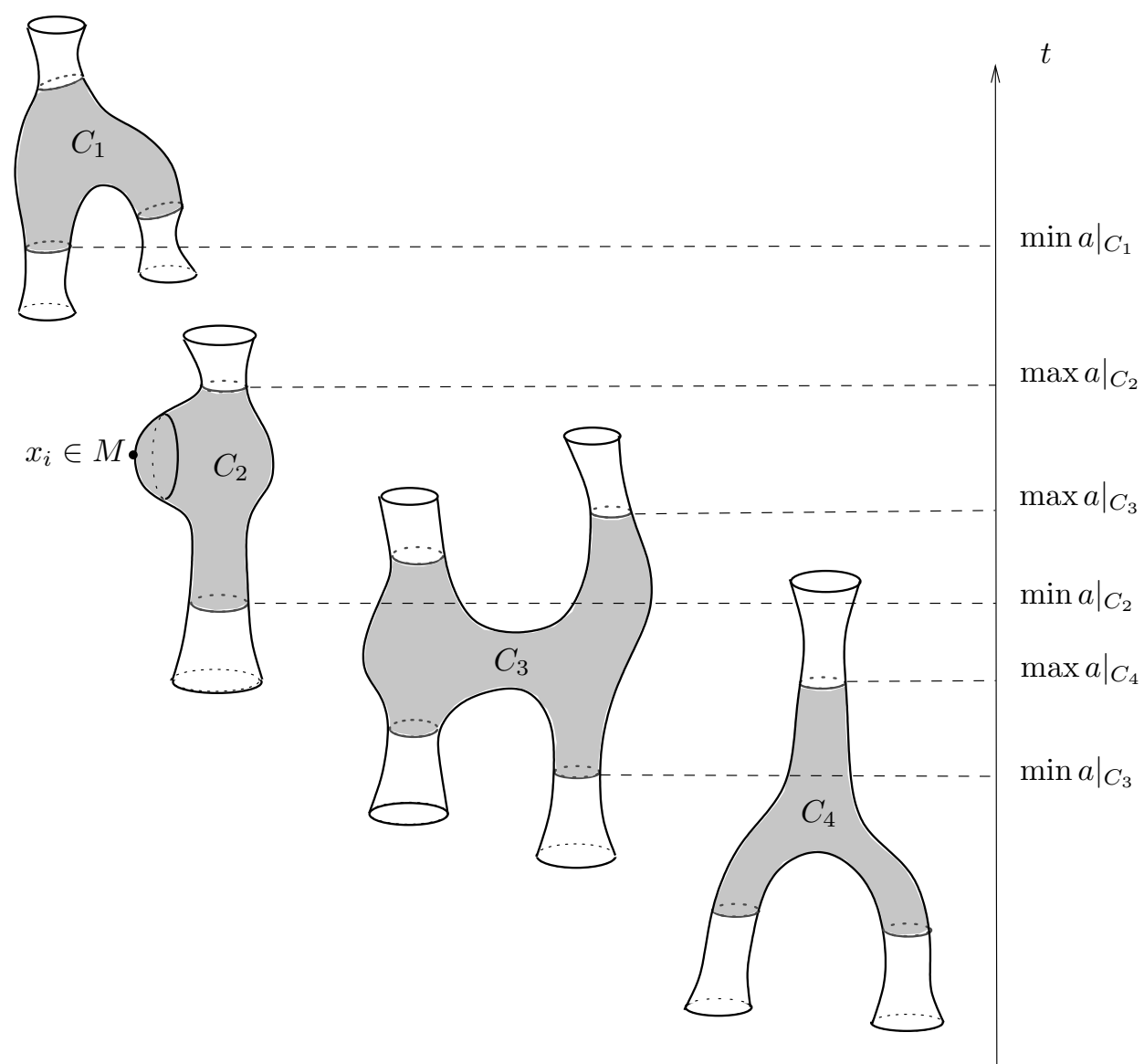

Figure 16: $C_{2}, C_{3}, C_{4} \prec_{\varepsilon} C_{1} ; C_{2}, C_{3}, C_{4}$ are on the same $\varepsilon$-level

- the level of $C$ is less than the level of $C^{\prime}$ in the building $(F, \Phi)$, or

- $C$ and $C^{\prime}$ belong to the same level of the building $(F, \Phi)$, and

$$
\left.\min a\right|_{C^{\prime}}>\left.\max a\right|_{C},
$$

and one cannot find a sequence of components

$$
C_{1}=C, C_{2}, \ldots, C_{k}=C^{\prime}
$$

which belong to the same level of the building $(F, \Phi)$ and satisfy

$$
\left.\max a\right|_{C_{i}} \geq\left.\max a\right|_{C_{i-1}} \text { but } \min a_{C_{i}} \leq\left.\max a\right|_{C_{i-1}} \text { for } i=2, \ldots, k \text {. }
$$

We define the $\varepsilon$-level of the component $C \subset \operatorname{Thick}_{\varepsilon}(\mathbf{S})$ as $\max \left\{k \mid\right.$ there exist $\left.C_{1}, \ldots, C_{k-1} \subset \operatorname{Thick}_{\varepsilon}(\mathbf{S}), C_{1} \prec_{\varepsilon} \cdots \prec_{\varepsilon} C_{k-1} \prec_{\varepsilon} C\right\}$. 
Similarly we define the $\varepsilon$-level of components of $\operatorname{Thick}_{\varepsilon}\left(\mathbf{S}^{\prime}\right)$. Let us denote by $C_{i}^{\varepsilon}$, for $i=1, \ldots, k$, the union of components of $\operatorname{Thick}_{\varepsilon}(\mathbf{S})$ of $\varepsilon$-level $i$. We set

$$
\hat{d}_{\varepsilon}^{\varphi}\left(F, F^{\prime}\right)=1
$$

if there exists at least one component $C \subset \operatorname{Thick}_{\varepsilon}(\mathbf{S})$ which has a different $\varepsilon$-level than the component $\varphi(C) \subset \operatorname{Thick}_{\varepsilon}\left(\mathbf{S}^{\prime}\right)$. Otherwise we set

$$
\hat{d}_{\varepsilon}^{\varphi}\left(F, F^{\prime}\right)=\min \left\{1, \sum_{1}^{k} \inf _{c \in \mathbb{R}}\left\|c+a_{i}-a_{i}^{\prime} \circ \varphi\right\|_{C^{0}\left(C_{i}^{\varepsilon}\right)}\right\},
$$

and define

$$
\begin{aligned}
& D_{\varepsilon}\left((F, \Phi),\left(F^{\prime}, \Phi^{\prime}\right)\right) \\
& =\min \left(1, \inf _{\varphi \in \mathcal{H}_{\varepsilon}\left(\mathbf{S}, \mathbf{S}^{\prime}\right)}\left(d_{\varepsilon}^{\varphi}\left((\mathbf{S}, r),\left(\mathbf{S}^{\prime}, r^{\prime}\right)\right)+\tilde{d}_{\varepsilon}^{\varphi}\left((F, \Phi),\left(F^{\prime}, \Phi^{\prime}\right)\right)+\hat{d}_{\varepsilon}^{\varphi}\left(F, F^{\prime}\right)\right)\right) .
\end{aligned}
$$

Next, we introduce a distance function $D\left((F, \Phi),\left(F^{\prime}, \Phi^{\prime}\right)\right)$ by the formula

$$
D\left((F, \Phi),\left(F^{\prime}, \Phi^{\prime}\right)\right)=\sum_{1}^{\infty} \frac{1}{2^{j}} D_{1 / 2^{j}}\left((F, \Phi),\left(F^{\prime}, \Phi^{\prime}\right)\right) .
$$

Let us denote by $U_{0}$ the subset of curves from $\overline{\mathcal{M}}_{g, \mu, p_{-}, p_{+}}(V)$ for which the underlying Riemann surfaces are stable. It is now straightforward to verify the following proposition.

Proposition B.2 The distance function $D$ is a metric on $U_{0}$.

If the surfaces $\mathbf{S}$ and/or $\mathbf{S}^{\prime}$ are unstable we first add extra sets $L$ and $L^{\prime}$, $\# L=\# L^{\prime}=l$, of marked points to each of the holomorphic buildings to stabilize their underlying surfaces. Let us denote by $U_{l}$ the subset of buildings from $\overline{\mathcal{M}}_{g, \mu, p_{-}, p_{+}}(V)$ which can be stabilized by adding $\leq l$ marked points, and define for two curves $(F, \Phi),\left(F^{\prime}, \Phi^{\prime}\right) \in U_{l} \subset \overline{\mathcal{M}}_{g, \mu, p_{-}, p_{+}}(V)$ the distance $D^{l}\left((F, \Phi),\left(F^{\prime}, \Phi^{\prime}\right)\right)$ by the formula

$$
D^{l}\left((F, \Phi),\left(F^{\prime}, \Phi^{\prime}\right)\right)=\min \left\{1, \inf _{\substack{L, L^{\prime} \\ \# L=\# L^{\prime}=l}} d\left(F^{L},\left(F^{\prime}\right)^{L^{\prime}}\right)\right\},
$$

where the infimum is taken over all sets $L$ and $L^{\prime}$ of cardinality $l$ which stabilize the surfaces $\mathbf{S}$ and $\mathbf{S}^{\prime}$.

Proposition B.3 $D^{l}$ is a metric on $U_{l}$. 
Proof We only need to verify the non-degeneracy of $D^{l}$. Suppose that

$$
D^{l}\left((F, \Phi),\left(F^{\prime}, \Phi^{\prime}\right)\right) \rightarrow 0 .
$$

Then there exist sequences of extra sets of marked points $L_{k}$ on $S$ and $L_{k}^{\prime}$ on $S^{\prime}, k=1, \ldots$, such that $D\left(\left(F^{L_{k}}, \Phi\right),\left(\left(F^{\prime}\right)^{L_{k}^{\prime}}, \Phi^{\prime}\right)\right)=0$. In view of the compactness of the moduli space of stable nodal Riemann surfaces (see Theorem 4.2) we can assume, after possibly passing to a subsequence, that

$$
\left(F^{L_{j}}, \Phi\right) \rightarrow\left(F^{\bar{L}}, \Phi\right)
$$

and

$$
\left(\left(F^{\prime}\right)^{L_{j}^{\prime}}, \Phi^{\prime}\right) \rightarrow\left(\left(F^{\prime}\right)^{L^{\prime}}, \Phi^{\prime}\right) .
$$

The stable Riemann surfaces $\overline{\mathbf{S}}$ and $\overline{\mathbf{S}}^{\prime}$ which underly the holomorphic buildings $\left(F^{\bar{L}}, \Phi\right)$ and $\left(\left(F^{\prime}\right)^{\bar{L}^{\prime}}, \Phi^{\prime}\right)$ are limits of sequences of stable Riemann surfaces $\mathbf{S}_{k}$ and $\mathbf{S}_{k}^{\prime}$, for $k \geq 1$, underlying $F^{L_{k}}$ and $\left(F^{\prime}\right)^{L_{k}^{\prime}}$. We have

$$
D\left(\left(F^{\bar{L}}, \Phi\right),\left(\left(F^{\prime}\right)^{\bar{L}^{\prime}}, \Phi\right)\right)=0
$$

and therefore can apply Proposition B.2 to finish the proof.

Next, we extend the distance function $D^{l}$ to $\overline{\mathcal{M}}_{g, \mu, p_{-}, p_{+}}(V)$ by setting

$$
D^{l}\left((F, \Phi),\left(F^{\prime}, \Phi^{\prime}\right)\right)= \begin{cases}0, & \text { if both } \left.(F, \Phi) \text { and } F^{\prime}, \Phi^{\prime}\right) \text { are not in } U_{l} ; \\ 1, & \text { if one of the curves is in } U_{l} \text { and the other one } \\ & \text { is not. }\end{cases}
$$

Of course, the distance function $D^{l}$ extended this way to the whole moduli space $\overline{\mathcal{M}}_{g, \mu, p_{-}, p_{+}}(V)$ is degenerate, and hence is not a metric. However, it is still a pseudo-metric, and in particular satisfies the triangle inequality. Let us also note that $D^{k} \leq D^{l}$ for $k \geq l$. Finally we define the required metric $D^{\text {stable }}$ on $\overline{\mathcal{M}}_{g, \mu, p_{-}, p_{+}}(V)$ by the formula

$$
D^{\text {stable }}=\sum_{1}^{\infty} \frac{D^{l}}{2^{l}}
$$

Proposition B.4 The distance function $D^{\text {stable }}$ is a metric on $\overline{\mathcal{M}}_{g, \mu, p_{-}, p_{+}}(V)$.

Proof The only thing to check is that $D^{\text {stable }}$ is non-degenerate. Suppose $D^{\text {stable }}\left((F, \Phi),\left(F^{\prime}, \Phi^{\prime}\right)\right)=0$. Then $D^{l}\left((F, \Phi),\left(F^{\prime}, \Phi^{\prime}\right)\right)=0$ for all $l \geq 0$. Suppose that both buildings $(F, \Phi)$ and $\left(F^{\prime}, \Phi^{\prime}\right)$ can be stabilized by sets of cardinality $k$. Then it follows from Proposition B.3 that $D^{k}\left((F, \Phi),\left(F^{\prime}, \Phi^{\prime}\right)\right)=$ 0 implies $(F, \Phi)=\left(F^{\prime}, \Phi^{\prime}\right)$. 


\section{References}

[1] F Bourgeois, A Morse-Bott approach to Contact Homology, PhD Dissertation, Stanford University (2002)

[2] P Deligne, D Mumford, Irrecducibility of the space of curves of give genus, Publ. Math. IHES, 36 (1969) 75-109

[3] T Ekholm, J Etnyre, M Sullivan, Legendrian Submanifolds in $\mathbb{R}^{2 n+1}$ and Contact Homology, arXiv:math.SG/0210124

[4] Y Eliashberg, A Givental, H Hofer, Introduction to Symplectic Field Theory Geom. Funct. Anal. Special Volume, Part II (2000) 560-673

[5] Y Eliashberg, H Hofer, D Salamon, Lagrangian intersections in contact geometry, Geom. and Funct. Anal. 5 (1995) 244-269

[6] A Floer, The unregularized gradient flow of the symplectic action, Comm. Pure Appl. Math., 41 (1988) 775-813

[7] A Floer, Morse theory for Lagrangian intersections, J. Differential Geom. 28 (1988) 513-547

[8] M Gromov, Pseudo-holomorphic curves in symplectic manifolds, Invent. Math. 82 (1985) 307-347

[9] H Hofer, Pseudo-holomorphic curves and Weinstein conjecture in dimension three, Invent. Math. 114 (1993) 307-347

[10] H Hofer, C Viterbo, The Weinstein conjecture in the presence of holomorphic spheres, Comm. Pure Appl. Math. 45 (1992) 583-622

[11] H Hofer, E Zehnder, Symplectic Invariants and Hamiltonian Dynamics, Birkhäuser Advanced Texts, 1994

[12] H Hofer, K Wysocki, E Zehnder, Pseudoholomorphic curves in symplectizations I: Asymptotics, Ann. Inst. Henri Poincaré, Analyse Nonlinéaire 13 (1996) No. 3, 337-379

[13] H Hofer, K Wysocki, E Zehnder, Properties of Pseudoholomorphic Curves in Symplectization IV. Asymptotics with degeneracies, from: "Contact and Symplectic Geometry", Cambridge University Press (1996) 78-117

[14] H Hofer, K Wysocki, E Zehnder, Correction to "Properties of Pseudoholomorphic Curves in Symplectization I, Asymptotics", Ann. Inst. Henri Poincaré, Analyse Nonlinéaire 15 (1998) 535-538

[15] H Hofer, K Wysocki, E Zehnder, The Dynamics on a Strictly Convex Energy Surface in $\mathbb{R}^{4}$, Annals of Mathematics, 148 (1998) 197-289

[16] H Hofer, K Wysocki, E Zehnder, Properties of Pseudoholomorphic Curves in Symplectization III. Fredholm Theory I, from: "Topics in Nonlinear Analysis. The Herbert Amman Anniversary", Birkhäuser Verlag, Basel (1999) 381-475

[17] H Hofer, K Wysocki, E Zehnder, The asymptotic behavior of a finite energy plane, FIM's preprint, ETHZ (2001) 1-35 
[18] H Hofer, K Wysocki, E Zehnder, Finite Energy Cylinders with Small Area, Ergodic Theory Dynam. Systems, 22 (2002) 1451-1486

[19] H Hofer, K Wysocki, E Zehnder, Foliations of the Tight Three Sphere, Annals of Mathematics, 157 (2003) 125-255

[20] C Hummel, Gromov's compactness theorem for pseudo-holomorphic curves, Progress in Mathematics, 151 (1997) Birkhäuser Verlag, Basel

[21] E-N Ionel, T H Parker, Relative Gromov-Witten invariants, Annals of Mathematics, 157 (2003) 45-96

[22] E-N Ionel, T H Parker, The Symplectic Sum Formula for Gromov-Witten Invariants, arXiv:math.SG/0010217

[23] A M Li, Y-B Ruan, Symplectic surgery and Gromov-Witten invariants of Calabi-Yau 3-folds I, Invent. Math. 145 (2001) 151-218

[24] J Li, A degeneration formula of $G W$-invariants, J. Differential Geom. 60 (2002) 199-293

[25] M.-P Muller, Gromov's Schwarz lemma as an estimate of the gradient for holomorphic curves, from: "Holomorphic curves in symplectic geometry", Progr. Math. Birkhaüser, 117 (1994) 217-231

[26] D McDuff, D Salamon, Introduction to Symplectic Topology, Oxford University Press (1998)

[27] D McDuff, D Salamon, J-holomorphic curves and Quantum Cohomology, University Lecture Series 6, AMS (1994)

[28] S A Wolpert, On the Weil-Petersson geometry of the moduli space of curves, Amer. J. Math. 107 (1985) 969-997 